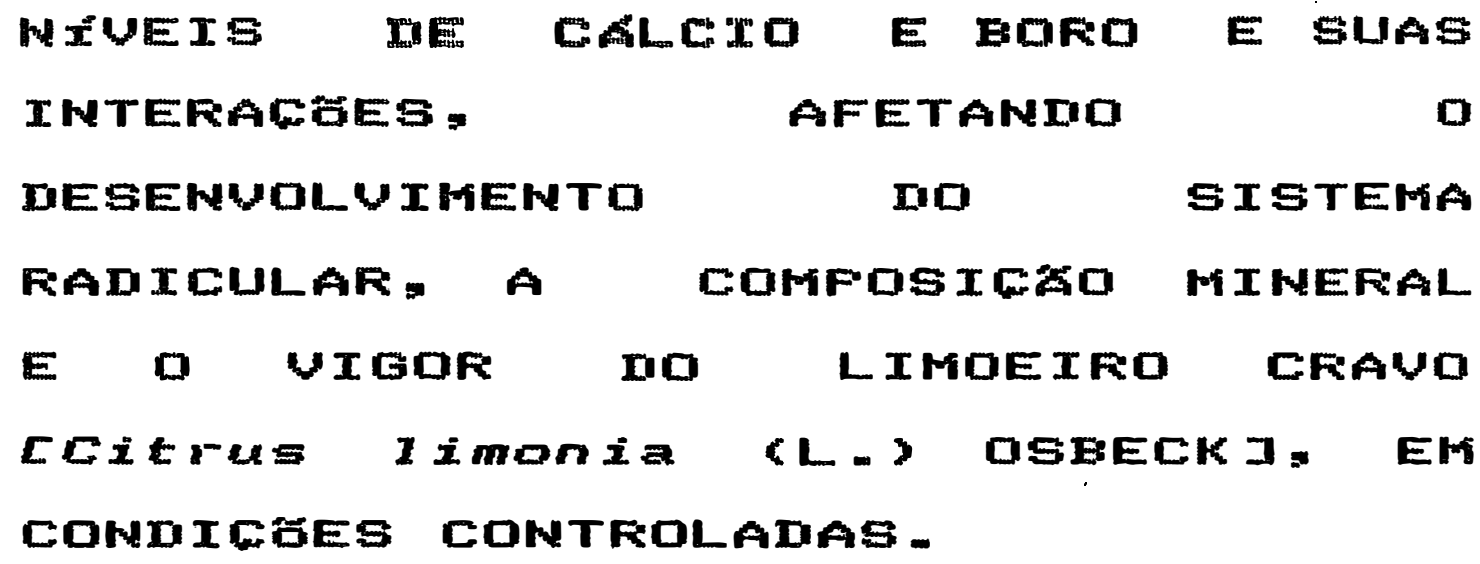

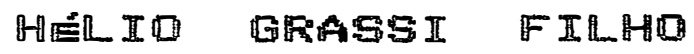

\begin{abstract}
uissertacăo apresentada à Escola Superior de Agricultura "l.uiz de Queiroz" a da Universidade de Sto Faulo. para obtencto do titulo de Mestre riw Agronomia Area de Concentrată Nutrixo de plantas.
\end{abstract}

\author{
$F$ TR A C TCA A A

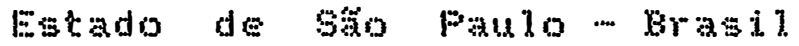 \\ Setcmbro -1991
}


Ficha catalográfica preparada pela Seção de Livros da Divisão de Biblioteca e Documentação - PCAP/USP

Grassi Filho, Hélio

G769n Niveis de cálcio e boro e suas interações, afetando o desenvolvimento do sistema radicular, a composição mi neral e o vigor do limoeiro cravo (Citrus limonia (L.) Osbeck), em condições controladas. Piracicaba, 1991. $92 p$. ilus.

Diss. (Mestre) - ESALQ

Bibliografia.

1. Boro em limão cravo - Efeito 2. Cálcio em limão cravo - Efeito 3. Limão cravo - Porta-enxerto 4. Limão cravo - Nutrição 5. Limão cravo - Raíz - Desenvolvimento I. Escola Superior de Agricultura Luiz de Queiroz, Pira cicaba 


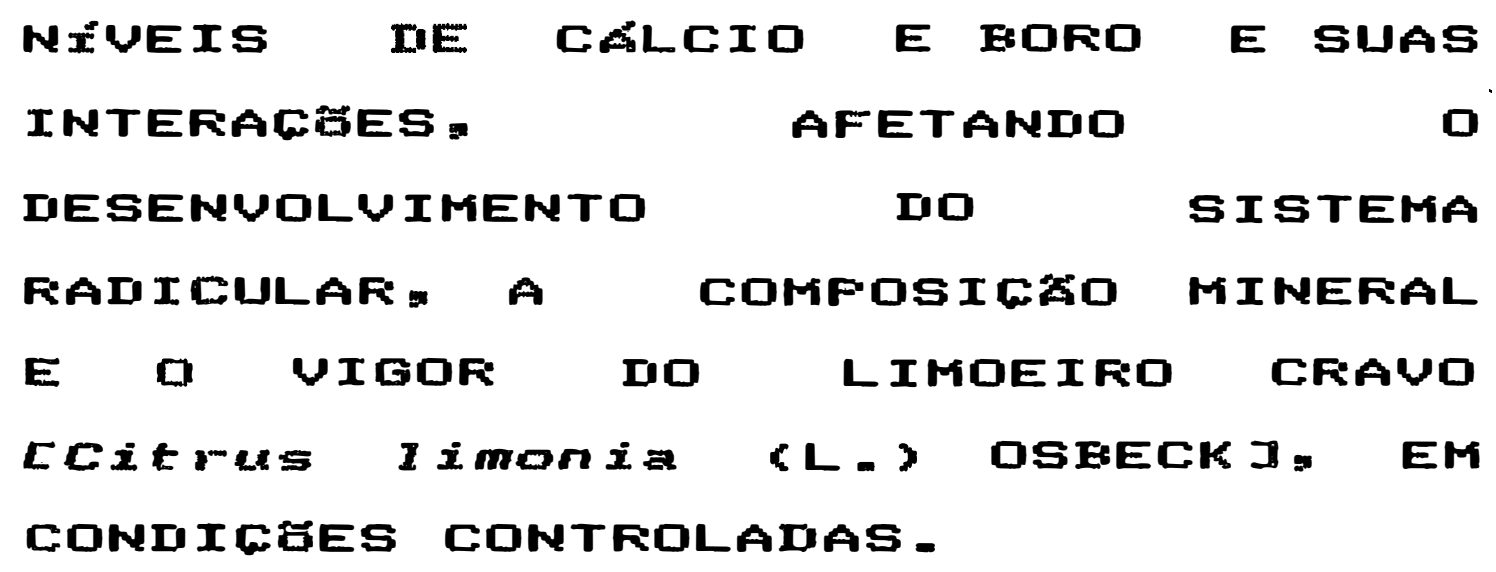

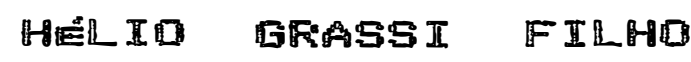

Engenheiro Agrônomo

Orientador: Prof.Dr. ANTONIO ROQUE DECHEN

\begin{abstract}
Missertacto apresentada a Escola Superior de Asricultura "l..uir de Quejroz", da Unjversidade de Săo Fauloy para obtencato do titulo de Mestre Ell Agronomias Area de Concentracto: Solos e Nutricăo de Flantas.
\end{abstract}

\author{
FI II A C I C A \\ Estado de Săo Faulo ... Erasil \\ Setwimo - 1991
}




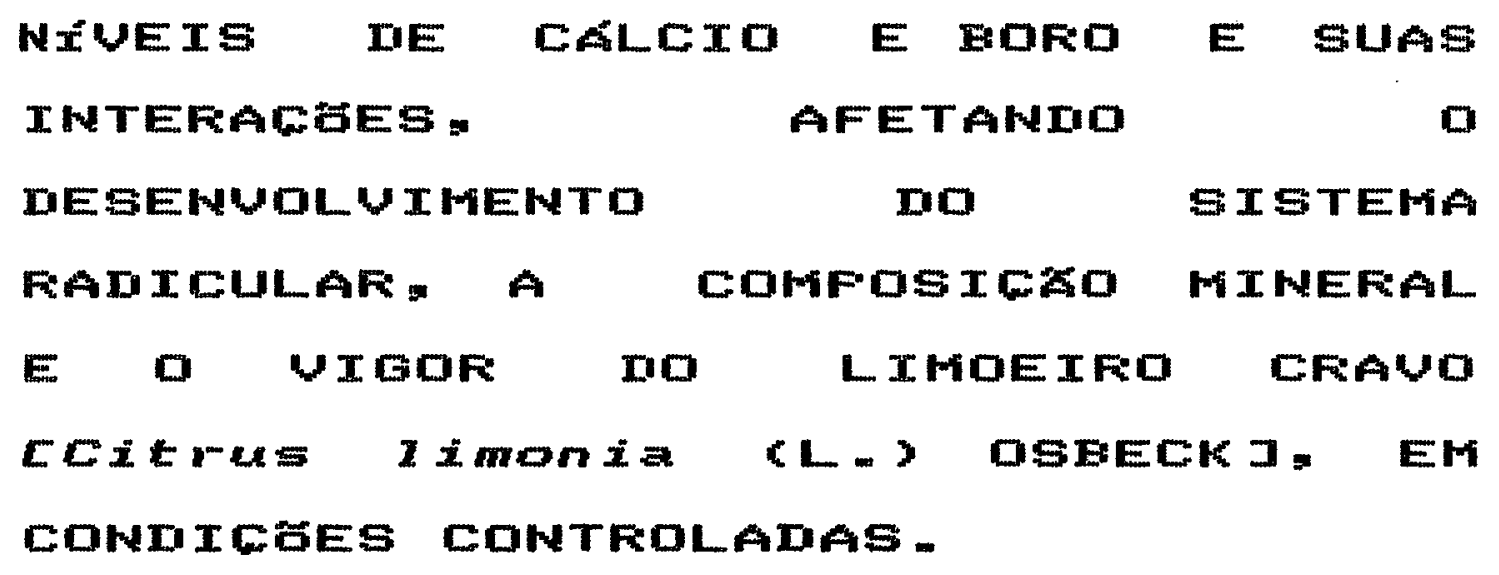

\section{HÉLID GRASSI FILHD}

Aprovada ein: 1.0 .09 .1991

Comissão Julgadora:

Frof. Dr. Antônio Roque Dechen - ESAl_Q/USP

Frof. Mr. Quirino Augusto de Camargo Carme110 - ESALQ/USP

Frof. Mr. Leonardo Theodoro Bull -. FCAMUNESF

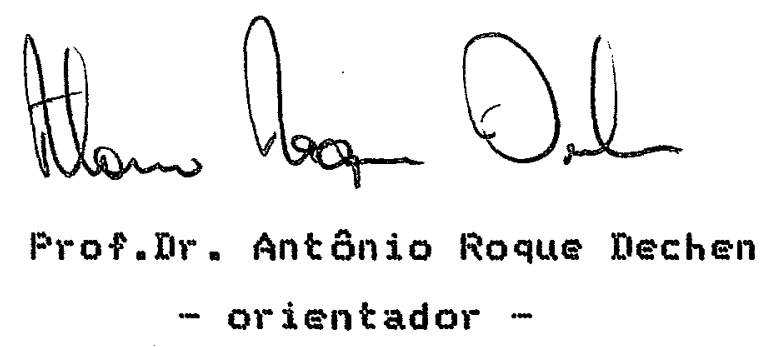


"estas plantinhas săo mudast

- pelo que me disseram, nä.

- e o que fot due elas itsseram?"

"Botânica ao pé da letra"

in: A letra descalca, 1985

Rubens Rodrigues Torres Filho

A meus pais coin
reconhecimento
e gratidão




\section{A G $R A D E L I M E N T D S$}

Ao Frotar. António Foque bechen pela amiagde

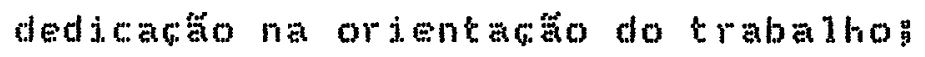

in Frofadra. Leonia Apparecida de l..ima e ao

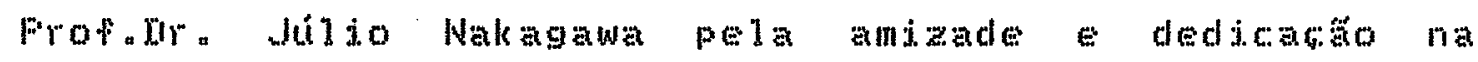
condusto do trabalho:

Ao Liepartamento de Ciencias do solo da Faculdade

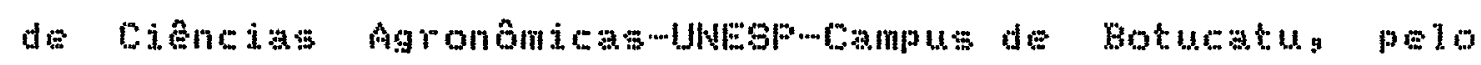
apoio efacildades oferecidaga

Ao Curso de solos e Nutricto de Flantas da

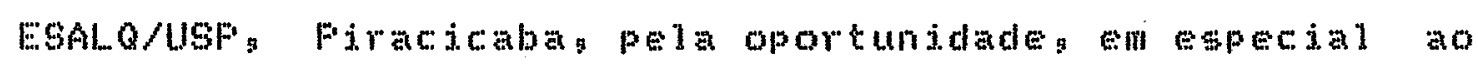
Frofidr "Geraldo victorino de frangag

Ao Sr. José Carlos de Fieri pelo auxilio na condusto do ensaio na casa de vegrtacto

A Srta. Antonja de Fatima Heljodoro pelo auxilio nas medicbes de comprimento de raiz

Ao Sr. Benedito Heliodoro pelo auxilio nas andises quimicas de tecido vegetal

A Suta Eliana Mo Ga Sabino, bibliotecária da

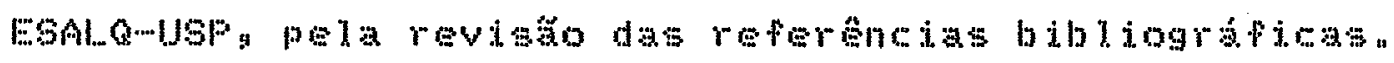

Ao Sra José Garcia Honóro Fires pela digitaca deste trabalho 
Aos funcionários do CINAG a a sr. Roberto carvalho frado pela realianato das andisesestatisticas

A todos que de alguma forma contribuiram para a realizacto deste trabalho. 
SUMARTO

patg.

Resuro a n a

VITI

SUMMARY

$\times]$

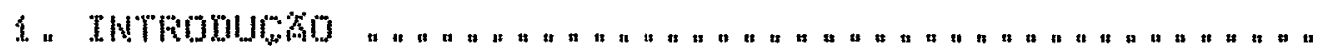

E. REUTSAO DE LITERATURA

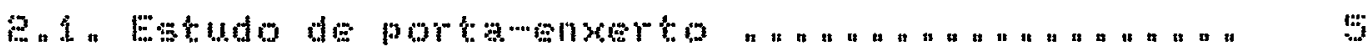

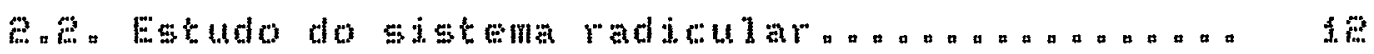

E ne. " Metodos do estudo do sistema radicu

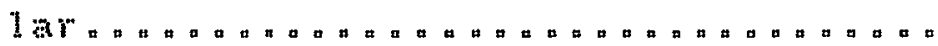

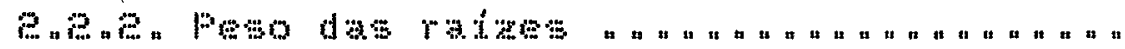

2.3. Estudo do sistema radicular de plantas citrit.

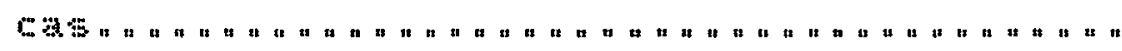

E. 4 a Actimulo de nutrientes

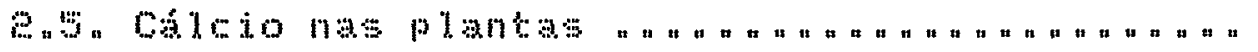

21

2.6. Boro nas plantas "

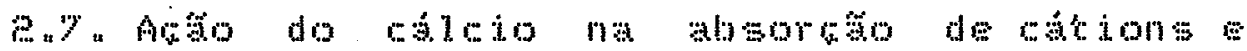
Anjons

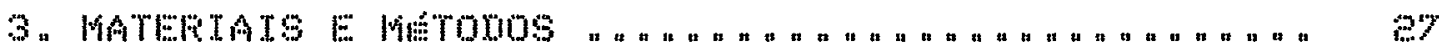

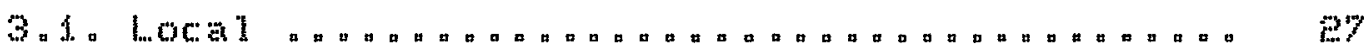

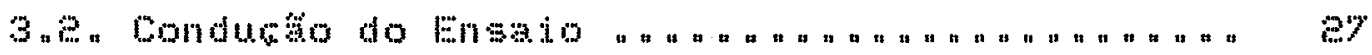

3.

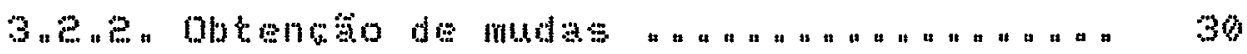

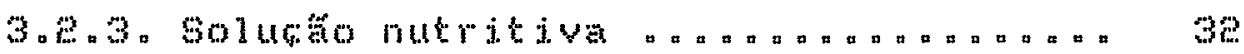

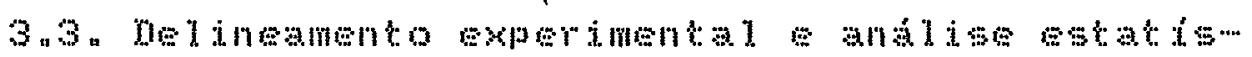


tica 30

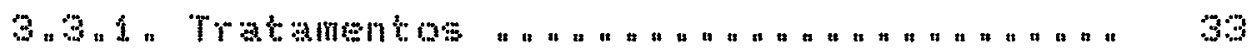

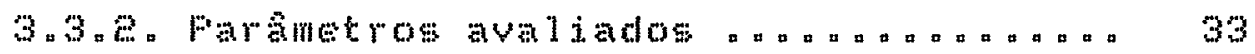

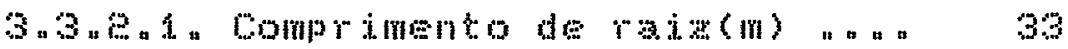

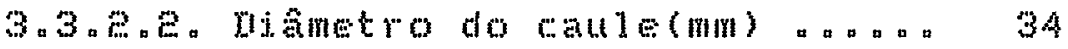

3.3. . . . Ana poliar e numero de fow lhase a a

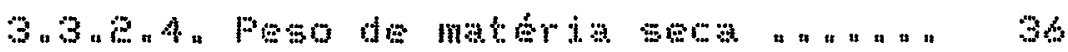

3.3.2.5. Teores de nutrientes "ana: 36

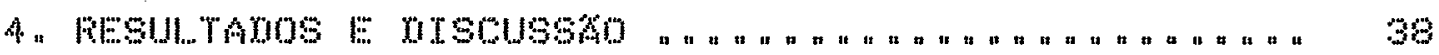

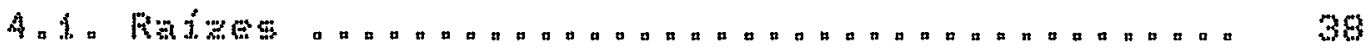

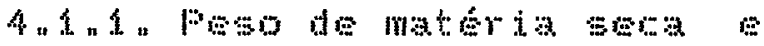

comprimento das ratzes " " " "

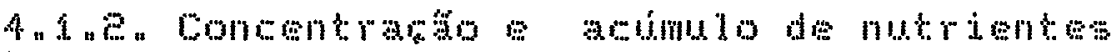

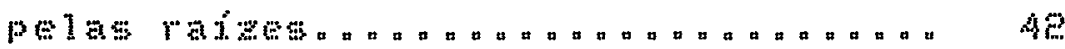

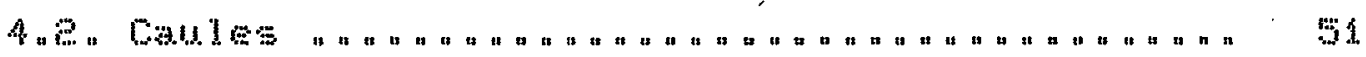

4.e.1. Feso de materia seca

dismetro dos caulest n

51

4.2. a concentracto e actimulo de nutrientes

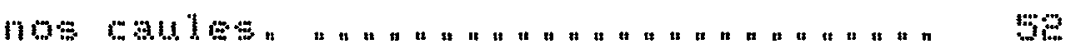

4.3. Folbas a a

$4,3, \ldots+60$ de materia secan

área foljar e numero de folbas a "

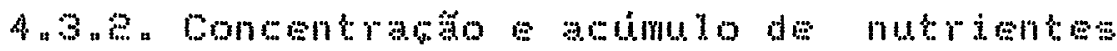
pelas follag

5 CoNCl.

6 BTELTOERAFTA 


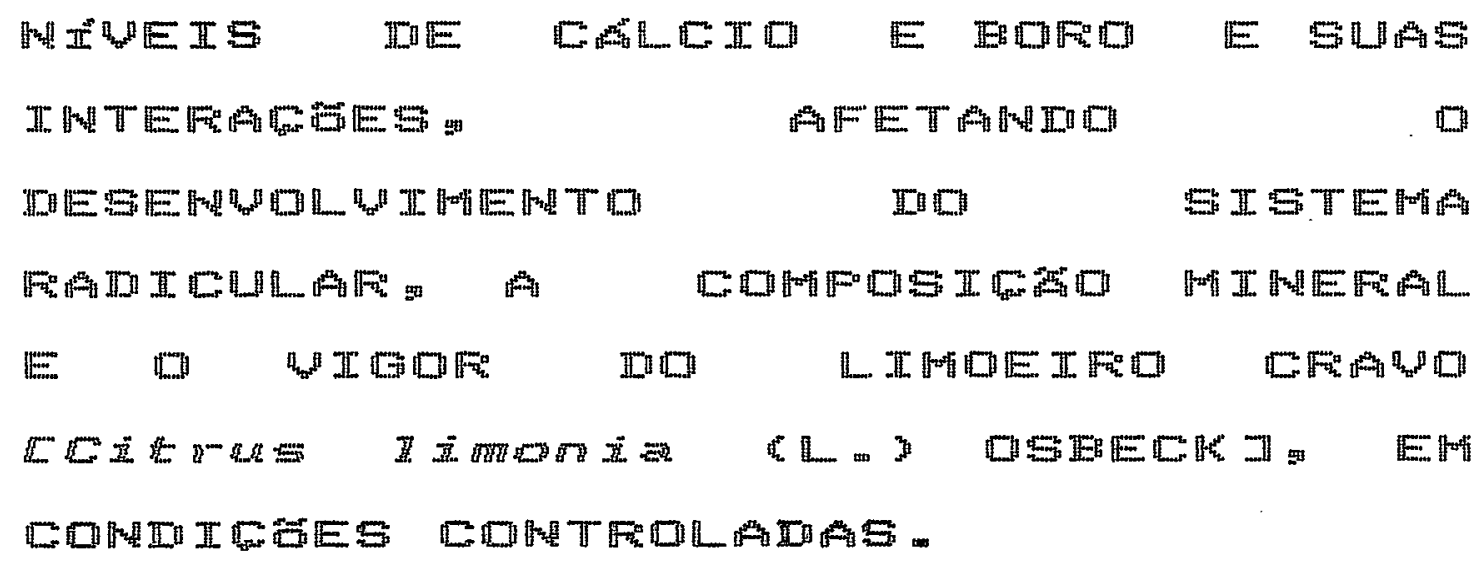

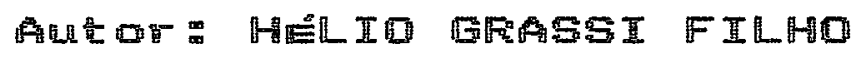

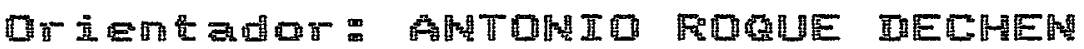

\section{FEESURD}

o presente trabalno teve como objetivon setudar o efeito de niveis de caleion de boro e da interato ca $x$ B no desenvolvimento do sistema radicular. na composiaso mineral we visor do limoetro cravon

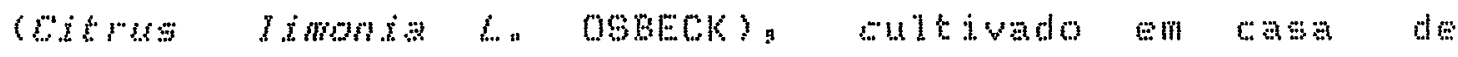
vegetacto.

Fara a realizarto desce estudo utilizoume mudas provenientes de um viveiro existente na razenda Experimental Lageadon as quais foram cultivadas en solurbes mutrivas, com varias concentrabes de calcio 
(50, 100,200 : $400 \mathrm{ppm})$ a de boro $(0,0605,0,125,0,25 \%$

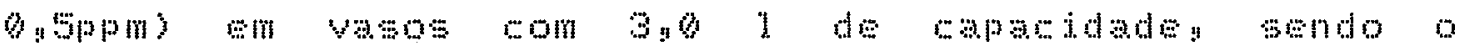
experimento instalado na casa de vegetata do pepartamento

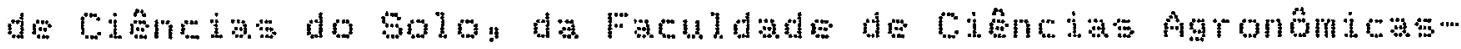
UNESF, Campus de Botucatun o delineanento experinental foi o de blocos casunisados, com lo tratamontos em esquemat fatomal e 4 repetiobes.

A parte experimental teve a duratio de

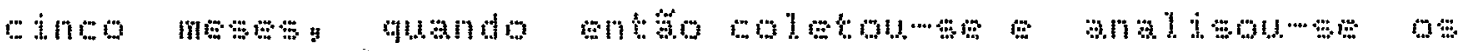
seguintes paranctrosa o peso de materia seca das raizes,

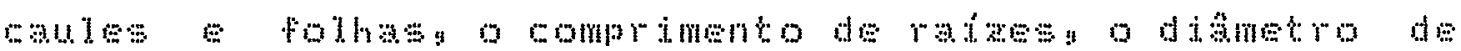

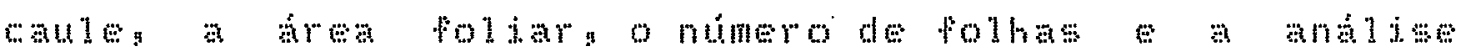
quimica do materal venotal condo sido determinados os nutrientes" nitrogeniog posforos potsssiog cálcion

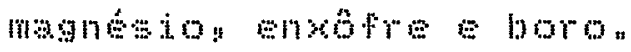

A dose de boro o. 0 mor comprimento de raizes o boro mostron acto

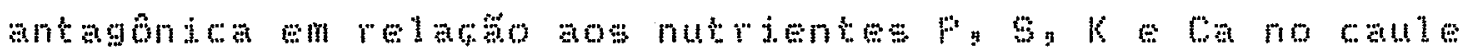

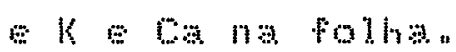

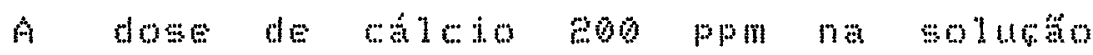

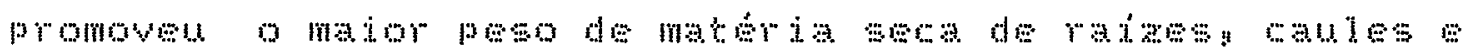

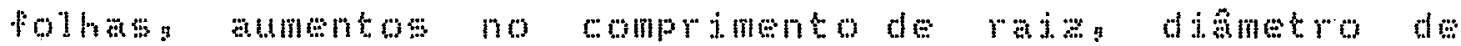

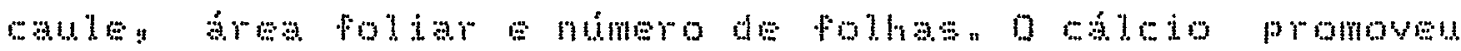
acumulo de todos os nutrientes nas raxzsa caules a follas mostrando anto antagonica com os nutrientes Wa $P$

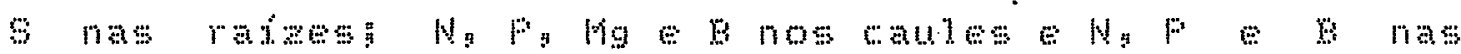
polnas e motrou aro sinerates com os nutrintes o nas 


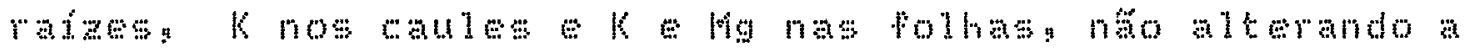

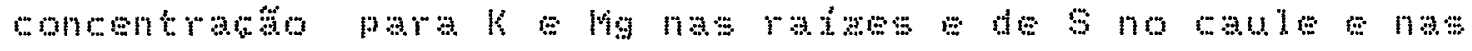
polhas:

Wro houve efejto da interabo ba $x$ a sobre nenhum dos parânetros aval iados.

En orden decresente os resultados medios de concentrato somulo medios de nutritentes foram"

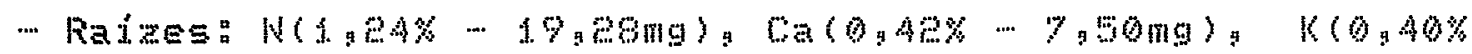

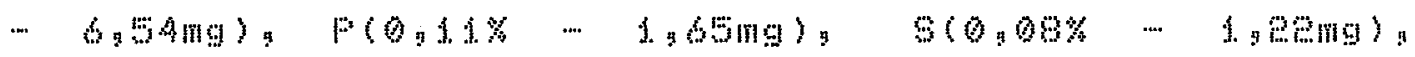

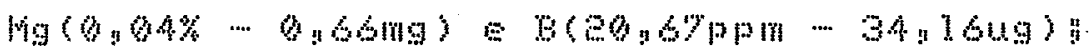

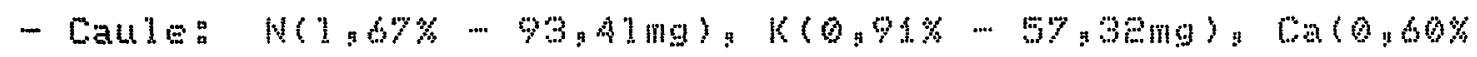

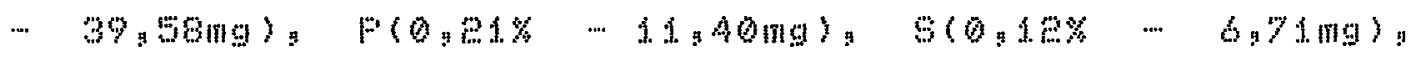

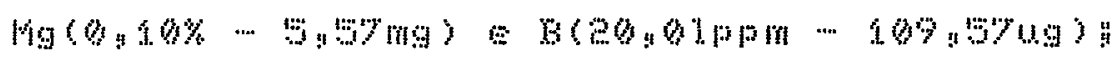

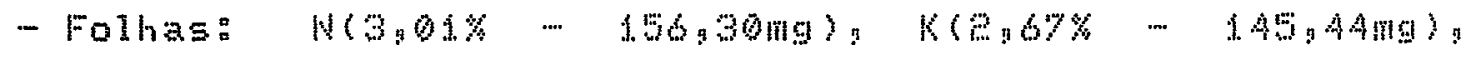

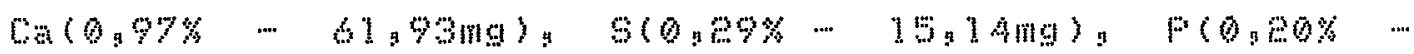

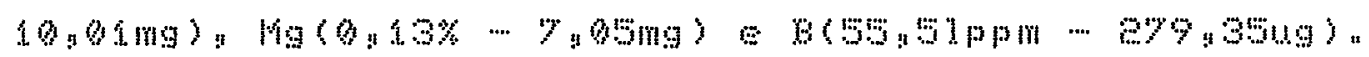




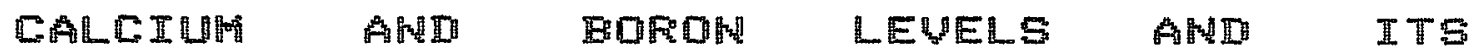

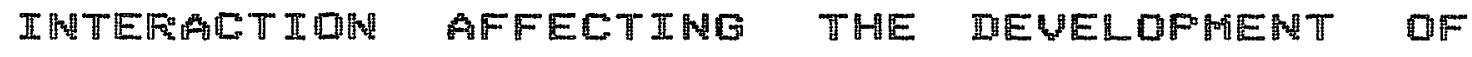

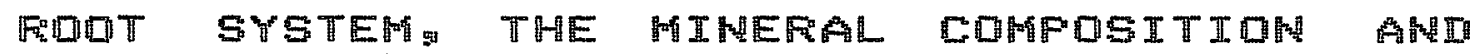
THE UTGQR DF RARGFUR LIHE TREE ELIIZZI IIIMUID

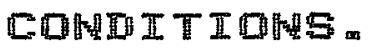

Author: HÉLIO GRASSI FILHO AdYiSET: RR: ARTONIO ROQUE RECHEN

\section{SUIHARE}

The puppose of this work was to study the effect

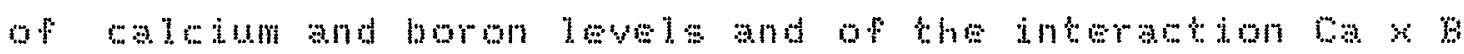
on the development of the root syserin on the inineral compostion and on the vigor of the rangur lime trose

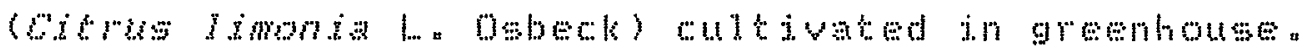

$$
\text { sedings from a nursey of the t.agendo }
$$

Experimental Fang were used. Thes were grown in 31 pots

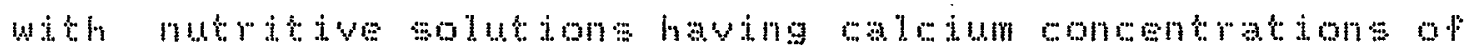

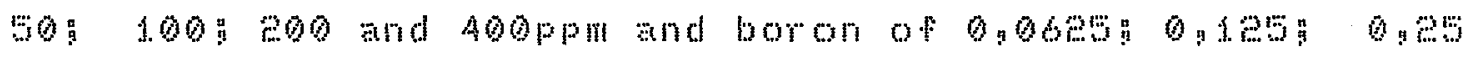




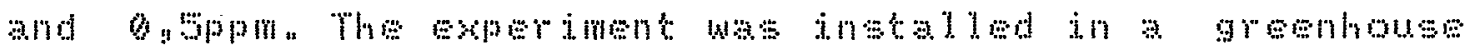
of the boj seiene Department college of Agronoms

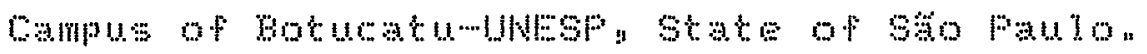

The experimental design was in a randomized blocks with ib treatments assigned in watorial test.

The interpretation of the study was done after Swmonths of the plants development ahen the following parameter: was analjed: drs matter weish of rootsa trunk and lowes rook lengtha trunk diameter foldar arean number of leaves and plant chemical analysis p for the determinetion of the nutrimts nitrogen phosphorus: potassium calejum magnesiumg gulphur and boron.

The a gom boron dose in the solution lod to the Iongow Iength of the roots boron showed antagondsm utith the mutrionts $F$ a $K$ and oa in the trunk and with $K$ and Ca ju the leaves

The eoppan and dom dose in the solut ion promoted the highest increase in dry mateor weight of roots trunks and leaves as well as in root length trunk diametern

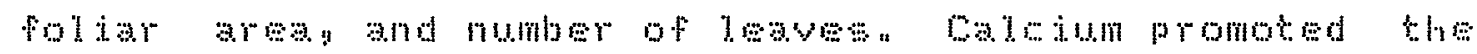
accumulation of all mutrients in the roots trunks and Leaves and expresed antagonism for the nutrients $N$ p and $s$ in the rooks $N_{g} F$ lig and $B$ in the trunks and $N_{a} F$ and $b$ in the losves it a 1 so showed sunorgism for the nutrients $g$ in the roots $k$ in the trunks and $k$ and ing in the leaves it didn't alter the conentration for $k$ and wo in the root: and $s$ in the trunks and leaves. 
There waste ans offect of the interaction cas B for any of those eyaluated parameters.

The average results of concentration and nutricnts acumalation observed are presented in decreasing order as follow:

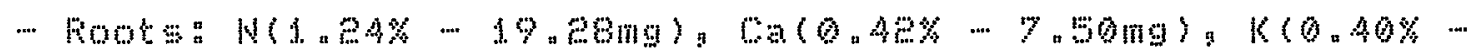

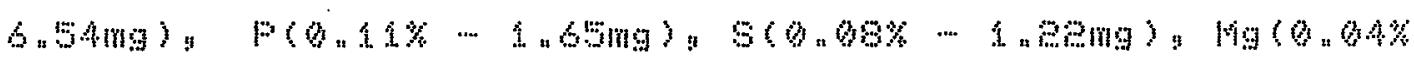

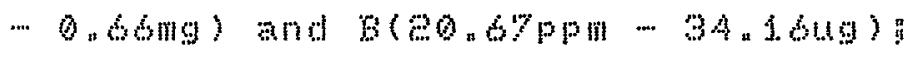

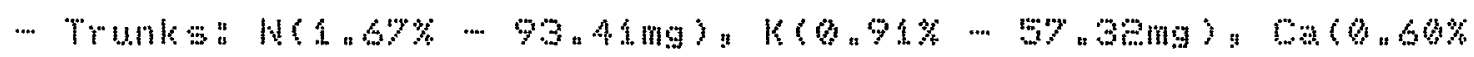

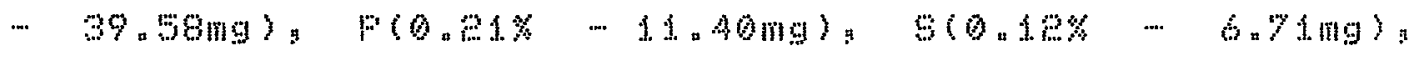

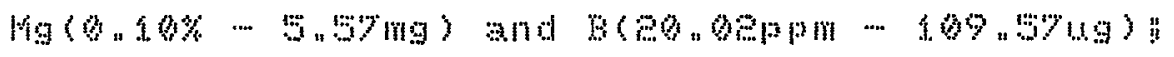

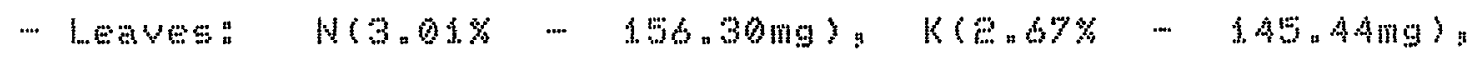

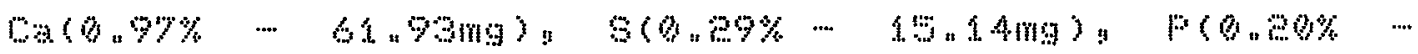

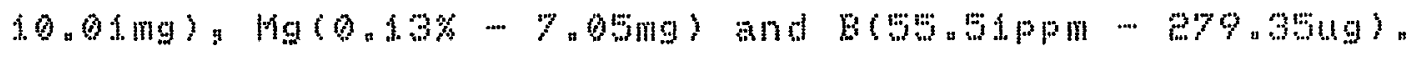




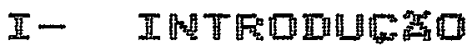

o Brasil \& hoje un dos mares produtor exportador de suco atrieo do mudo. mpho (1987) avaliou

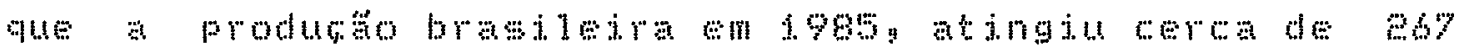

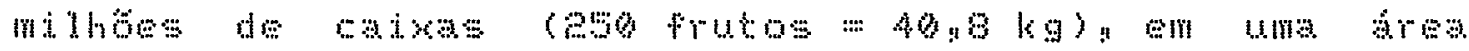

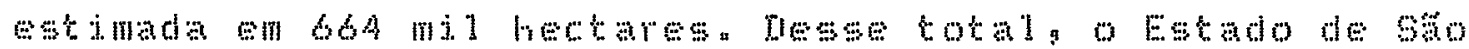

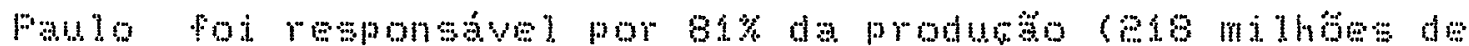

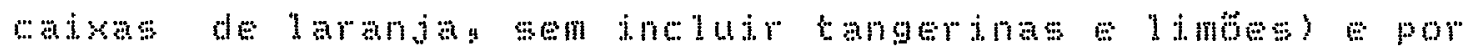

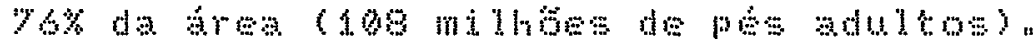

For outro lado a agroindustris de sueo eitrico tamben apresentou notovel. desenvolumento a partir

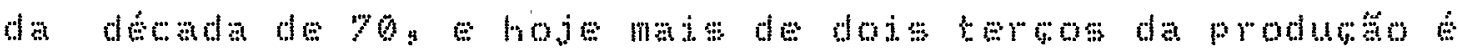
industrialiamo exportada na fom do suo concontrado congelados whando a movimentar divjsas que atjojman a

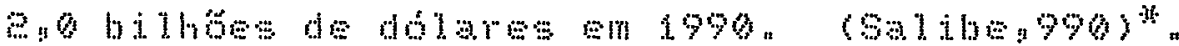

SALIHE: A.A. Informatăo pessoal. 
Tnumbras pesulsas tem demonetrado que:

quando diferentes especies vegetas ou ate mesmo

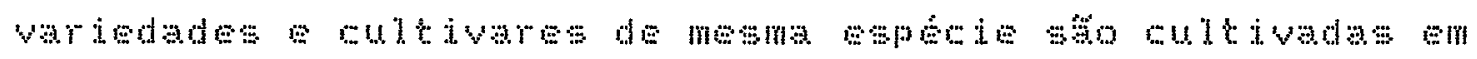
un mesmo substrato e sob condicosede cultivo identioas

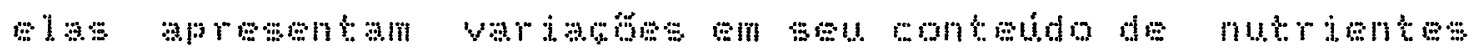
minerais. No caso de plantas citricas é necesario conhecerose as exigneias minerais ou comportamento nutricional das varjedades utilizadas como enserto e portacenxerto sue forman a arvore compostan

mesta pomas varias caratoristoms apresentadas pela copan podem ser moditicadas pelo portan encerto tads como vigor precosdade de produgton

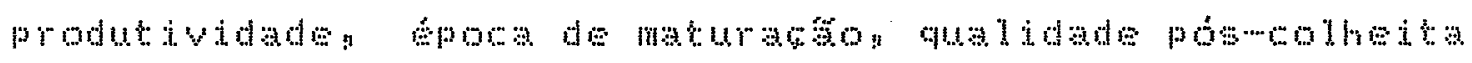
dos prutos fertilidade do poleng composicto orotinica inorgandes das folhas frutos capacidade de absorato mintese de composos organdos a utilizato de nutrientes

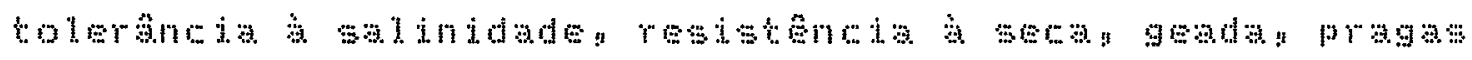

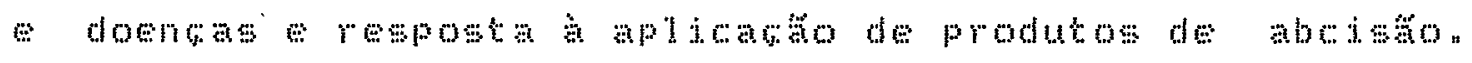
Dentre as influmedas que a cops overes sobre o portacenceron as mase evidentes sán quanto desenvolvimento do sistema radicular resisteneta ao prion

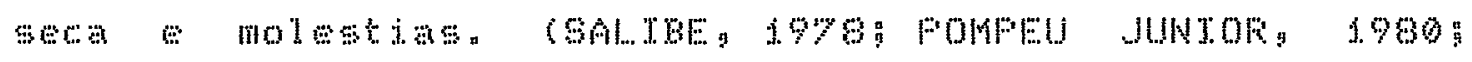

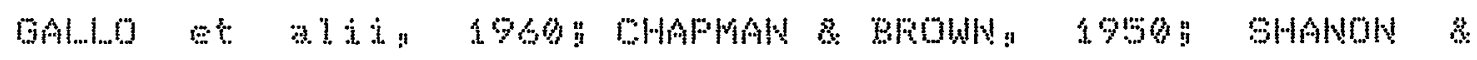

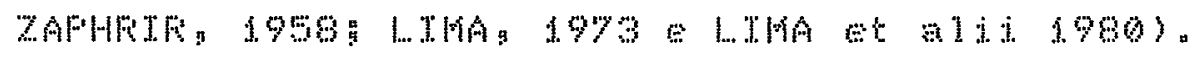

Esse variacos podem wim parte $=$ explicadasy pelas diferengas apresentadas pelo sistem radicular das variedades utilizadas como portackmerow ou 
sejan variodades que aptesentan melhor desenvolvinento no s.stema radicular aue outras a podem absorver maiore: quantidades de nutrientes o também apresentar exiancias diferentes de un ou majs nutrientes. outra explicacto para o fato.

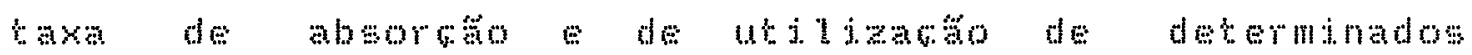
nutrientes.

Foums gaso as pesulane sobre o

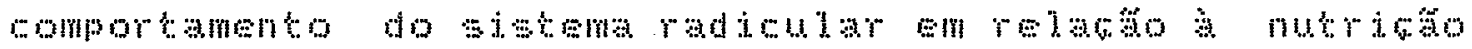
mineral das plantass e com relacto ass plantas citricas

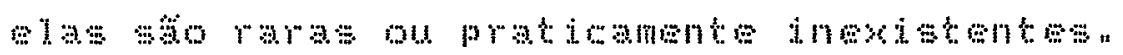

Atualmente os resultados das pesquisas tên mostrado cendsucias que indiom que o problema do dewlinio das plantas citricas están estreitamente ligado a desordons

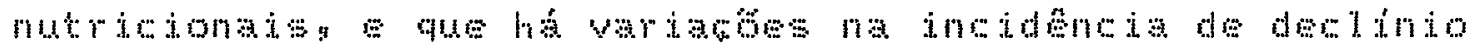

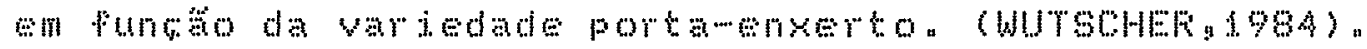

Essos informabes vem reforar a necosidade de estudos bistcos (em condiobs controladas) sobre a influsncia da nutrito mineral de diberones variedades portankerto; ou seja sobre o desenvolvimento do sistome radicular absomo de nutrientes relaso mutricional trans losacto evigor da planta "

absose que para obter uma planta vigorosa é Hundamental a soolha de un porawenxerto adequado a que um dos aspectos importants: 8 o conhecimento do desenvolvimento (ou comportmento) da variedade porta mperton em relasto a suprimento de nutriontes. 


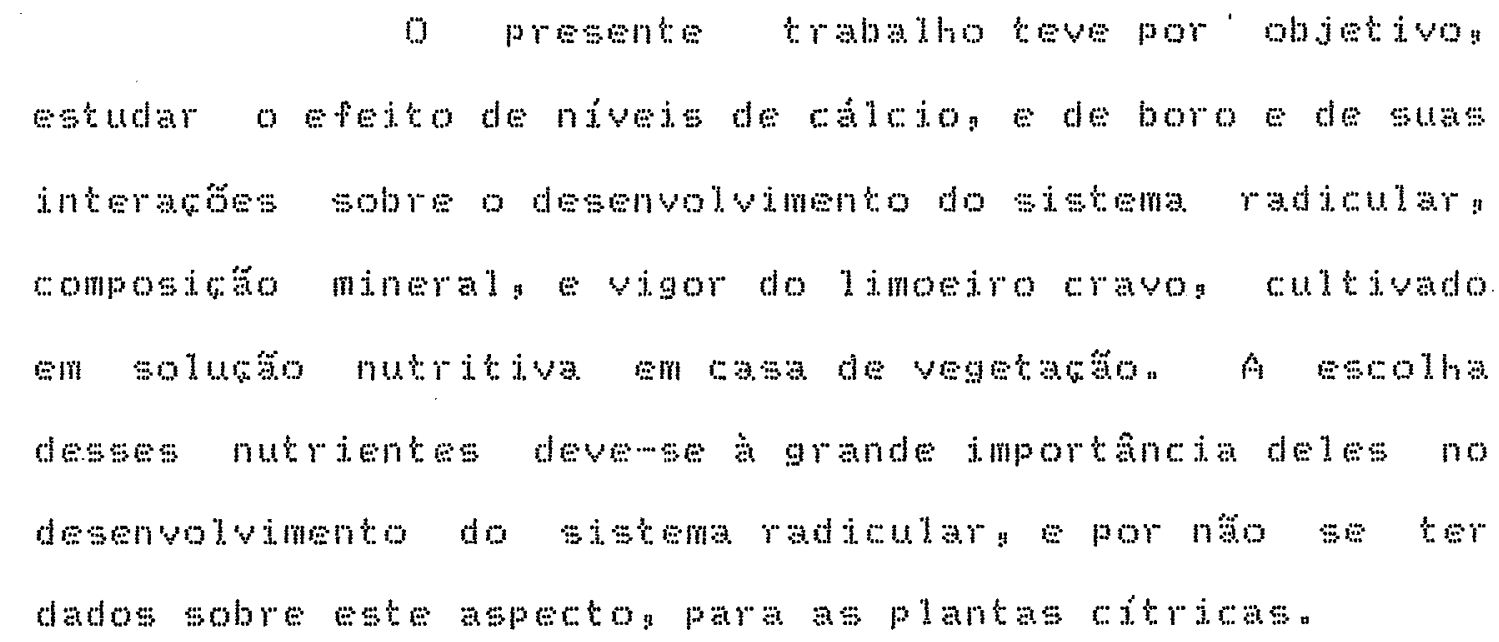




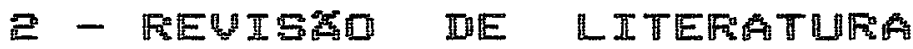

\subsection{Estudo de Porta-Enxerto}

Wá ons comprovada a inflüncia da

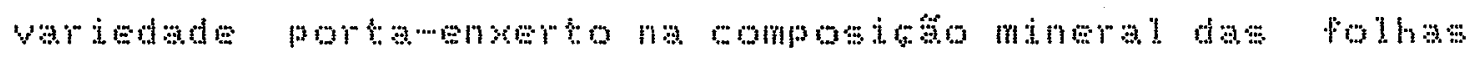
da vardedede copa em citros. pos diverose experinentos jâ rendados varias teorias suratram para explinar o

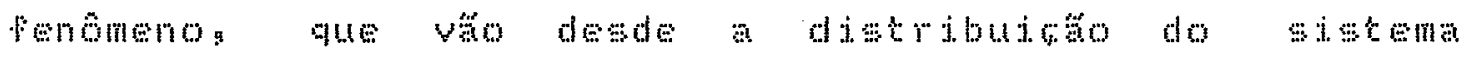
radicular da planta portamenter ate a seletividade e a capacidade de troma de cátons das rades do mesmon influeneiando assima na mat ou menor absorato de nutrientes a (mouneo rs: 1989)"

MOFi: IfA, (194) destacia amportancia do desonvivimento no estudo de portameneros a relata a instalabo de un ensaion com doze diferentes pontan'

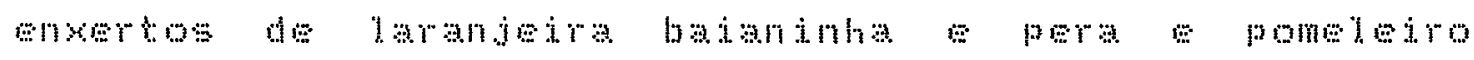

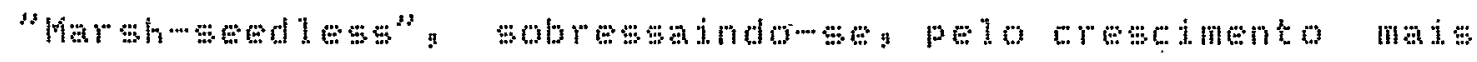
rapdo a limedre da persian limosiro rugoso e limosiro cravo 


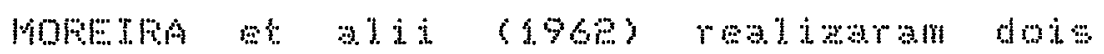
ensaios rom portamenxertos para limosiro eurekan em

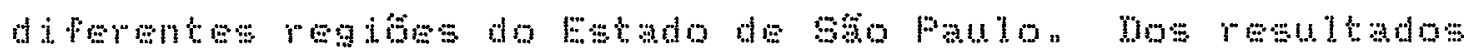
de una strie de nedidas bionotricas os autores

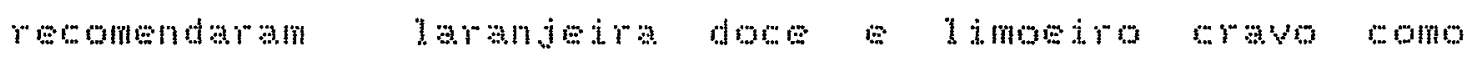
portamentero para essa variedade de limosiron

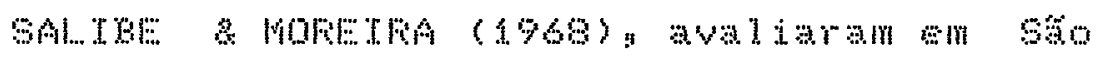
malo o comporamento da laranejo barkon sobre 7 diforentes portanenertos w presenos do virus da tristeran os resultados mostraram que dimoedro cravos

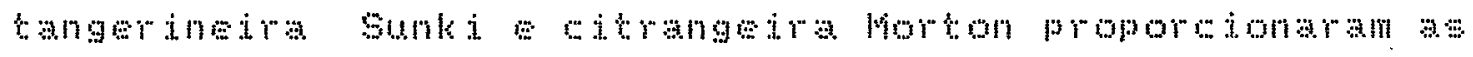
maiores produces de laranjeja barón os autores monstaram anda que estestres portawaresos mais produtivos pomam exatamente aqueles que proporoionaram

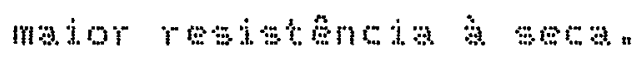

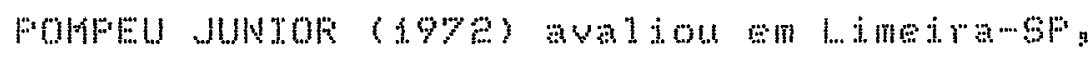

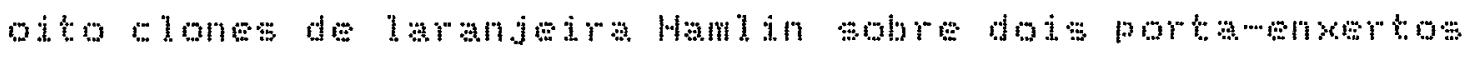
(limosiro cravo e laranjera capira) o autor constatou que as plantas de laranjeira Hanlin apresentaram en median major producto menor volune de copa e mator productio por metro cubico do copa quando moneradas sobre limotro cravo. Fesultados semethantes foram obtidos por Rek

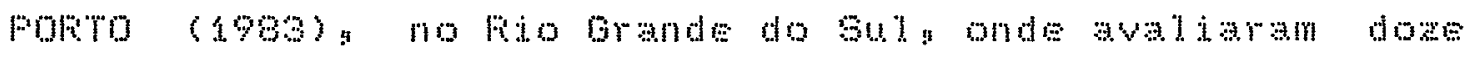
portankertos de laranja Handin. os resultados revelaram que as matores produbes foram old das nas

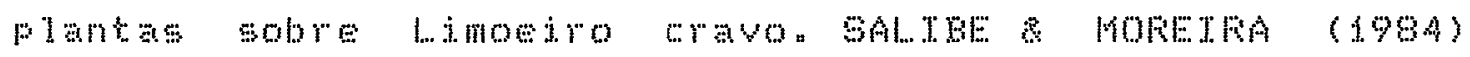
relataram os resultados do diversos experimentos de portan 


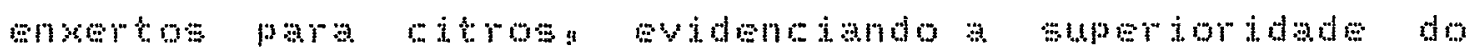
I inoeiro cravo.

Me acordo com TEOFTLO SOBNHWH (1979), a planta de limotimo cravo é de pote medio e bastante Froduciva Apresenta oito a dez ramos prineipais

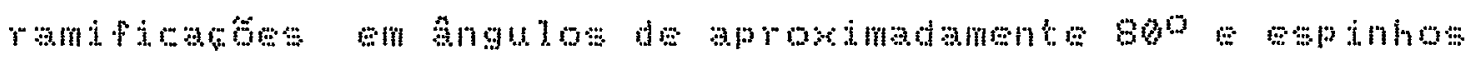

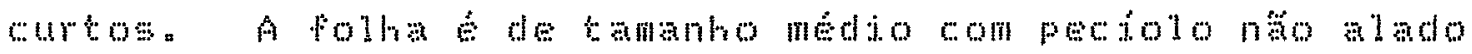

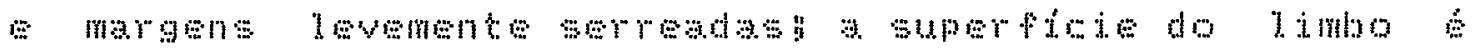
molulada de cor verde claro a a cor do broto éboneadan

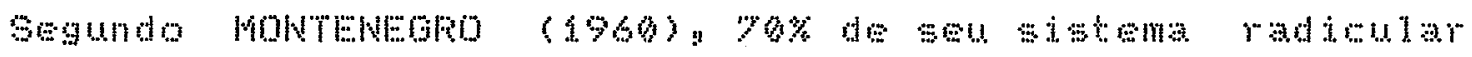

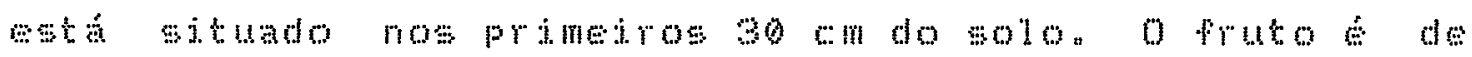
tomanh medion com peso a rodor do 80 a 90 aramas a

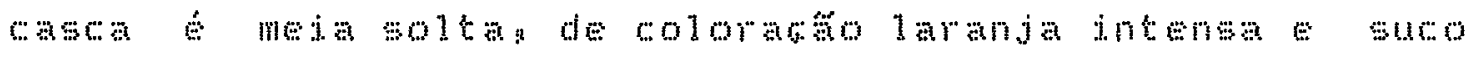
Acido A maturato ocore de abril a mat As semants: sto pequenas an humero de dez a qunze por pruto e doze a quinae mil por quiby poljembronicas com cotiludones esverdeados e que conservan relativanente bem seu poder grminativon

Como já se afimoun esta variedade porta enxerto é hojen a majs utilizada na citricultura paulista estrodo presente em mas de $90 \%$ dos pomares comerojais.

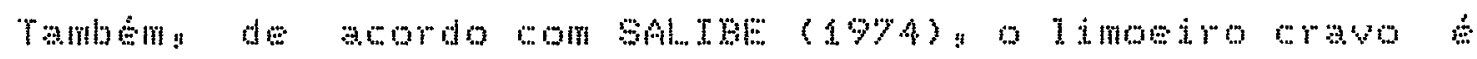
wetiarado como portacenxerto na Tndiag Argentina Fetados

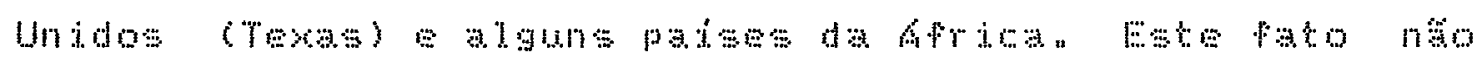
se deu por acasos mas sim devido a uma serie de parores que lovaram os wivetristas e chroultores a oparem pelo Iimosiro cravo. A plantala apresenta grande vigor e 


\begin{abstract}
wedente desenvolvimento no viveiro. po plantas

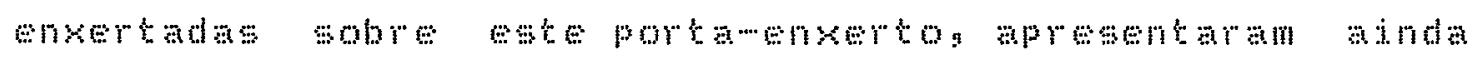
precosidade de produsăon produsto de frutos com boa qualdade eso mulo resistentes a seca
\end{abstract}

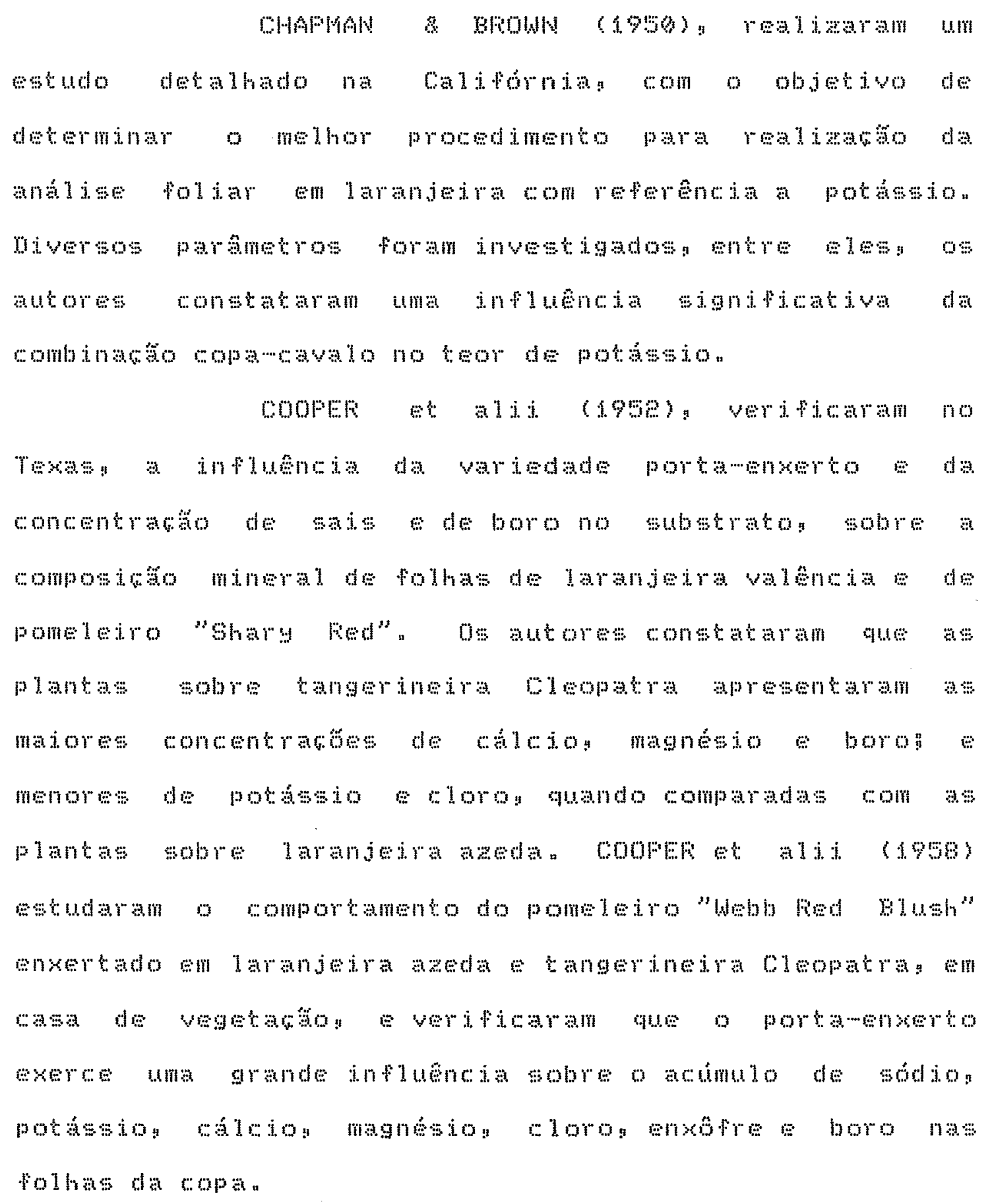


Atrave da malise poliar balno et alit

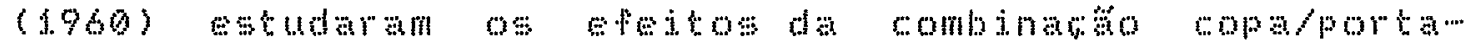

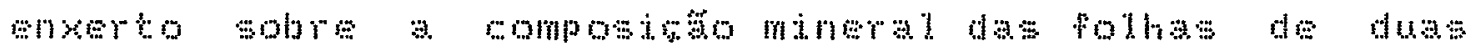
variedades copas enxertadas em winos diferentes porta

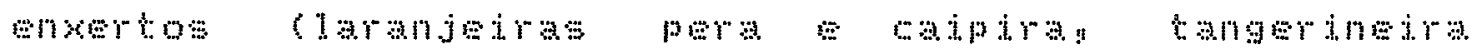
oleopatra limosiros eravo o rugoso nacional) as autores mostraram follos do eielo da primaveray do ramos Putheros: as auds foram analisadas para os teorse de

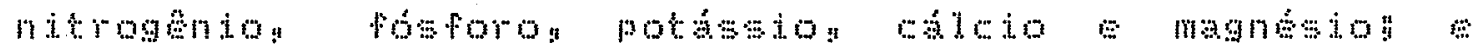
observaram que o portanenerto exereu intluencia na

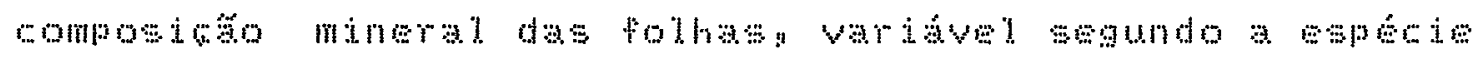
e obtueram diferencas significaras nos teores de

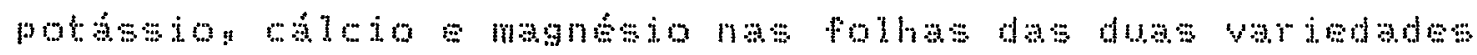
cop: $:$

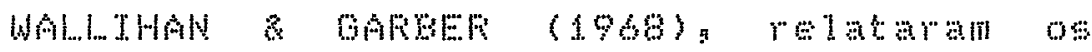
resultados de seprimentos dosenvolvidos na califoria envolvendo plantas jovers de laranjeja doe e laranjejra azedan de pe franco o enxertados entre sig sobre abstratos contendo diferentes conentracos do carbonato

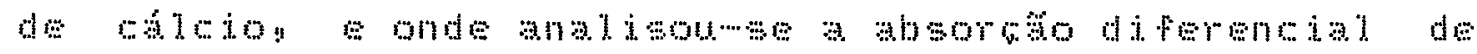
ferro pelas plantas Fela andise "ular das plantas os autores constaram que o teor de ferro nas plantas dependem pelo menos de tres fatores. 1) la quantidade de ferro mbsovidan me varta de acordo com o tamano do

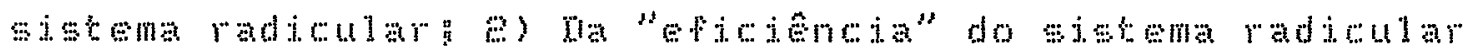

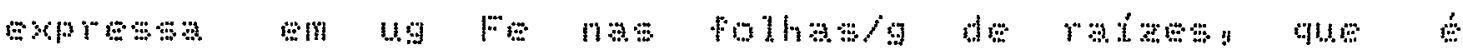
sonsivelmente diminuda pela presenaa de cacosa 


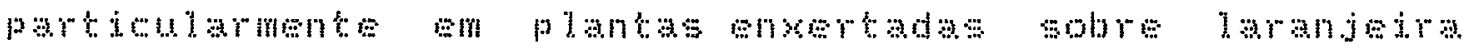

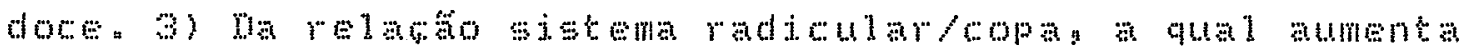

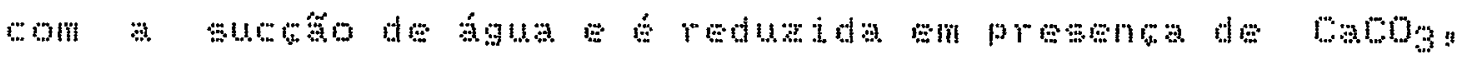
Es:a ralaso mostrouse mator nas plantas sobre larandetra doce. do que nas sobre laranjejra azeda.

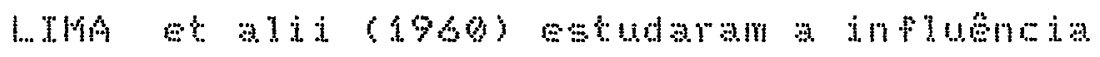
da combinatio copaporta enxerto sobre os teores de enxofre e boro sm follos de ramos prutiforos do eino variedades copas enxertadas ew cinos portankentos

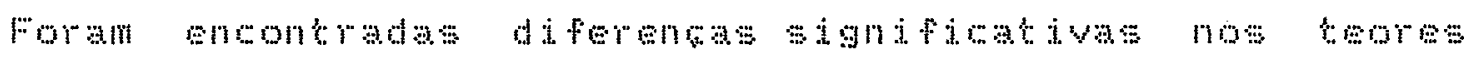

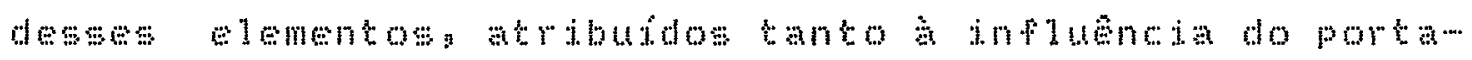
enxerto momo da varedade copa salientando an o

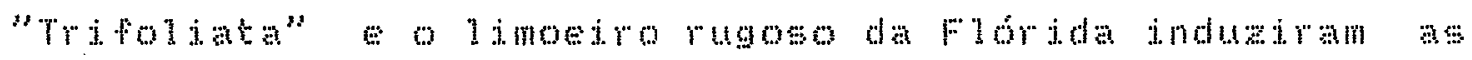
matores concentrabes de bono o o limostro wrave as monom. A laranjeira capira induziu as maiores conentrocose do enxofro.

Wursenin (290e) estudou a influeneda de

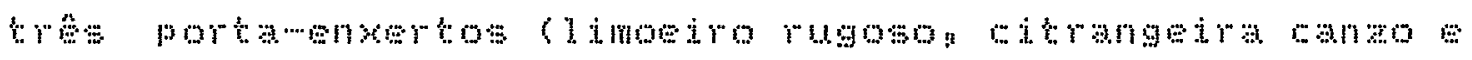
laranjeira azeda) sobre os niveje de nutrientes em folbas

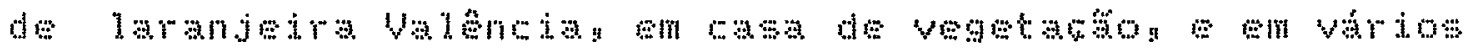
substratos. 0 autor verilicou aue tanto o substrato como portacenxerbo fotaram os teones de nutrientes nas follas da copa Houve diferenos significatiuns nos toores de

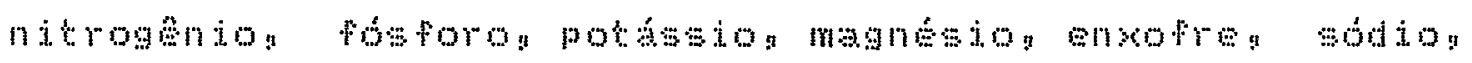

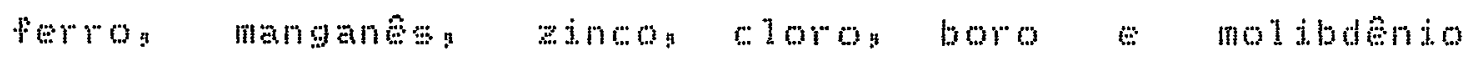
atribudas acs diferentes portankentos. 
MoUngo fo (1989) sstudando a difluencia de ojo diferentes portankerros no vigorg producto productudade das plantas nas concentrabes do maro o micronutrientes nas folhas de laranjejia perag tondo

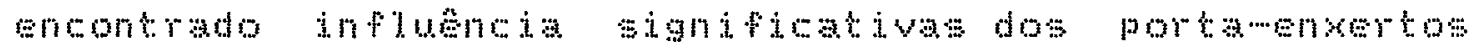
nos teores de potássog caloion manesiog forros manganes

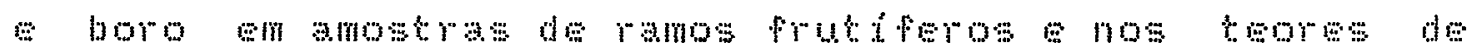

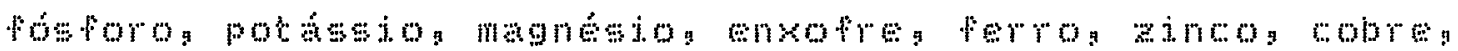
mangans \& boro nos ramos nä prutiferos os nutribntes que melhom explicaram a variago na productio foram

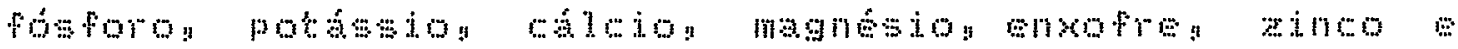
cobres sendo que os portamenertos tangerineiras Sunki o

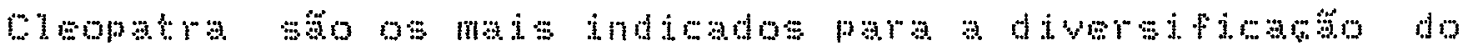
limoedro eravo cono cavalo para laranjeira peran

Enbora mujtos trabalhos correlacionem portawnerto com conoptratio de nutrientes na copa

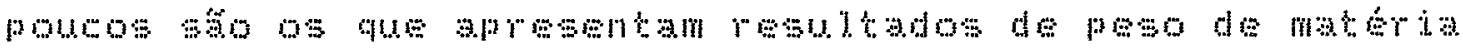
secag area poliar e mamero de folbas om citros e doses SMTH (1969) relata que un pe de laranja pode produzir wm nedia 50.000 follas por ano corespondendo a $10 \mathrm{~kg}$ de

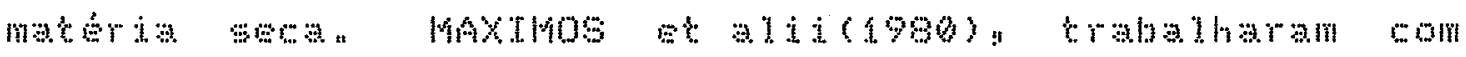
mudas de limoejro rugoso g laranjejra azeda en soluço

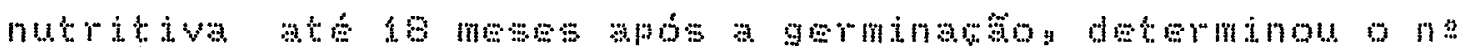
de tolhas por planta o tratamento completo apresentou 137 Polnasplantag as doficiontes wim Na bolnasplanta e as

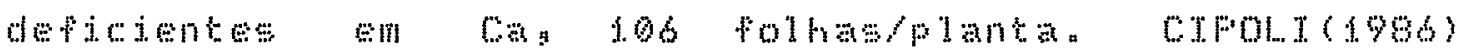

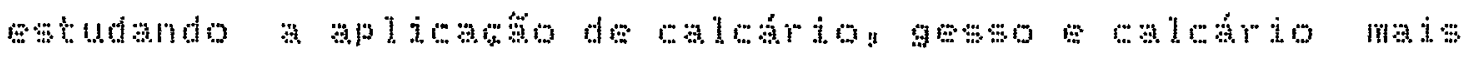




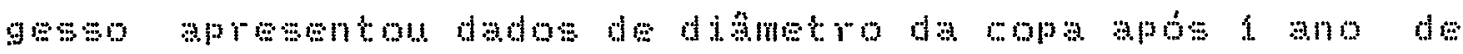
plantion nato havendo diferengas entre os tratamentos.

\subsection{Estudo do sistema radicular}

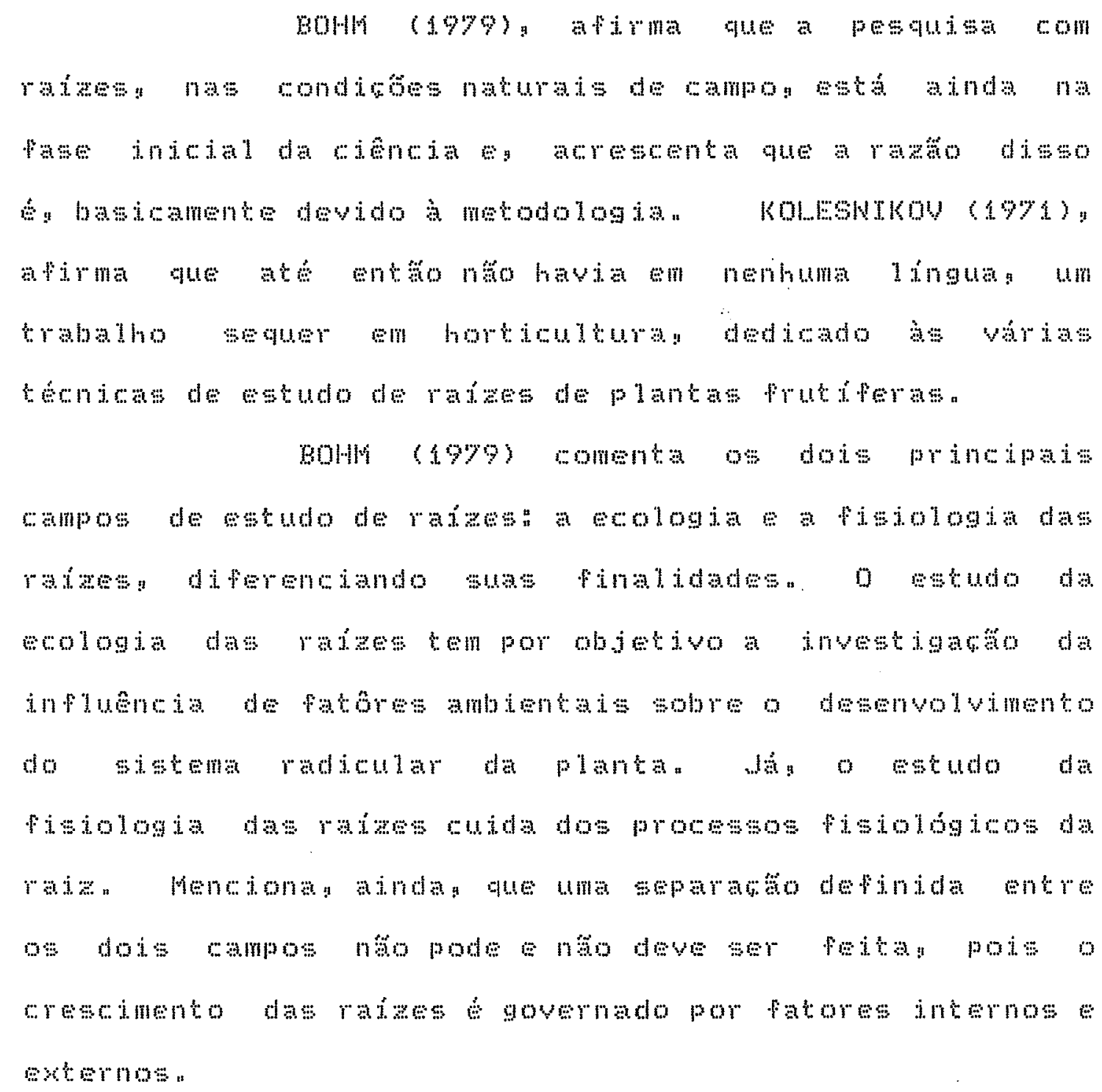




\subsubsection{Métodos de estudo do sistema radicular.}

Os diversos metodos para o estudo de radzes

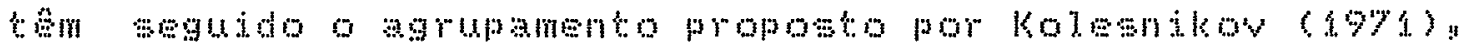

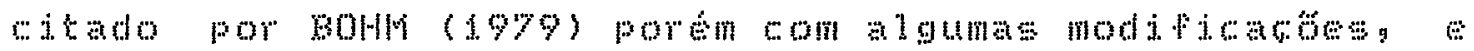

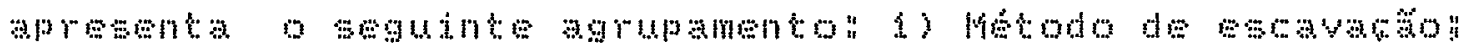
e) Metodo monol itcon 3 Metodo de perfunato com tradon

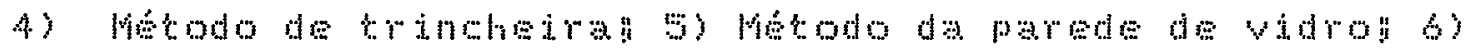

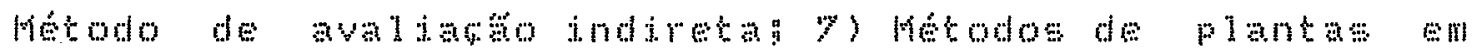

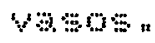

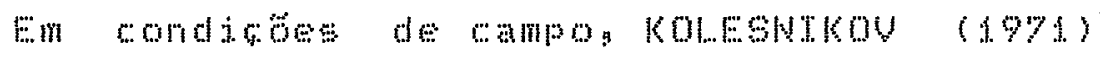

afirm que cada metodo presenta mato ou monor facilidade de mprego e aue o motodo do trado foj utiligado pela

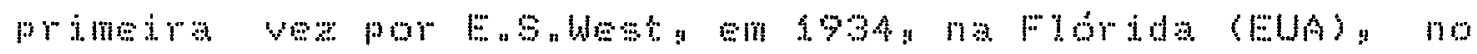
estudo do sistema radicular de plantas citricas razendo sua apreatara sobre o metodo ele afirma que ómodo do trado \& simples e barabo podendo ser utilizado para

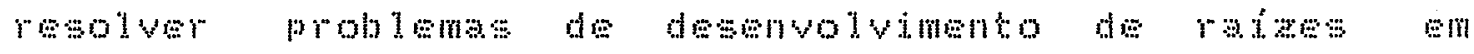
Prutifera.

ATKTNON (j980) menra que o metodo de amostragm com tradon tem sido mprogado promentemente w estudos de rades de arvores frutaferas e outras arvores

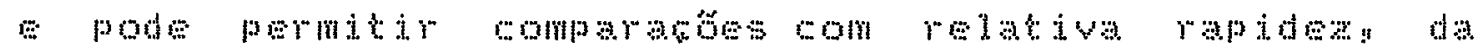

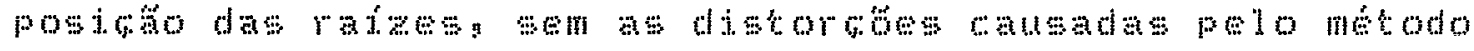
da escangato totaln 


\title{
2.2.2. Peso das raizes
}

\begin{abstract}
BOHl (1979) diz que opeso das ratzes é parknetro mats comum para o estudo do croseimento das

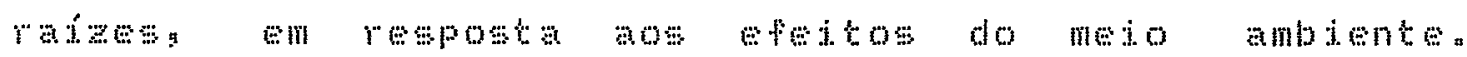

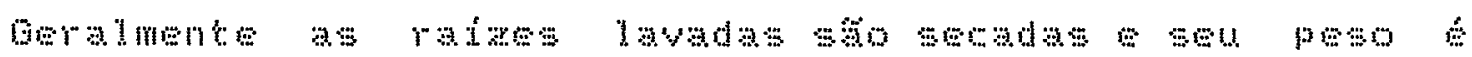
determindo. Informa o atom: que o peso do material seco deve ser proferidon pois muitas informabs conernentes ao crescimento e functo de ratases baseado nelen por ser o mats prociso mplaments aceiton por isso, pode ser validamente comparado.
\end{abstract}

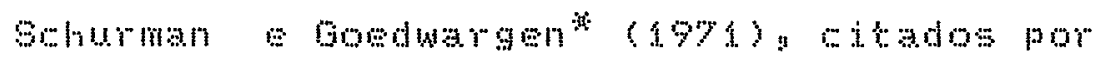
BoH (1979), afirmam que para a determinacóno do peso do

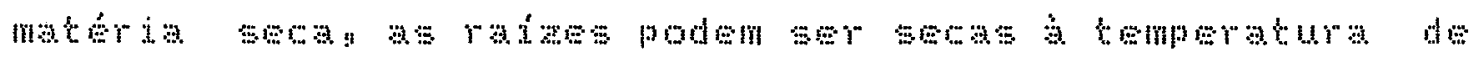
60 a 7500 . Trabalhando nessa paxa de comperatura hat nemesidade de tempo mats longo do que quando so utiliza

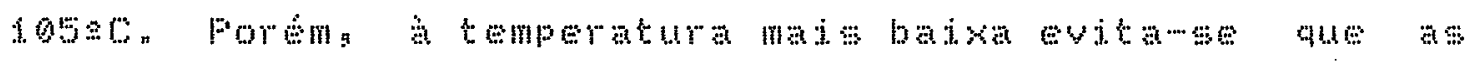
ratass sejam muto fragmontomsan

2.3. Estudo do sistema radicular de plantas citricas

WONEs et aid (19\%7) realizaram um

\footnotetext{
* SCHURMANg J. GOENARGEN, M.A.J Methods for the examination of roots systens and roots. Wageningen. Pudoc Zend 1971. . 66 .
} 


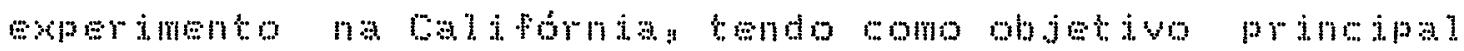
verificar a influencia de substratos con diferentes

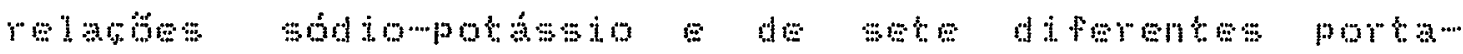
enxertos: sobre o desenvolvinento e o teor de nutrientes nas follos ramos ratase de plantas jovens de limosiro "Allen Eurek" No aue diz respejto no lithimo aspecton os autores constataram aue as variedades portamento exererom grande influencia nos teores de calobon manesion potastio esodio em todas as partas das plantasn MONTENEOKO (1960), utilizando o método do tradou estudou a distribuito do sistema radicular dis laranjeiras Hamling badaninha e peran enxertadas sobre diversos porta enxertos a tambem o watema radioular do péprano de diversos porta enxertos nas condisos do

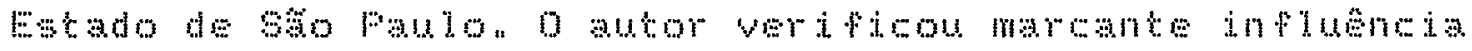
da variedade copa sobre o sistema radicular anando 3 plantas nofo eram ensertadas o sistima radicuiar

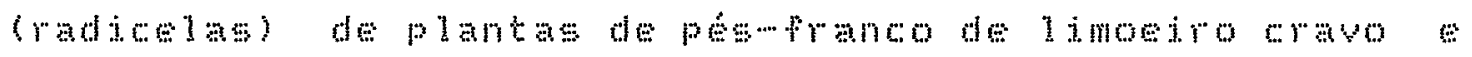
laranjeira aredan mostrouse com mar concentraso junto

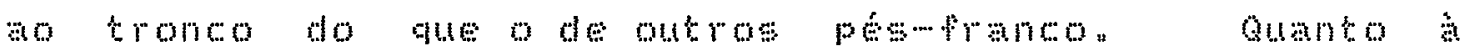

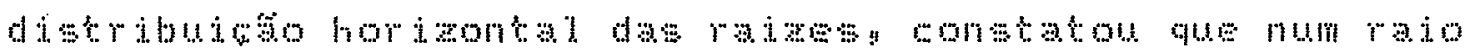
de 2 metros a partir do troneon să whontrados de 75 a $94 \%$ das radicelas " cumbo profundidade constatou que en Arvores com 10 anos $90 \%$ das rases ostä na camada do 5olo que wi ds superficie a profundidade de bocm que para arvores de 23 mos $90 \%$ das ratos esto na camada

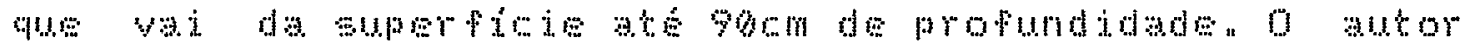




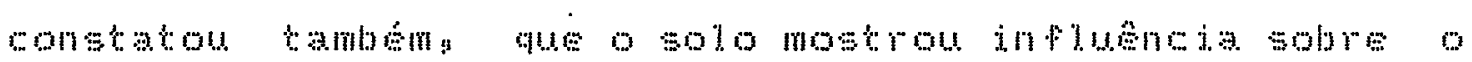
peso das radicelas a distribuiso do sistema radicular.

As0 (197a) estudou o comportamento de wino portarenxertos en vasos contendo solog com adiobes de

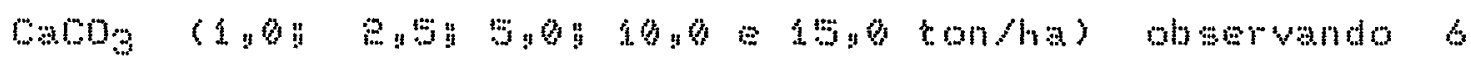
meses mas tarde aue a defindencia de perro apresentonse diferentemente entre os portanenerter de tal modo que a laranja azeda mostrouse a mais tolerante a deficjuncian

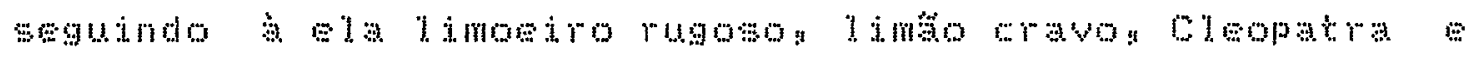
Tripoliatan MELANO \& CABALILEO (1974): estudaram na Espanha a distributaso das ratas absorventes de plantas Eitricas, com uso de radjoisotopos e verificaram a maior absorsto do 30 fr na profundidade de ow 3ocm sendo que para

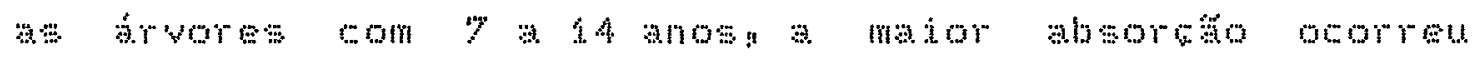

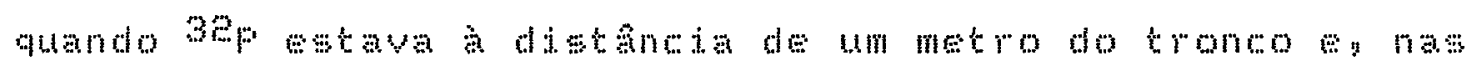
plantas más velhas com trinta anos de ndaden a tress metros do troncon

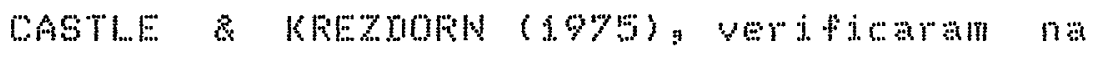

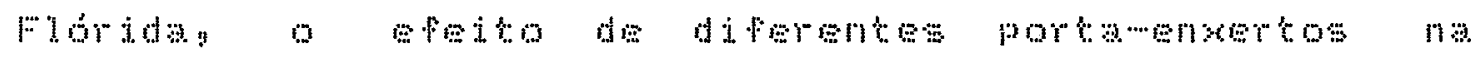
distributato do sistema radcular das plantas e na

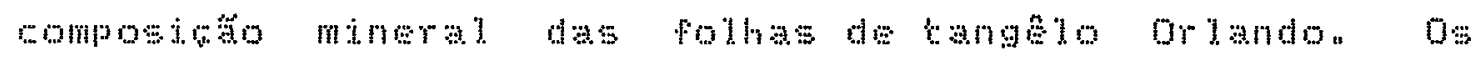
automes conetataram que os teores foldares de nutrientes

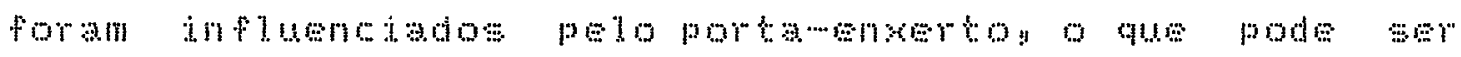

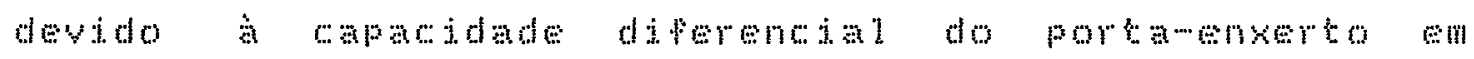

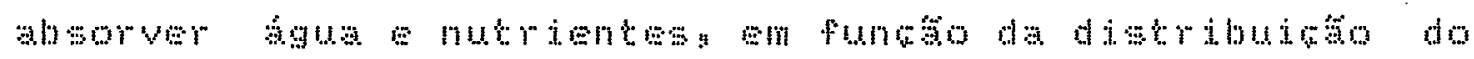

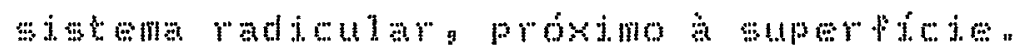




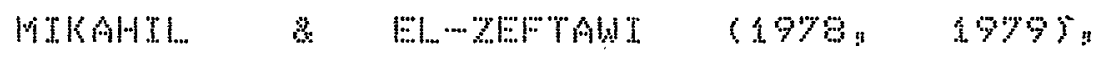
estudaram na mustal ia o efeito de diferentestipos de

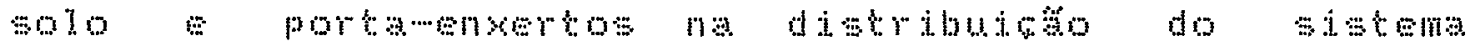

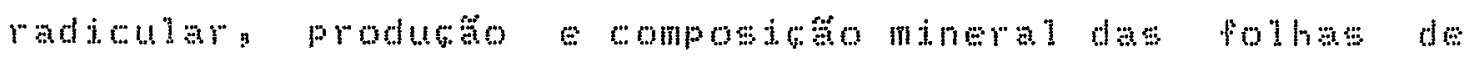

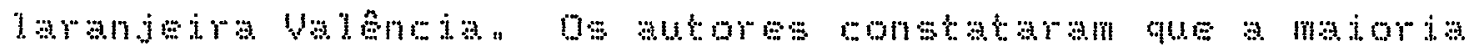
das rataes se encontravam nos primeiros profundidaden independente do portamenkerton

FACE (19\%9) estudou o sistema radicular da laranjeira Watal mom einco anos de idaden sobre trese portamkertos de citros ew solo Fodzolico Vermelho: no Fetado do kio de Jansiron o autor encontrou moror porcentual de radicelag nos primeiros eocm do protundidade do solog na area corresondente ao rato de projerso da copan Constrou acentuda diminubso de radiceias abaixo

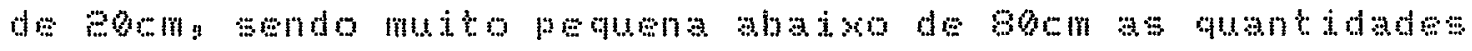
de radicelas. Afirm o mutom que o limosimo volkamerano mostou tendencia a mpresentar mator abundineta de radicelas nas camadas mas superticiads do solo do aue o

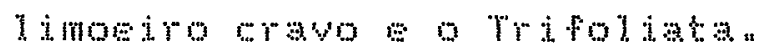

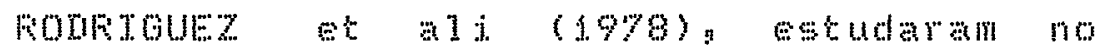

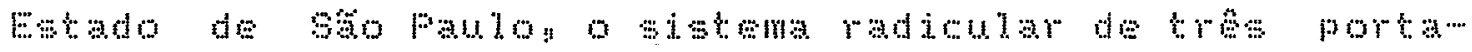
enxertos de citros desde a jad de 3 mesos ate a idade

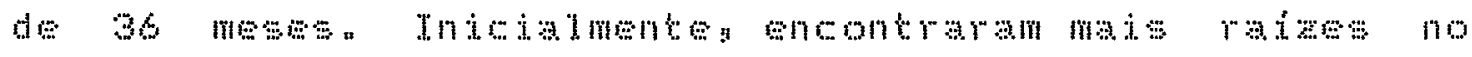

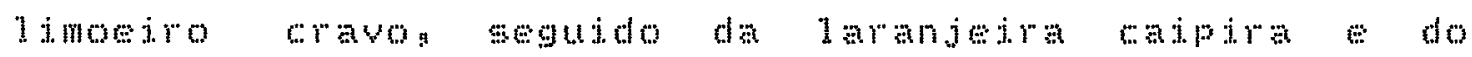
Trifoliatan Houve atrerabes durante operodo estudadou

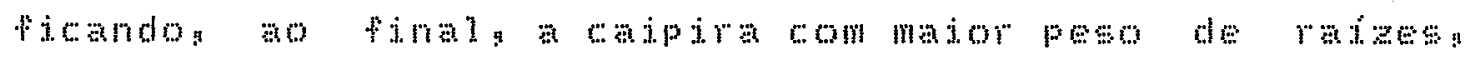

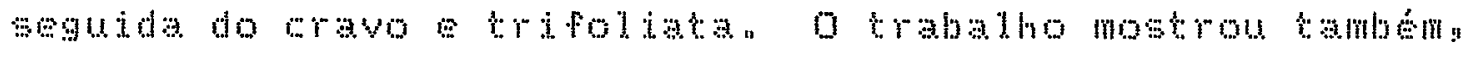


mator quantidade do rafase na camada supertioial do solon existindo entre $0-10 \mathrm{~cm}$ 4f\% $\quad 3 \%$ e $28 \%$ de rafzes

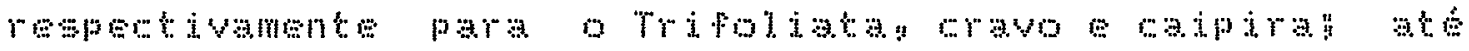
5ocm de profundidadey estavam $91 \%$ das ratzes do Tritoliaton si\% do taipira e $79 \%$ do cravon

DARY 8 WEERT (1980) estudaram o efeito de

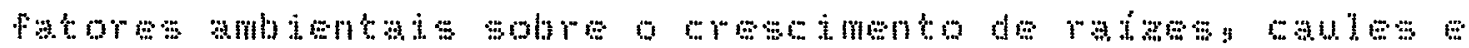

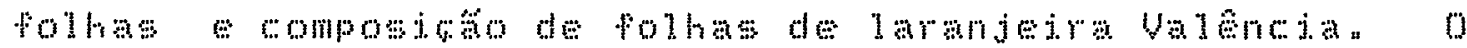
setudo foi realizado em casa de vogetano sondo conduzido

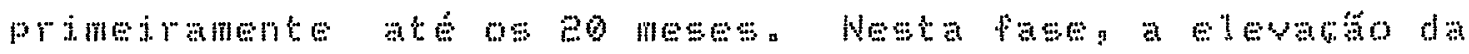

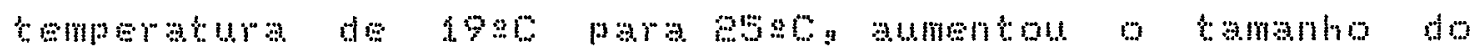
* istema radicular en duas vezes nấo havendo diferengas

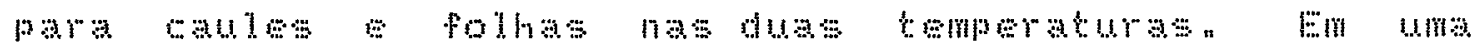

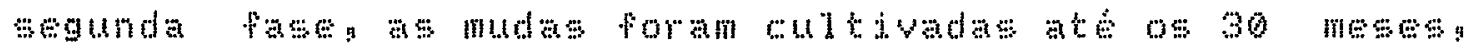

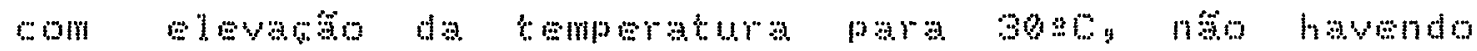
diferengas de cresinento de raiz com a temperatura de $20 \%$

MEDTNA URRATA Et aIj(1980) estudam a

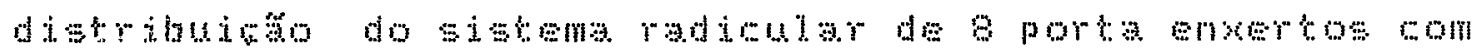
lineira da Fersin com relatio à densidade de ratzos com d.

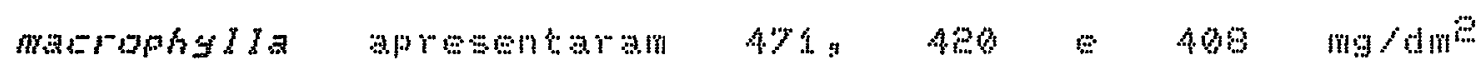

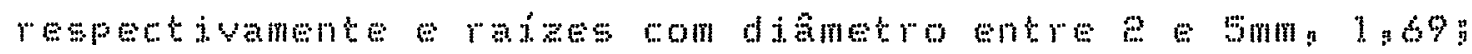

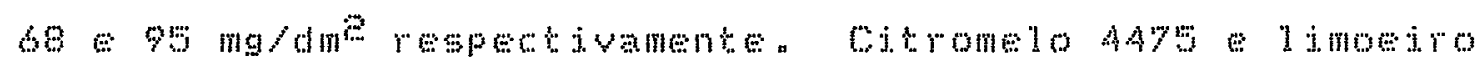
cravo aprosentaram o stetema radicular mas protundon com majs de $39 \%$ das ratzes menores aue emm de diametro abaxo de 50 do 
$p o s$ sem mas de $8 \%$ do suas ratres monores que emm de

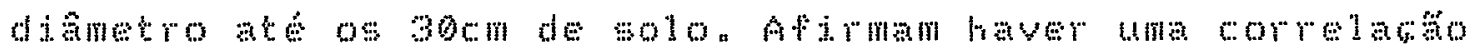
entre o creseimento das raizes o oresejmento da limeira da Fersian

MEITNA UARATA E SEFULTMA TOFEES (1986)

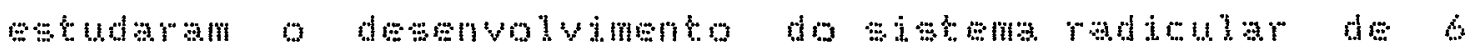

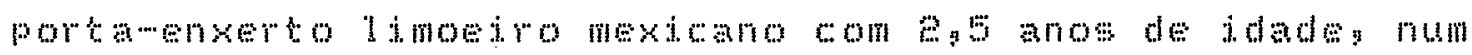

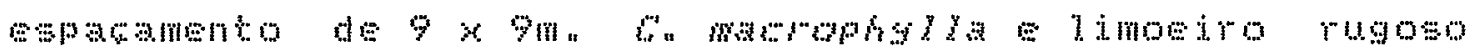
apresentan majot numero de rajase esstema radicular majs

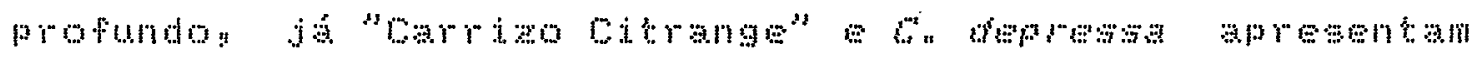
menor munero de raizes o astema radicular mas superficiads " " entre os citados anterionente. A densidade de ratzes en todos os cose foi maior dentro do raio da copan do que Pora dele.

BEHROUMAN et aid $(1986)$ ostudaram o

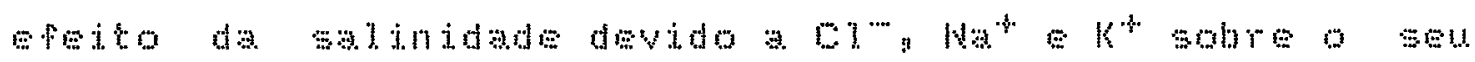
concudo nas folhas wales e raizes de laranjeira vancing hinown Tolor o Tangor Ellendale sobre

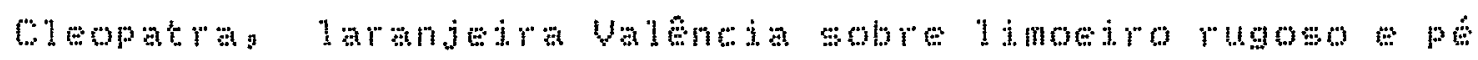
franco de limosiro rusoso Houve diferenga no acumb de Q1 na raz en fungto do portarenxerton sendo nenor para a Cleoparan limoino rugoso limosiro rugoso perpancon sendo estendido para calle folhss Nas condicbes de

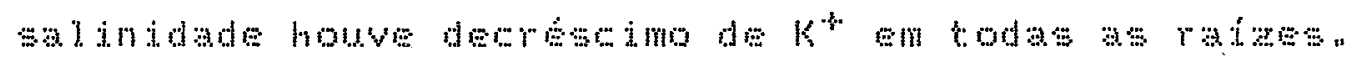




\title{
2.4. Acúmulo de Nutrientes
}

\begin{abstract}
A aboroto de elementos minerais pelo ctros so ds durante ano todon mas borem mator no floresumento a quado se forman follas e ramos novos "

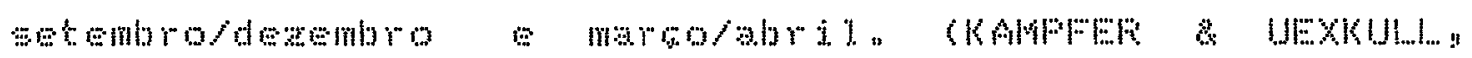
19669
\end{abstract}

As baixas temoratumas atetam negativanente

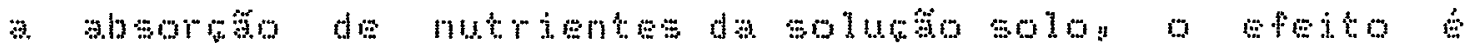
entretanto. menos acontuado no caso do nitrogenio e do majo o mas pronunciado para o potaseio o manesio

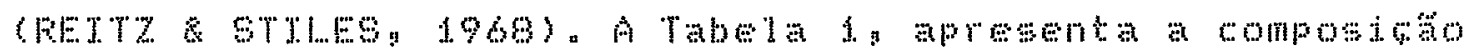
mineral da parte vegetative da laranjoira vense que o wajo \& o elemento dominante seguido pelo nitroganto o potassio e manesion o encopre deve ocuar un lugar intermedirio entre o fosforo o manssion

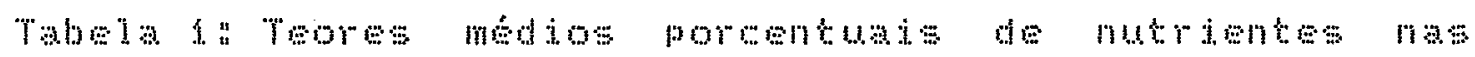

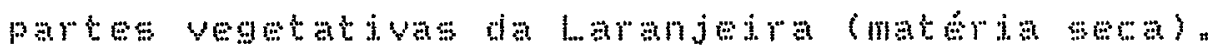

Org ลै०

N

$F \quad k$

ca

$M 9$

5

\begin{tabular}{|c|c|c|c|c|c|c|}
\hline wolhas & e y 2 & 0,18 & $1: 31$ & $4: 20$ & $0,8: 3$ & 6 : 8 \\
\hline Mamos & $1,0 \%$ & 0,17 & $0,1,3$ & $1,8 \mathrm{E}$ & 0,88 & $\cdots$ \\
\hline Tronos & 0.40 & $0,0 \%$ & 6,21 & $6: 51$ & 0.07 & $\cdots \cdot$ \\
\hline 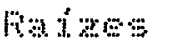 & 0,60 & .... & 0,28 & $0: 10$ & 0,05 & $\cdots$ \\
\hline
\end{tabular}

Fonte: KAIPFEF \& UEXKULL (1966) 


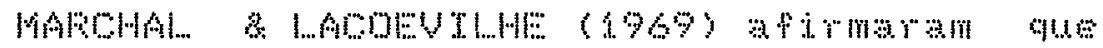

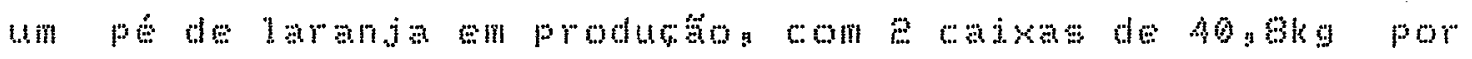

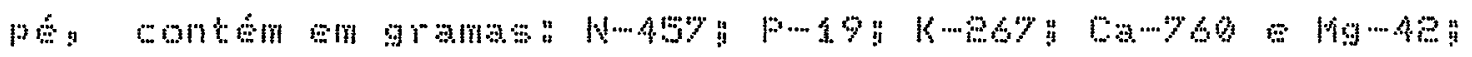

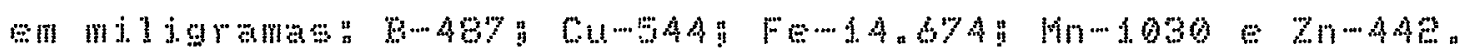

\subsection{Cálcio nas plantas}

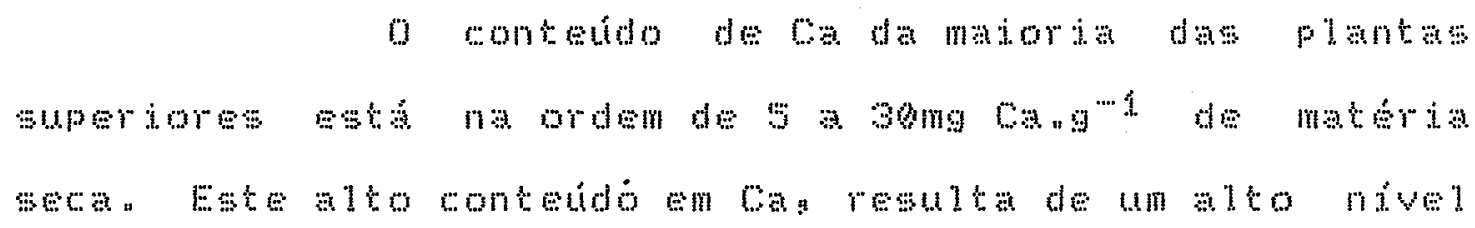

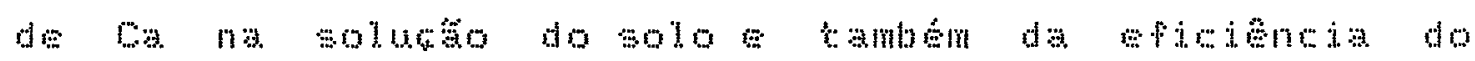

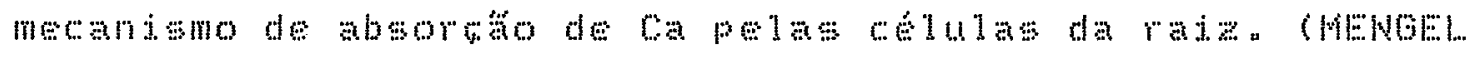

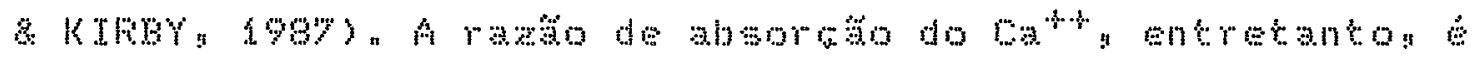

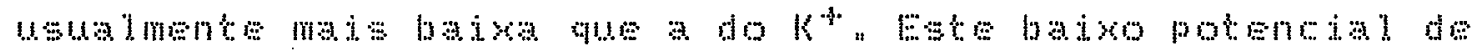
aborato do cat decome do paton aue ase nutriente

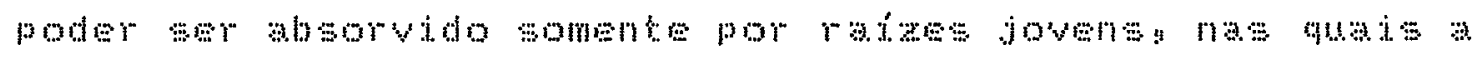
parede celular e a endoderme nô osto suberificadas

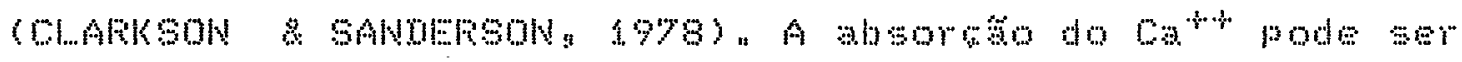
dininuda competitivamemen pela presensa de outros

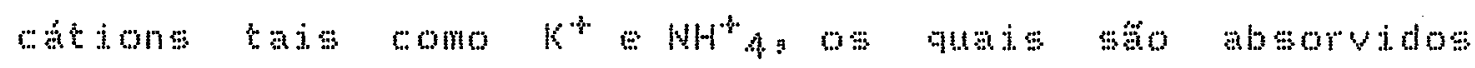

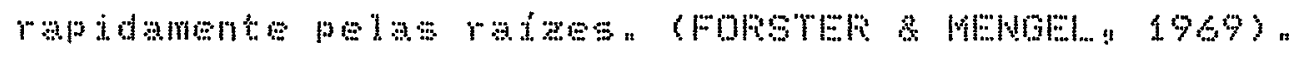

Na plantag o Ca manha pelo simplasto ou

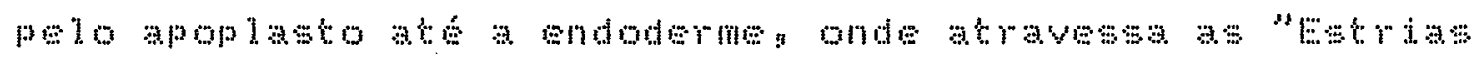
de Caspars" ativanenteg passando pelo saplasto e chegando mos vasos do rilema " No xilema por flurowdemases ou por troca ionica com os radicajs livres da parede do xilema 


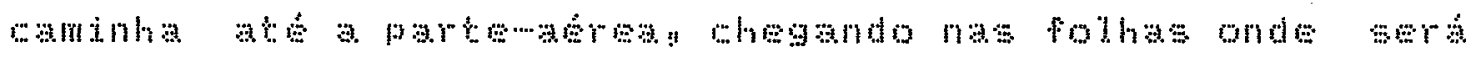
metabolizadon tomandows prationente jovel na plantan

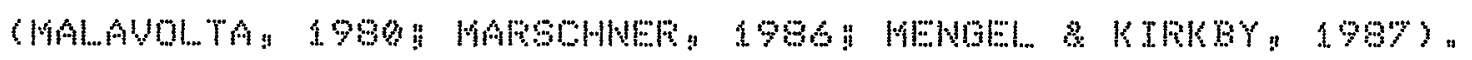

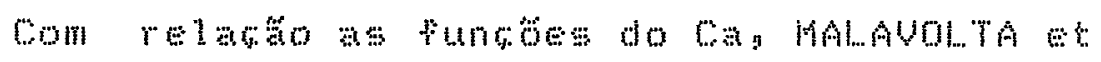

a i i (1989) dividemans em"

1) Estrutural: pectatos de caloio (Lamela modia) carbonato de caleion oxalato de calcion fitato de

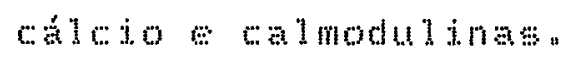

i) Constituinte ou ativador enzimationa ATFuse (aspirase)

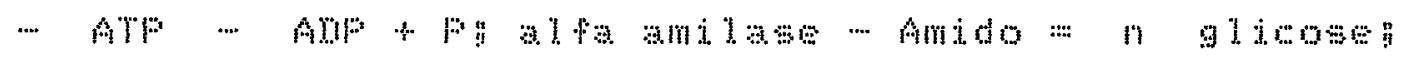

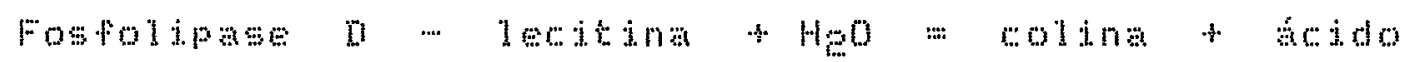

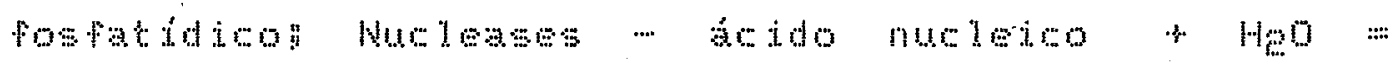
muin leotidios

3) Frocesos " estrutura e puncionamento de membramas:

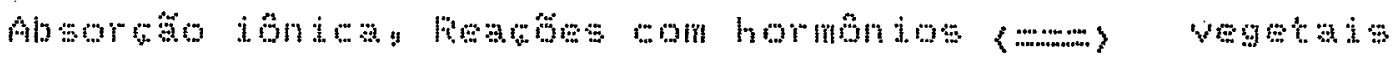

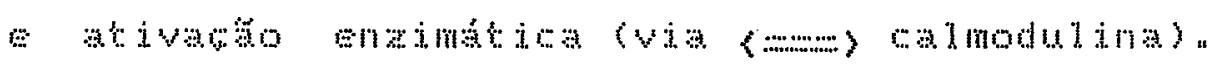

4) Fapeja na formano: fistimula o desenvolumento das

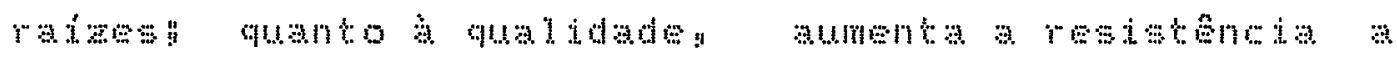

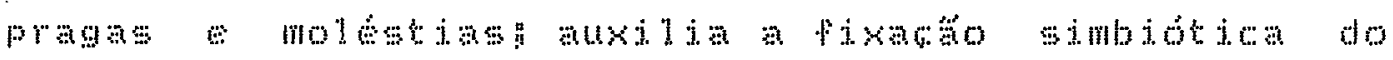
nitrogendo! e amenta o pegamento das flonadas 


\subsection{Boro nas plantas}

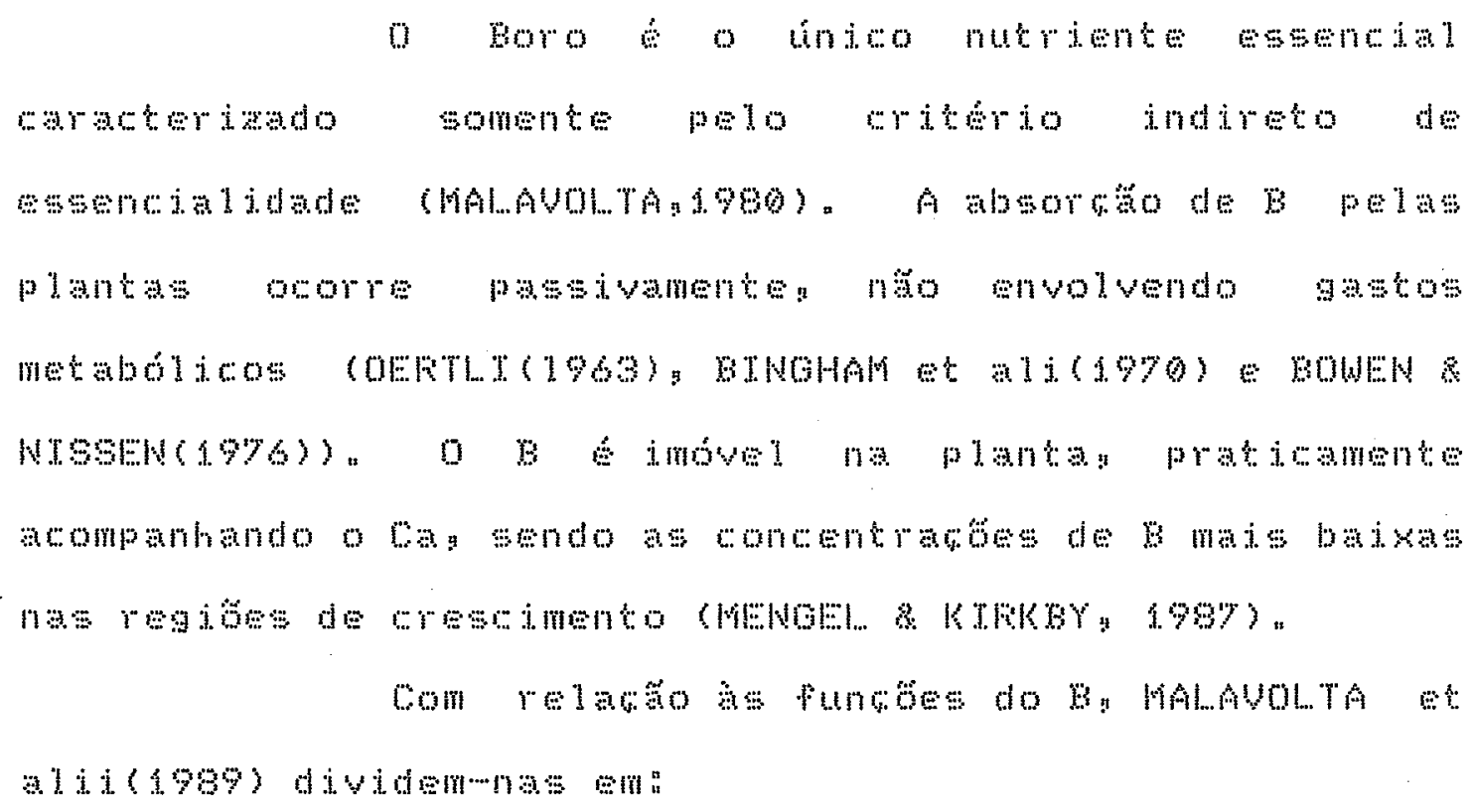

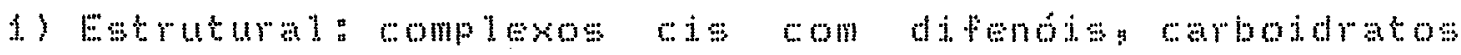
5 a

e) Constituinte ou ativador de ensimas Arpase dis

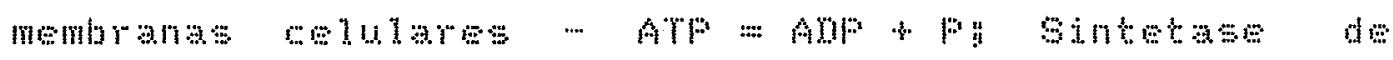

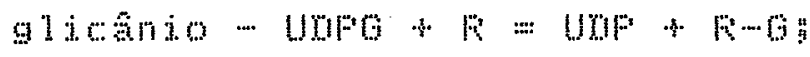

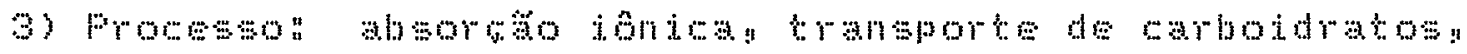
sintese de lignina e celulosen sintese de ádos

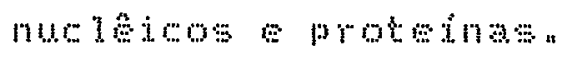

a) Fapol na pomato e na qualdode das colbeitasa germinasio do ando de poben eresemento do tubo polinicog melhor pegamento da ploradag menor 
estrolidade masculina e chomamento do aros.

\subsection{AGão do cálcio na absor̨̧̃o de cátions e ânions}

A concentratoso de Ca na solutäo pode aptar

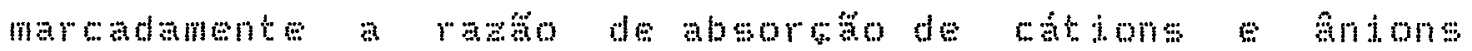

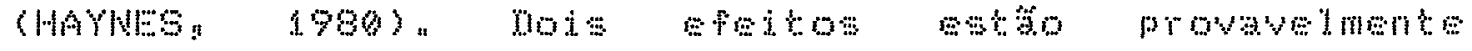
movoldos en primejro lugar baxas conontrabes de ca

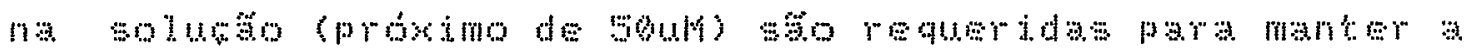
integridade das membranas (EFGTEINa 196e), sendo osto eteito espetico para o ba lem segundo lugara altas conentrases de Ca na solugho podem diminutr a absoróno

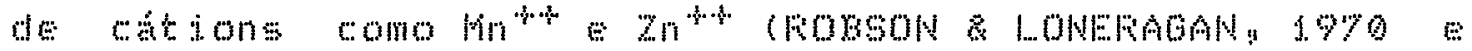

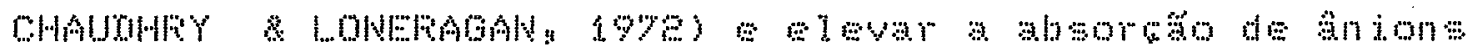

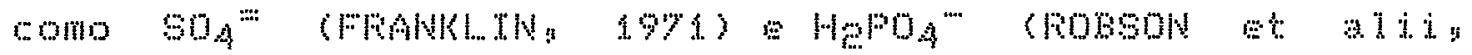
19\%0) entretanto pode afotar a encrada destes ions

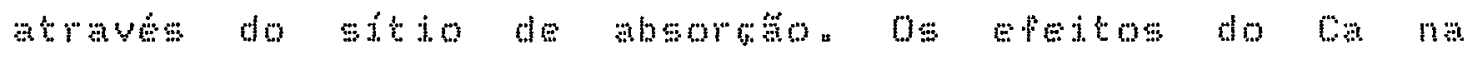

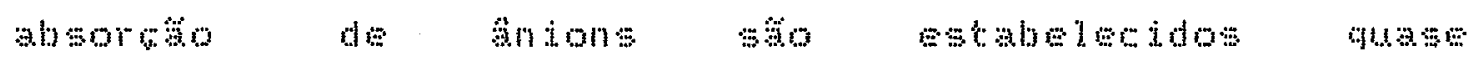

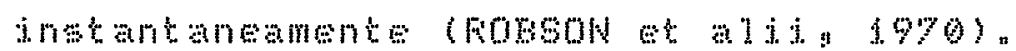

0 wito do ca nto soperticon cations trivalentes amontam a absorso de $\mathrm{HeO}_{4}$ mas que cations divalentes os quas term um mar efeito que chions

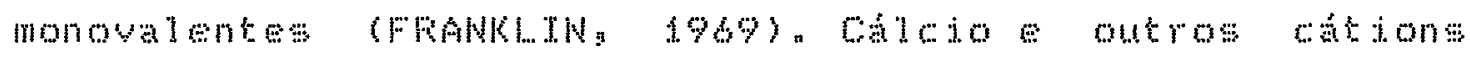
podm aumontar a abortio de andons do acordo com a dupla camada eletronica associada às corgas negativa fixas da parede olular e desta fom permite mator aceso polos 


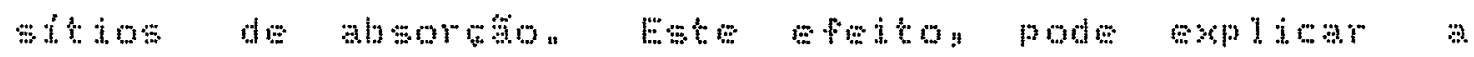

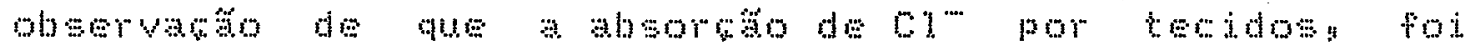

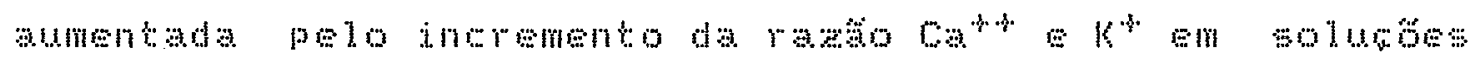

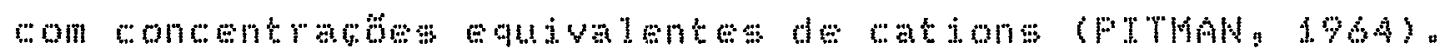
Aunento na conombrato externa de $k^{3}$ reduz

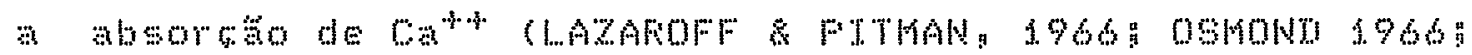

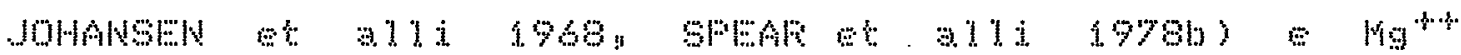

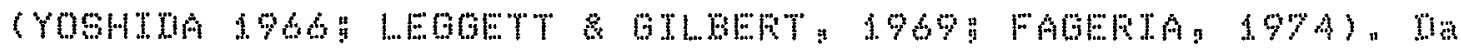
mesma forma otranspore do coth a longas distancias reduzido pela alta concentrabo de catons monovalentes

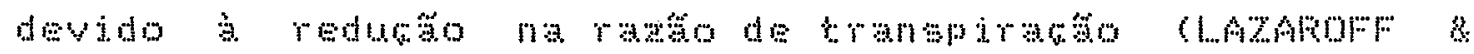
FTTMAN, 1966).

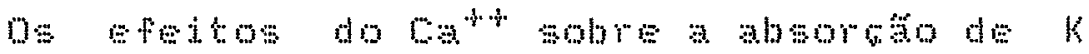

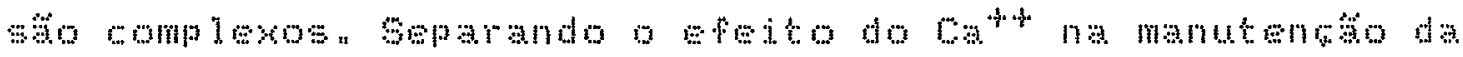
inteoridade da membrana afetando o aceso de ions aos

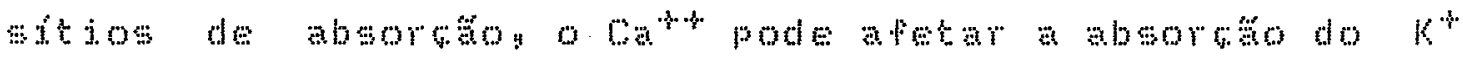

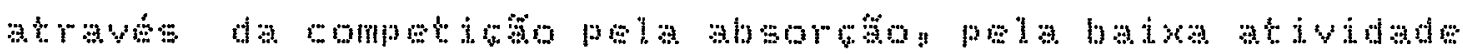

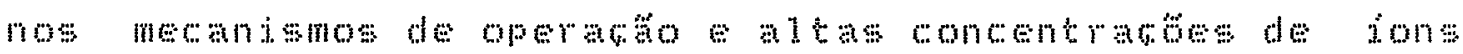
na $\operatorname{solug} 30$

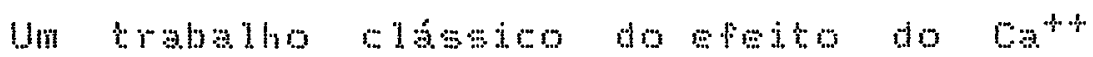

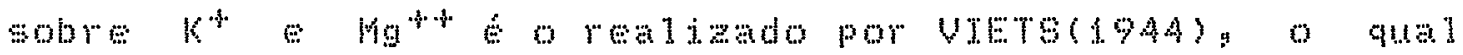

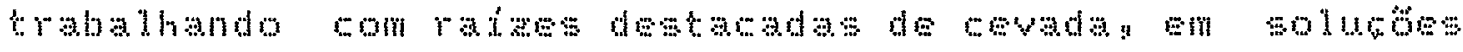
com vardacto na conentrato de ca de 0 a soomeal de

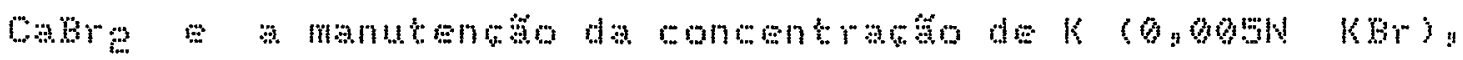
oborvou que em baxas concentraces de can as rases de

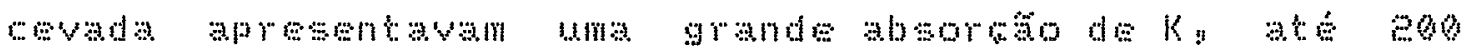

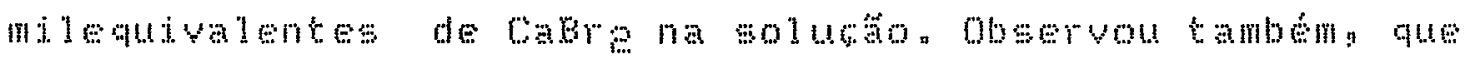




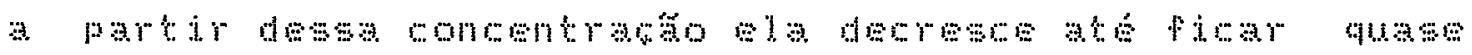
nula, quando a solucto atimge soo miloqualentes de caren 0 aumento do concentrato de come o eteito do opto

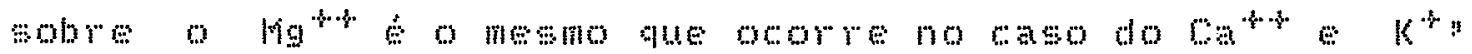
mostando um efeito signticativo ontre cat e mot mobro a aborato de K (MALAVOLTA: 1980). 


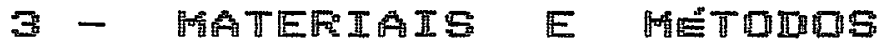

\subsection{Local}

0 ensaio foi inetalado no dia 10 do

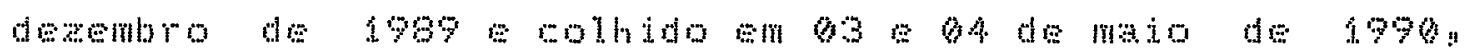
sendo condusido na casa de vegetacto do mepartamonto de

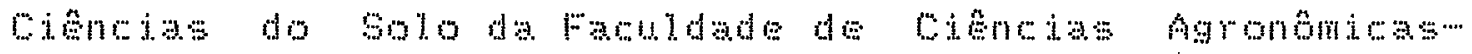
UNE:" "Campus" de Botucatu.

\subsection{Conducão do Ensaio}

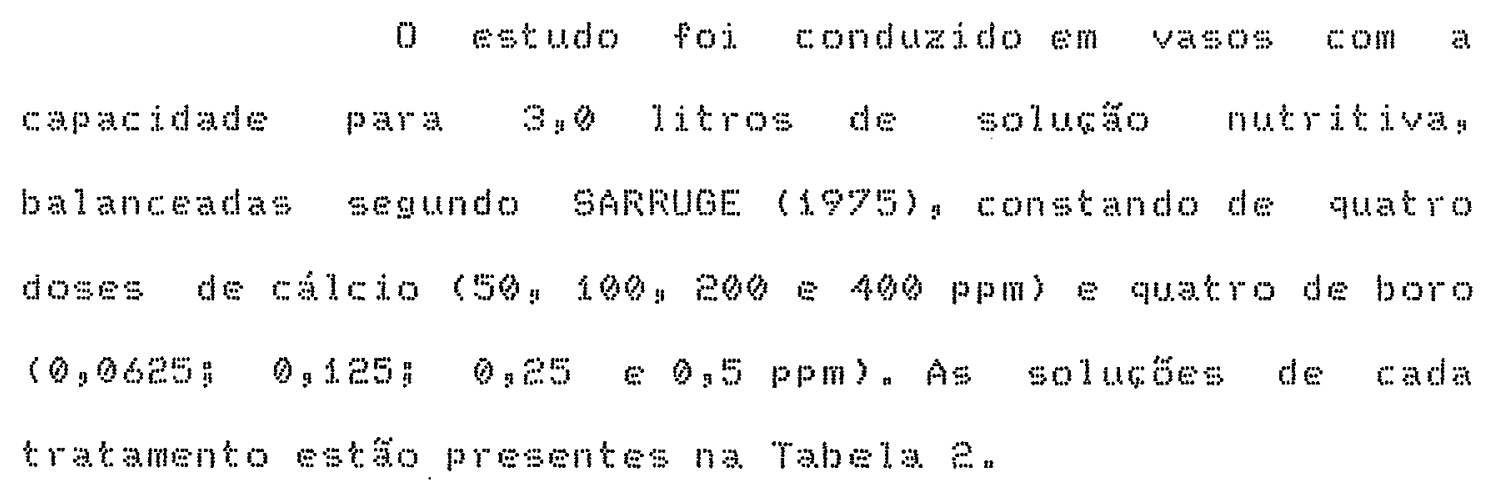


Tabela 2. Solucóes nutritivas utilizadas no trabalho, balanceadas segundo sarfube (1975)。

SOLUCA0 ESTOOUE (m1/1 de solugäo nutritiva)

\begin{tabular}{|c|c|c|c|c|c|c|c|c|c|}
\hline $\begin{array}{c}\text { Tratanentos } \\
\text { (ppm) }\end{array}$ & $\begin{array}{c}\mathrm{KH}_{2} \mathrm{PO}_{4} \\
\mathrm{H}\end{array}$ & $\begin{array}{c}\mathrm{KNO}_{3} \\
\mathrm{H}\end{array}$ & $\begin{array}{c}\mathrm{HgSO}_{4} \\
\mathrm{H}\end{array}$ & $\begin{array}{c}\mathrm{Ca}\left(\mathrm{WO}_{3}\right) \\
0.5 \mathrm{WH}\end{array}$ & $\begin{array}{c}\mathrm{HH}_{4} \mathrm{HIO}_{3} \\
\mathrm{O} .5 \mathrm{H}\end{array}$ & $\begin{array}{c}\mathrm{CaCl}_{\mathrm{E}} \\
\mathrm{H}\end{array}$ & $\begin{array}{l}\text { Solutato } \\
A^{*}(-B)\end{array}$ & $\begin{array}{l}\text { Solucăta } \\
\text { FeEDTA }\end{array}$ & 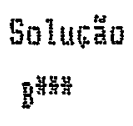 \\
\hline 50 & & & & & & & & & \\
\hline $8 \quad 0,0625$ & 1 & 5 & 2 & 2,5 & 7.5 & - & 1,0 & 1,0 & 9.5 \\
\hline $\mathrm{Ca} \quad 100$ & & & & & & & & & \\
\hline$\$ 0,0625$ & 1 & 5 & $\hat{E}$ & 5.0 & 5.0 & - & 1,3 & 1,0 & 3,5 \\
\hline Ca $\quad 200$ & & & & & & & & & \\
\hline $8 \quad 0,625$ & 1 & 5 & 2 & 10.6 & - & - & 1,6 & 1,0 & 6,5 \\
\hline $\mathrm{Ca} \quad 400$ & & & & & & & & & \\
\hline $\bar{i} \quad 0,0625$ & 1 & 5 & $\hat{z}$ & 10.0 & - & 5.0 & 1,0 & 1,0 & 0.5 \\
\hline 50 & & & & & & & & & \\
\hline B $\quad D_{125}$ & 1 & 5 & 2 & 2,5 & 7,5 & - & 1,6 & 1,0 & 1,0 \\
\hline $\mathrm{Ca} \quad 100$ & & & & & & & & & \\
\hline E $\quad 0.125$ & 1 & 5 & 2 & 59 & 5,0 & - & 1,9 & 1.0 & 1.90 \\
\hline $\mathrm{Ca} \quad 200$ & & & & & & & & & \\
\hline $5 \quad 0,125$ & 1 & 5 & 2 & 10,0 & - & - & 1,6 & 1.0 & 190 \\
\hline $\mathrm{C} \quad 490$ & & & & & & & & & \\
\hline В 0.125 & 1 & 5 & 2 & 10.0 & 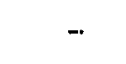 & 5.6 & 1.0 & 1.0 & $\frac{1}{2}, 0$ \\
\hline
\end{tabular}

continustöo. a 


\begin{tabular}{|c|c|c|c|c|c|c|c|c|c|}
\hline $\begin{array}{c}\text { Tratamentos } \\
\text { (ppm) }\end{array}$ & $\begin{array}{c}\mathrm{KH}_{2} \mathrm{PO}_{4} \\
\mathrm{H}\end{array}$ & $\begin{array}{c}\mathrm{KNO}_{3} \\
\mathrm{H}\end{array}$ & $\begin{array}{c}\mathrm{MgSO}_{4} \\
\mathrm{H}\end{array}$ & $\begin{array}{c}\mathrm{Ca}\left(\mathrm{NO}_{3}\right) \\
0.5 \mathrm{H}\end{array}$ & $\begin{array}{c}\mathrm{NH}_{4} \mathrm{NO}_{3} \\
0_{9} 5 \mathrm{H}^{4}\end{array}$ & $\mathrm{Cacle}_{H}$ & $\begin{array}{l}\text { Solu- } 200 \\
A^{*}(-B)\end{array}$ & $\begin{array}{l}\text { Solută० } \\
\text { FEEDTA }\end{array}$ & 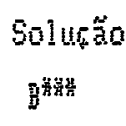 \\
\hline 50 & & & & & & & & & \\
\hline 0,25 & 1 & 5 & 2 & 2,5 & 7,5 & - & 190 & 1.0 & 29 \\
\hline $\mathrm{Ca} \quad 100$ & & & & & & & & & \\
\hline 9,25 & 1 & 5 & 2 & 5.0 & 5.0 & - & 1.6 & 1,0 & 2,0 \\
\hline 200 & & & & & & & & & \\
\hline 0,25 & 1 & 5 & 2 & 10,0 & - & - & 1,0 & 1,0 & 2,0 \\
\hline 400 & & & & & & & & & \\
\hline 0,25 & 1 & 5 & 2 & 10,9 & - & 5,9 & 1,6 & 190 & 2,4 \\
\hline 50 & & & & & & & & & \\
\hline 0,5 & 1 & 5 & 2 & 295 & 7,5 & - & 1,0 & 1.0 & 4.9 \\
\hline 100 & & & & & & & & & \\
\hline 0,5 & 1 & 5 & 2 & 5,0 & 5,0 & - & 1.0 & 1,0 & 4,0 \\
\hline 200 & & & & & & & & & \\
\hline 0.5 & 1 & 5 & $E$ & 10.0 & - & - & 1,0 & 1.0 & 4,0 \\
\hline 400 & & & & & & & & & \\
\hline 9.5 & 1 & 5 & 2 & 10.0 & - & 5,0 & 1,0 & 100 & 49 \\
\hline
\end{tabular}

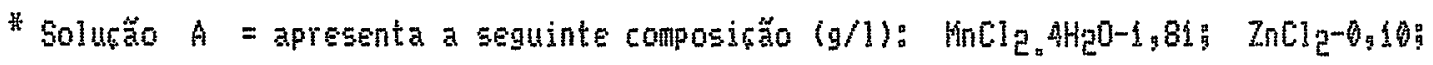

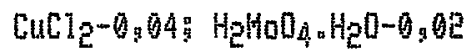

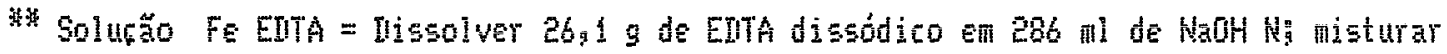

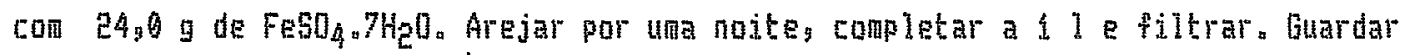
en frasco escuro tampado, efl geladeira.

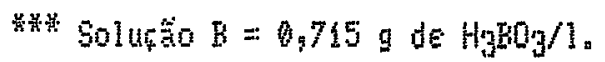




\title{
3.2.1. Material básico:
}

\begin{abstract}
A variedade porta enxerto utilizan foi o

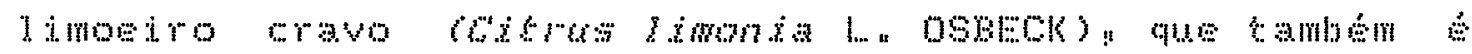
conhecido pelos nolles de "Franoss" "Bugre" "Rosa" e "Rangbur" (SAl. TEE 1978) sendo a variedade dominante na ejtricultura brasingara representando $90 \%$ dos porta

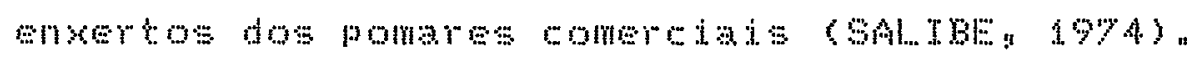

\subsubsection{Obtencão de Mudas.}

As mudes form rotradas do viveiro da

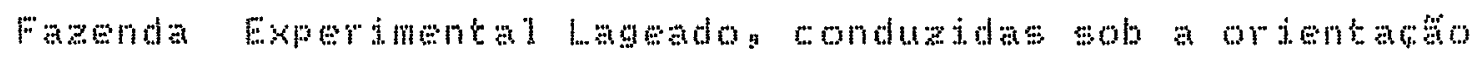

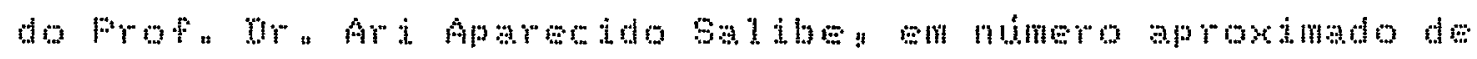
500 (quinhentas) gendo lavadas o separadas por dianetro

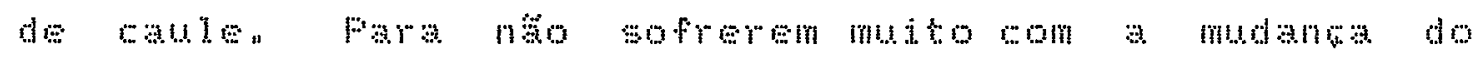
habitat as mudas passaran por un processo de aclimatooso indeiado 2 meses antes da instalaso do trabalno: como deserito a seguir"

$$
\begin{aligned}
& \text { a -.. } 3 \text { dias on Áma destiladag a sombra } \\
& \text { b } m \text { dias en solucto nutritiva de } \\
& \text { maronutrientes }(1 / 3 \text { da concentratosos a } \\
& \text { sombra. } \\
& \text { c } 10 \text { dias en solucio nutritiva de }
\end{aligned}
$$

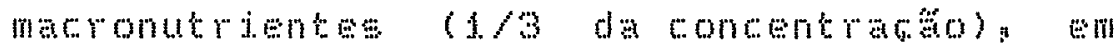


Cosa de Vegetaran

Apow estos 20 dias as modas sotreram poda

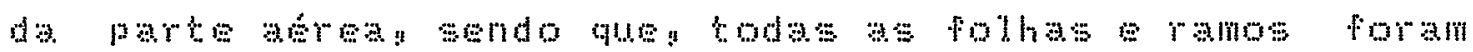
retirados permanecendo apenas o male prinapal

0 comprimento das rafose poj und forminado 15 cm 6 as plantas retomaran a casadewegetaconon prosesoundo o processo de ac limataction

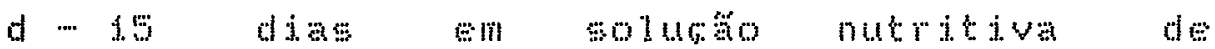

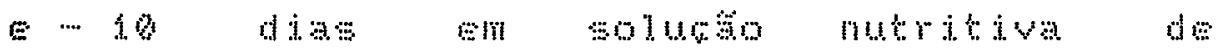

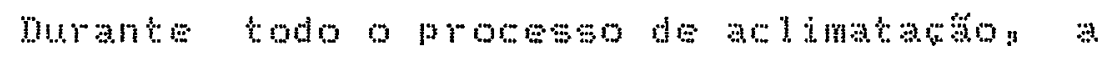

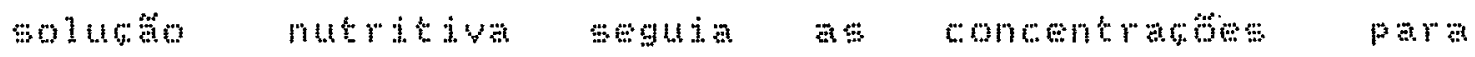

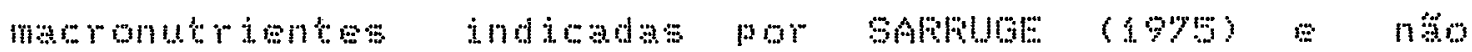
wontinhan os micronutrientes: para nởo arotar poster inmonte os tratamentos com doses menores de boro de solucto estoque lomposino dis soluga nutitiva de maconutrientes (mI de solug

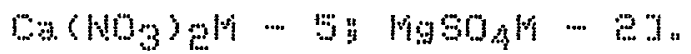




\subsubsection{Solucão nutritiva}

A solucro foj trocada a cada 15 dias gendo Peito acomanhamento semanal da conontratao dos

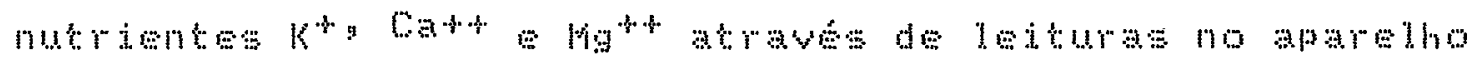
de absor

\subsection{Delineamento Experimental e Análise Estatistica.}

o delinemento exprimental foi o de blocos manaldzados com 16 tratamentos em comema patonal acraves das combinases de 4 niveis de caldo com a nivels

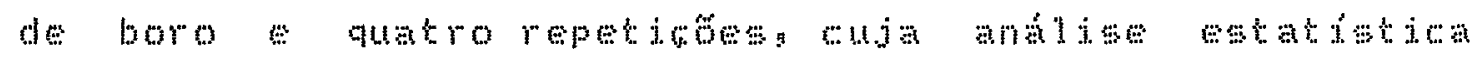

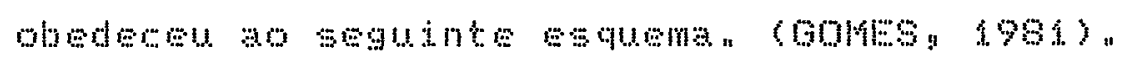

\begin{tabular}{|c|c|}
\hline Causa da Var iartió & $0.1 \ldots$, \\
\hline Bl ow & 63 \\
\hline Niveds de ca & 03 \\
\hline$W \cdot v=d \equiv d=G$ & 03 \\
\hline dntaracta ca $\times$ B & 09 \\
\hline Restuo & 46 \\
\hline Total & 63 \\
\hline
\end{tabular}




\begin{abstract}
A comparato entre as medias roalizadas pelo teste de Tukeyn sendo utiliando o programa estatstico denominado Extoon desenvoludo no peolo Computacional de Botucatu a.- Centro de Informatica na

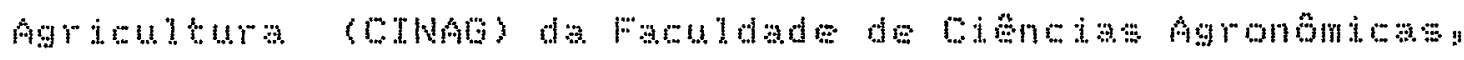
UNESF" "Campus" de Botucatu.
\end{abstract}

\title{
3.3.1. Tratamentos
}

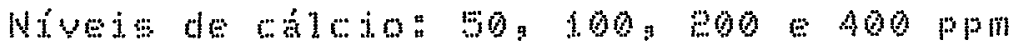

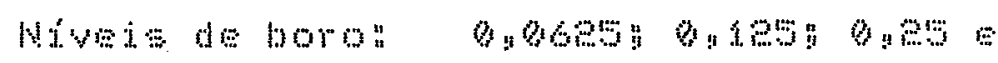

$$
\begin{aligned}
& 0, \Xi p: m
\end{aligned}
$$

\subsubsection{Parâmetros avaliados}

\subsubsection{Comprimento de raiz}

Para determinar o comprimento das ratas

utilizouse O apaTEIHO CORAIR FODTLENGTH SCANWER doado ao Departamento de cienejas do golo polo TARC ... Tropical

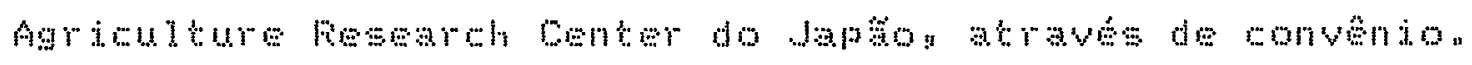
0 apamelho \& composto por um disco de

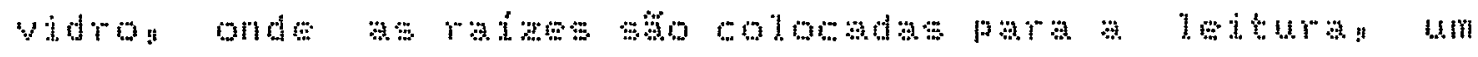

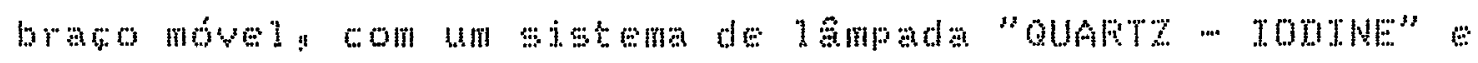


um senson diode do luminosidade e o comando do mparelho won lejtum digital (em metros) das amostras segundo igura in

o principio da leitura do comprimento de

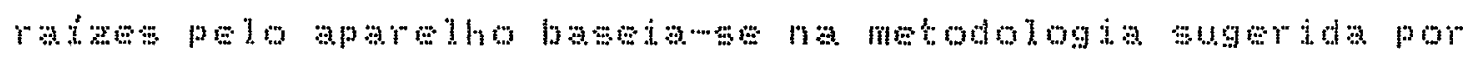
WEWMAN $(1968)$ atrase da formula:

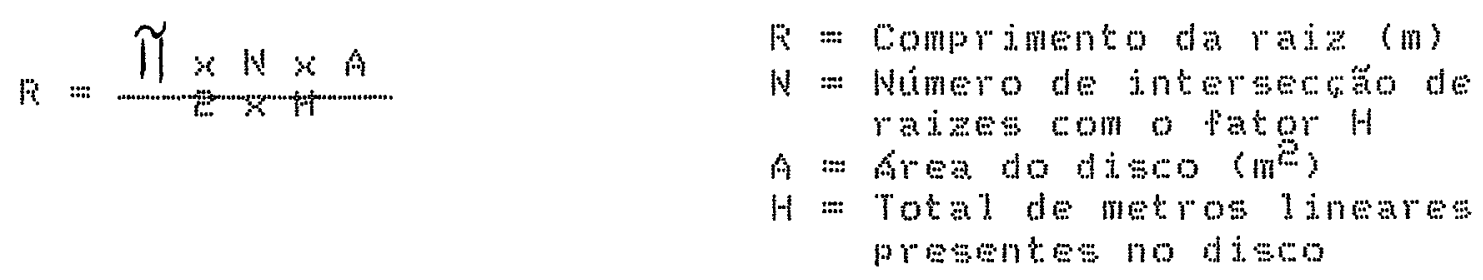

Fara se efetuar as loituras as rax devem ter un diamero menor que enm devem ser cortadas com 30 a 40 mm de comprimento devendo ser ben distribuidss sobre o discon

\subsubsection{Diâmetro do caule}

Medidas tomadas utiliandowse pagumetron obtias atraves da media de 4 medtas 5 cm acima do colo da plantag em nil inetron

\subsubsection{3. Área foliar e numero de foltas}

Fara decermarme a area foliar total de

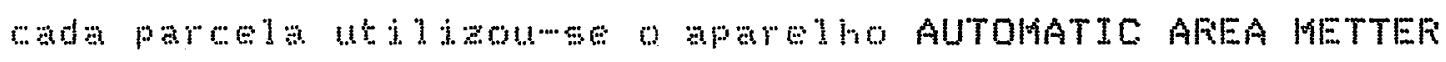




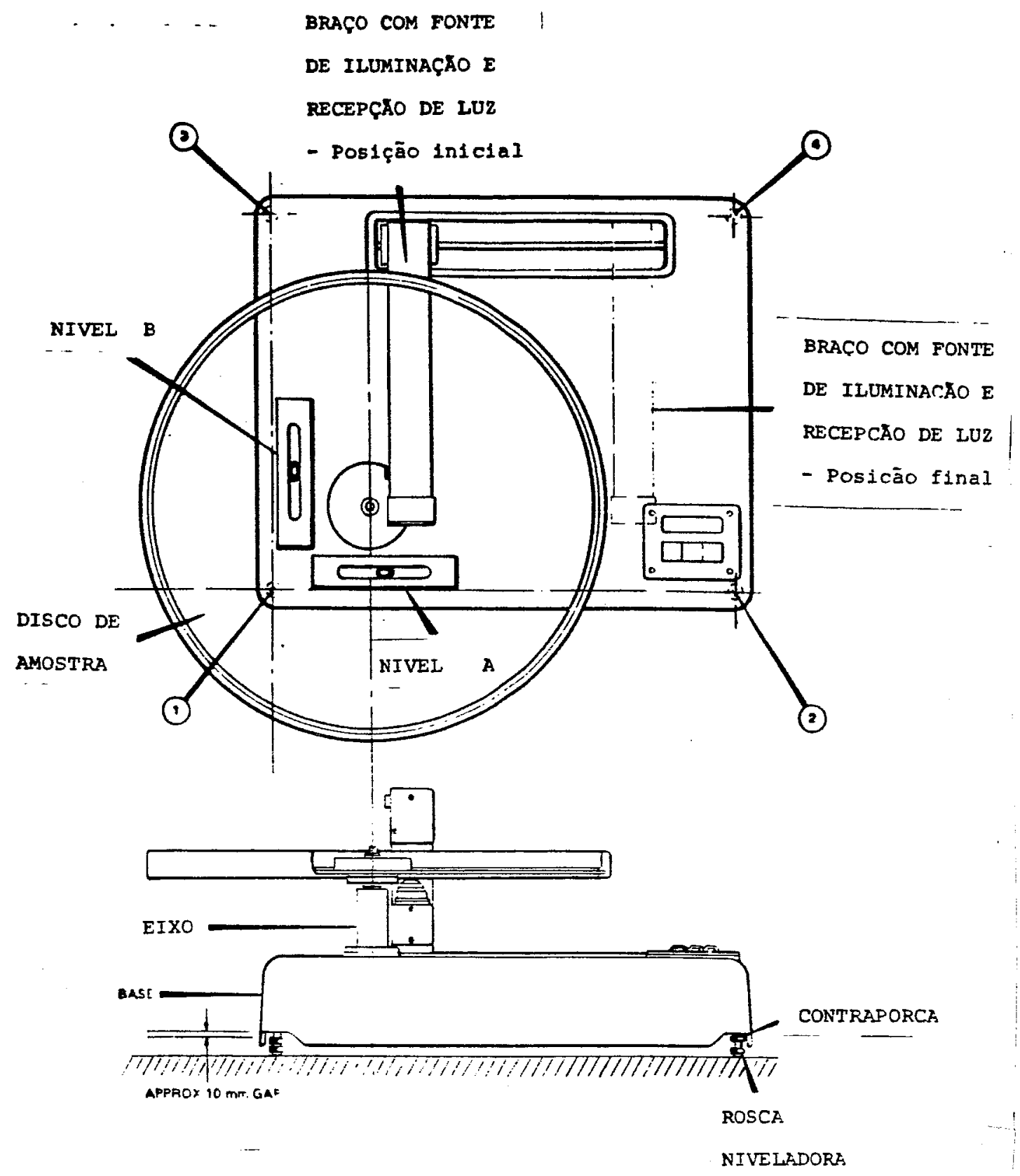

Figura 1: Esqema do aparelho Colman ROOTLENGTH SCANHER para medida de comprimento de raizes. 


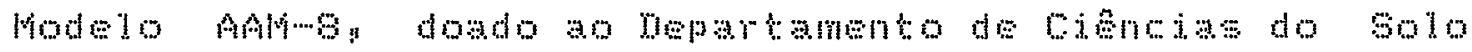

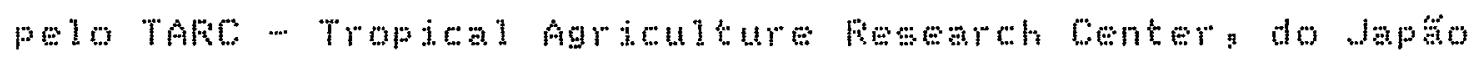

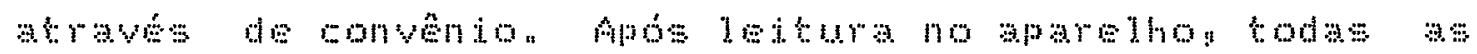
polhas poran contadas.

0 aparello e composto por uma undade

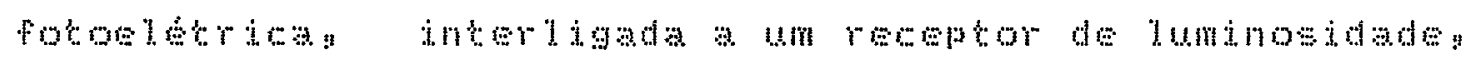

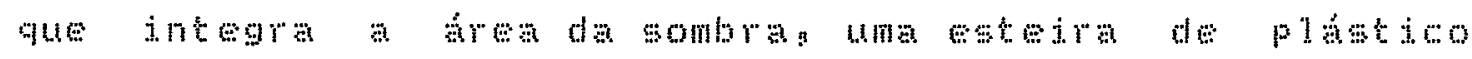
duplan de maneira que folla a ser determinada a area permaneca estendida e fixa a uma unidade de controle do

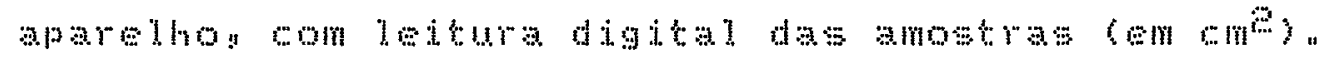

o principio de Funedonamento do apredto based

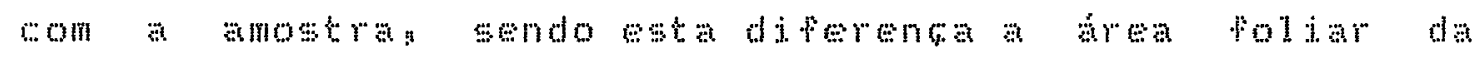
mosman

\subsubsection{Peso de matéria seca}

Fara se obtor o peso de metria secan o materjal vegetal toj colocado em estuta com circulaco Forada de ar a com fomperatura varando entre bs e 70 o ate peso constanten.

\subsubsection{Teores de nutrientes}

A concentrasaso de nutrientes nas ratess wale e Polhas fot deteminada atraves da mesodologia 


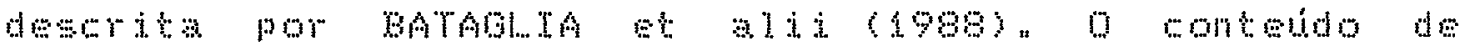
nutrientes poj alculado en funto do peso de materda seca

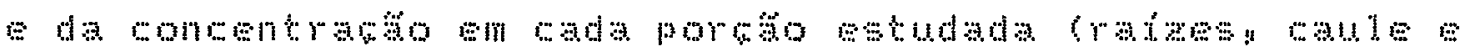
Polnas) 
A F FEULTAIDS E IIISLUSSRD

\subsection{Raizes}

\subsubsection{Peso de matéria seca e comprimento das raizes}

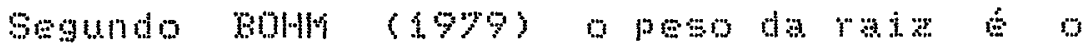
paranetro majs comum para o studo de rafas e que o peso da matria seco dove ser o parametro properido nividu

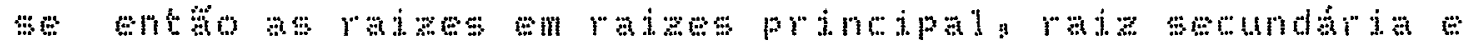
para determinato do peso de materia secan considerouses

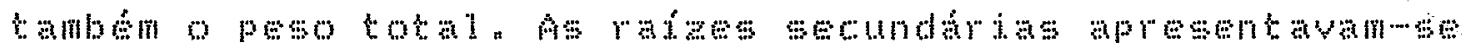
wom dismetro monor que emm em todos os tratamentos.

os resultados obtidos esta presentes nos

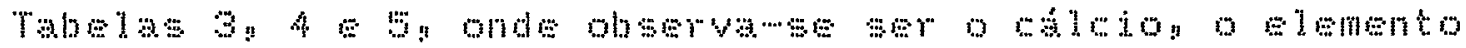
que mas aletou o desenvolvimento do istema radicular.

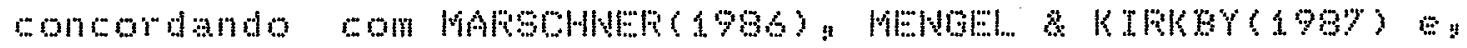
MALAVOLTA at ali(1989), e nâ havendo efejto

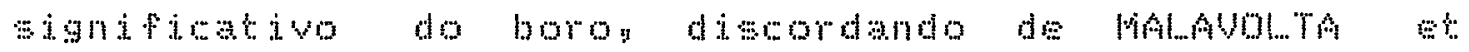

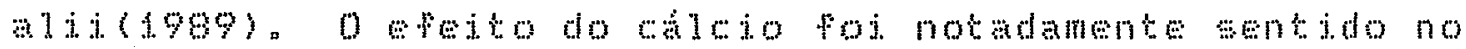

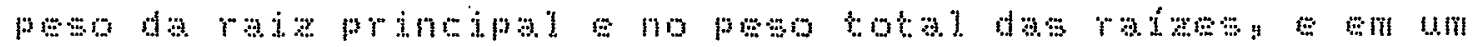

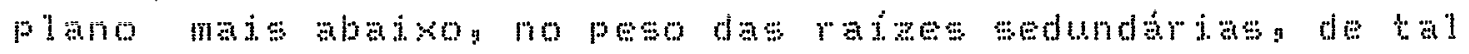
forman que os resultados nostram se segundo a ordem

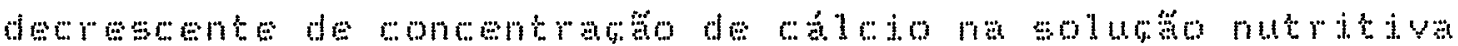

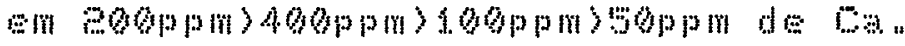




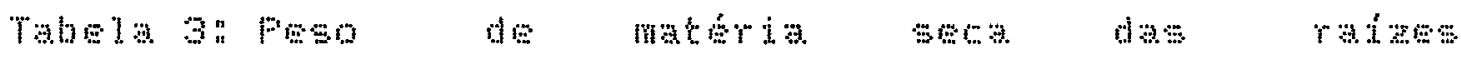
pincipara(giplanta) das plantas de limosimo mavo (media de 4 repeticose)

\begin{tabular}{|c|c|c|c|c|c|}
\hline & & & $\mathrm{Bppm}$ & & \\
\hline ap pam & $0,0<83$ & 0 , 128 & 0,23 & 0,5 & Media 6 \\
\hline 50 & $0,9 \%$ & 1,07 & 0,89 & 0,97 & $0,93 \%$ \\
\hline 100 & 1.00 & 1.00 & 0.91 & 0.96 & $0,99 \mathrm{C}$ \\
\hline eno & 1,70 & 1.63 & 1.69 & 1.65 & $1.69 \mathrm{~A}$ \\
\hline 400 & $1,1.1$ & 1,36 & 190 & 1,33 & $1,3 E \mathrm{~B}$ \\
\hline Media 8 & $\operatorname{sen} \pi$ & 1.28 & $x+35$ & $1: 18=$ & \\
\hline
\end{tabular}

* Hédias seguidas de letras maiusculas na vertical e minusculas na horizontal iguais năo diferem entre ei pelo teste de Tukey zo nivel de $5 \%$ de probabilidade.

Tabela 4 mes de materta seca de raizes secundarias(g/planta) das plantas de limoeiro mavo emedia de 4 repetiobes

\begin{tabular}{|c|c|c|c|c|c|}
\hline \multirow[b]{2}{*}{ cap pom } & \multicolumn{5}{|c|}{$\mathrm{B} p \mathrm{~mm}$} \\
\hline & $0,0,68$ & 0,125 & 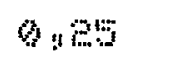 & 0,15 & Médin Ca \\
\hline$\because 0$ & 0,93 & 9,45 & $0,8 \%$ & $0,: 4$ & 0,00 \\
\hline 100 & 0.30 & 0,30 & 0 , & 0.20 & $0.28 \mathrm{~B}$ \\
\hline eno & $0: 5$ & $0,5 \%$ & $6,5 e$ & $0,: 34$ & $9,54 A$ \\
\hline 400 & 0,39 & 0.64 & 0,56 & 6,53 & $0,50 \mathrm{~A}$ \\
\hline Medjä & $6_{9} 40$ & 0,41 a & $\overline{0}, 40 \quad a$ & 0,39 & \\
\hline
\end{tabular}

* hedias seguides de letras maisculas na vertical e ninisculas no horizontal iguais năo di ferem entre si pelo teste de Tukay ao nivel de $5 \%$ de probabilidade. 


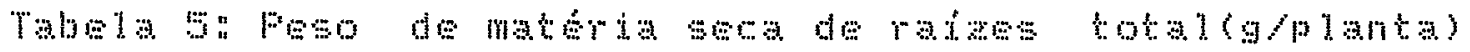
das plantas de limosiro cravo modia de

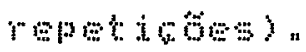

\begin{tabular}{|c|c|c|c|c|c|}
\hline \multirow[b]{2}{*}{ Capm } & \multicolumn{5}{|c|}{$\mathrm{g} \mathrm{pm}$} \\
\hline & 0,060 & $0,1 p$ & 0,85 & 0,3 & Medsm 6 \\
\hline 50 & $4: 31$ & 1,93 & 149 & 1,01 & $14: 1 \mathrm{c}$ \\
\hline 100 & $1: 3 x$ & $\sin 38$ & 1,16 & $\operatorname{sen} 4$ & $1,27 \mathrm{0}$ \\
\hline 200 & 2,36 & $2: 17$ & $2: 100$ & $2: 19$ & $2,83 \mathrm{~A}$ \\
\hline 400 & 1,90 & 1.90 & 2.06 & 1.87 & $1: 83$ A \\
\hline Media & 1 $461 \mathrm{a}$ & $1.696 a$ & $1.644 \pi$ & 1. 978a & \\
\hline
\end{tabular}

* Hédias seguidas de letras maiusculas na vertical e minúculas na horizontal iglais não diferem entre si pelo teste de Tukey ao nivel de $5 \%$ de probabilidade.

Fara realiar as medidas de comprimento de

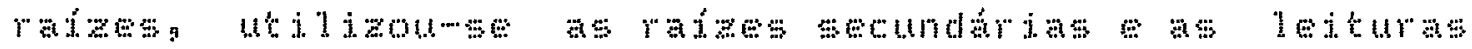

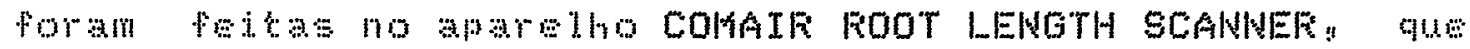
exige radzs: com tifnetro menor que emm 0s resultados achanse no Tabela ba onde observase que houve efejto do

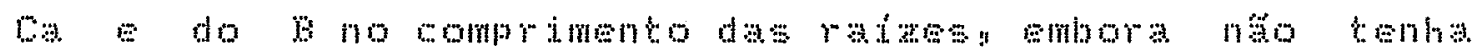
havido jnteraço significativa entre eles. A medida que se Elovo a conontraso de ca na solusto nutritivan observouse aumento no comprimento sendo que podemos

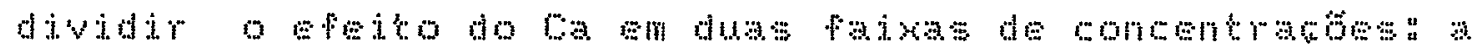
primeiras que womprende as monentrabes de 50 e $100 \mathrm{ppm}$ de ca y que apresentaram comprimono modio de ratzos de

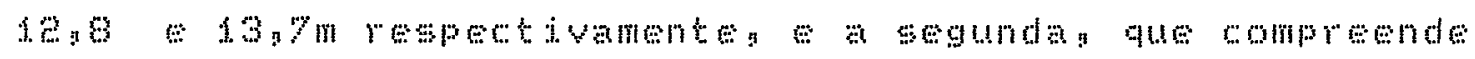

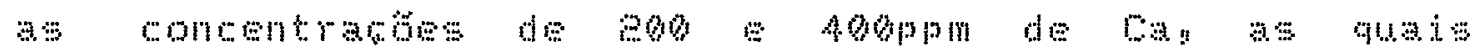




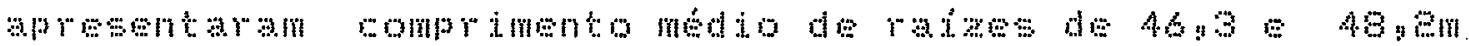
resperturamenten representando un aumento de 3 g veres no comprimento de raiza da primeira para a segunda fadxa de concentratso.

Tabeda ba comprimento das razes (mplanta) das plantas du

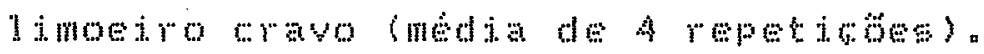

\begin{tabular}{|c|c|c|c|c|c|}
\hline \multirow[b]{2}{*}{ \% } & \multicolumn{5}{|c|}{$\mathrm{B} p \mathrm{pm}$} \\
\hline & $0,0 \sin$ & $0,11,3$ & $0,8: 3$ & 0,5 & Média Ca \\
\hline 50 & $18: 97$ & 11960 & 13,80 & $1.3: 10$ & $12,8 \%$ \\
\hline 1. ho & $1+4: 4$ & $13: 6 \%$ & 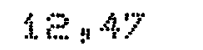 & $14: 30$ & 13,768 \\
\hline 200 & 38.98 & $43: 97$ & 49,75 & 92,90 & $46.36 \mathrm{~A}$ \\
\hline 400 & $34: 30$ & $\because 2: 62$ & 56 , 80 & $53,4 \%$ & $48: 00 \mathrm{~A}$ \\
\hline 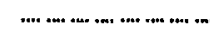 & - & 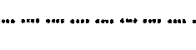 & | & 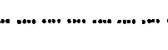 & . \\
\hline Modia & $25,130 \mathrm{~b}$ & $30,450 b$ & $30,89 \mathrm{ab}$ & $33,3 \%$ & \\
\hline
\end{tabular}

* Médias seguidas de letras mausculas na vertical e minúculas na horizontal iguais näo diferem entre si pelo teste de Tukey an nivel de $5 \%$ de probabilidade.

Com relatio ao 8 , os resultados noto foram

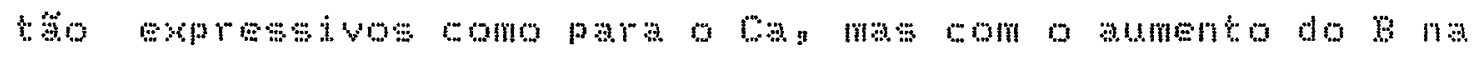
solumo nutritiva houve aumento no cresedmento da raiza de modo que os resultados apresentamsen em functo da

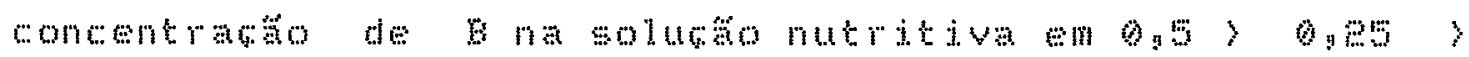

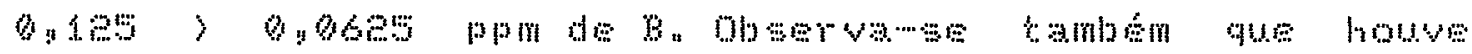

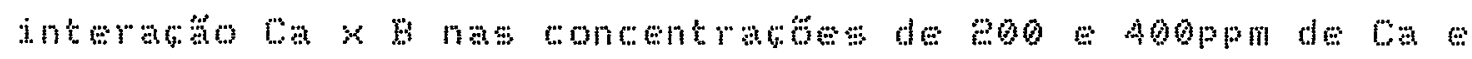

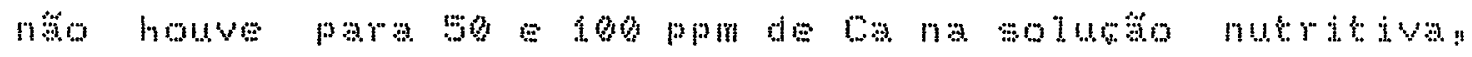
sendo que este dicino aso deve estar relacionado a uma postwel depiciencia de cáledo na plante podendo se supor 


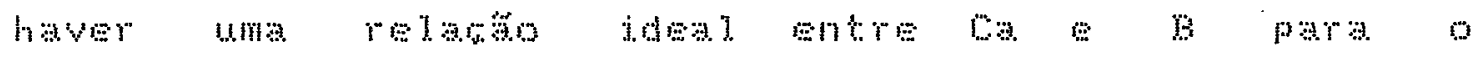
desenvolvimento do sistema radicular. Nânoi encontrado

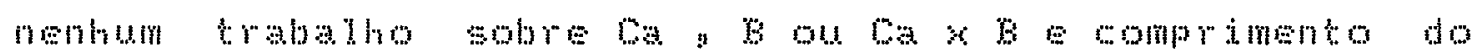
s.stema radicular gu outro nutriente esencial.

\subsubsection{Concentracão e acúmulo de nutrientes pelas raizes}

Welos resultados encontrados notames un comportamento diferenciado entre os moronutrientes de tal forma que visual dza-me tres ojtuabes distintas.

Fara Na pes observase que a modia que se aumenta a concentragto de Ca diminue a concentracoso desses elementos nos teotos das ratas conforme mostram as Tabolas 7,9 a is respetivamentes sendo este fato

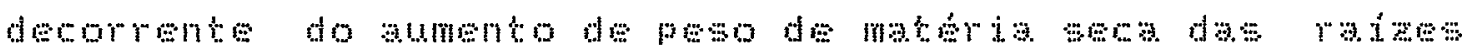
cusado pelo aumento da conoentrato de cas ocomondo

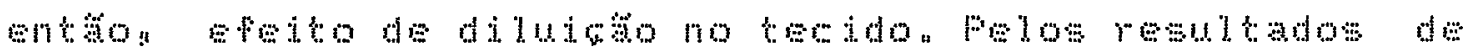
acumulo de N, Fe 5 apresentados nas Tabelas

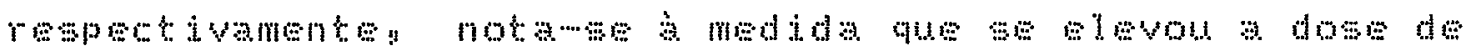
Qa aunentou o actmalo desses elementos ate eooppm de can havendo uma queda no acimulo quando passouse para tooppm de ca na soluga. Tal efeito e comprovado por

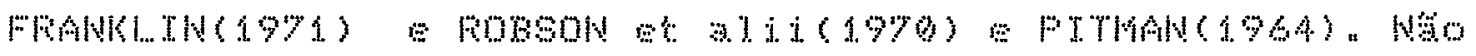
houve efejto das doses de boro ma conentratio ou ro

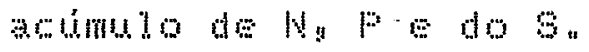




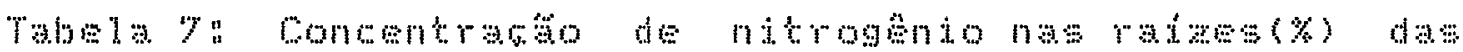
plantas de limoeiro mravo cmedia de 4 repetis $6 \pi s$

\begin{tabular}{|c|c|c|c|c|c|}
\hline \multirow[b]{2}{*}{ Ca ppm } & \multicolumn{5}{|c|}{ \& ppri } \\
\hline & $0,062 \%$ & $9,1,20$ & 0,83 & 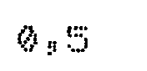 & Media Ca \\
\hline 50 & 1,40 & 1. : $1: 8$ & $1,3 e$ & 1. $: 4: 6$ & $1,48 \mathrm{~A}$ \\
\hline 100 & 1.63 & $\sin 3$ & 1,47 & 1,30 & 19449 \\
\hline eop & $4: 96$ & $1: 01$ & 0,96 & 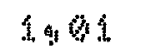 & $190 \mathrm{~g}$ \\
\hline 400 & 1.04 & 1.05 & 0.98 & 1.06 & $1.03 \mathrm{AB}$ \\
\hline Media & 1 : 29 & I : $24 \pi$ & 1. $18 \mathrm{a}$ & $x: 24 a$ & \\
\hline
\end{tabular}

* Médias seguidas de letras maiusculas na vertical e mintisculas ne horizontal iquais năo diferem entre si pelo teste de Tukey ao nivel de $5 \%$ de probabilidade.

Tabela 8" Acumulo de nibrogento nas ratese(maplanta) das plantas de linoejro cravo kitadia de 4 repetis $60 \%$;

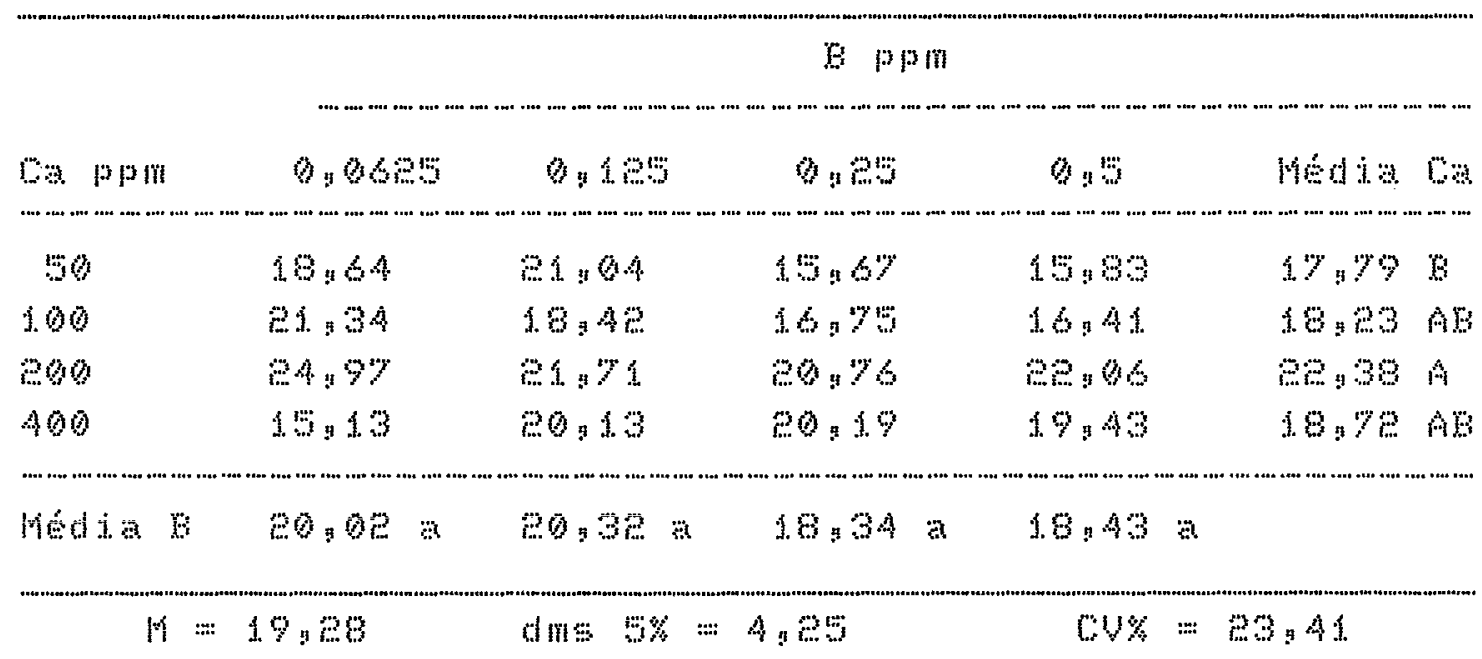

* hédias seguidas de letras maisculas na vertical a minusculas na horizontal iguais năo diferen entre si pelo teste de Tukey ao nivel de sy de probabilidade. 


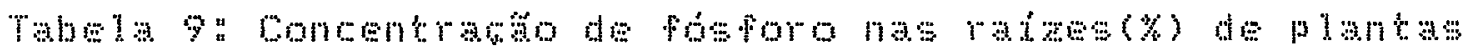
de limoeiro cravo (media de 4 repetictes).

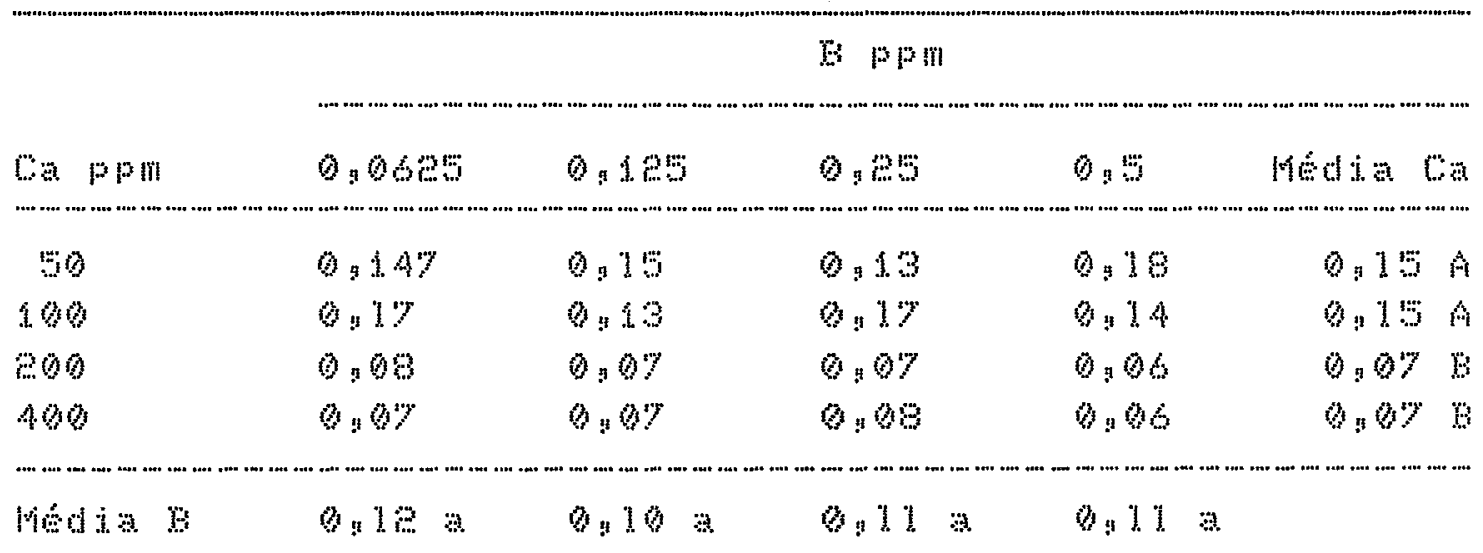

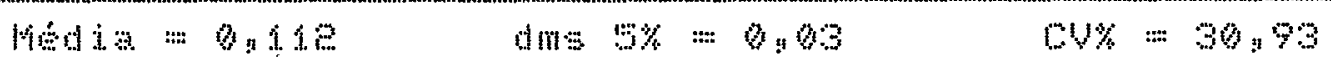

* hediss seguidas de letras maiusculas na vertical e minisculas na horizontal iguais nắ diferem entre 5 i pelo teste de Tukey an nivel de $5 \%$ de probabilidade.

Tabela to: Actmulo de fostoro nas ratzesolplanta) das plantas do limosiro cravo bodia do Tepetisess?

\begin{tabular}{|c|c|c|c|c|c|}
\hline \multirow[b]{2}{*}{ \% ppm } & \multicolumn{5}{|c|}{$8 \mathrm{ppm}$} \\
\hline & $0,0,083$ & $0, x+3$ & 0,28 & 0,9 & Modiz \\
\hline 50 & a $y 2$ & $1, \% 7$ & 1956 & 1,80 & $1: \% \mathrm{AB}$ \\
\hline 100 & e.e & 1,79 & $1.6 \%$ & 1.68 & $1.89 A$ \\
\hline 200 & $2: 08$ & $1: 15$ & 1.56 & $1: 41$ & $1: 64 \mathrm{AB}$ \\
\hline 400 & $1: 01$ & $1: 41$ & 1.62 & $1: 2 e$ & 1,318 \\
\hline Nedia 8 & 1476 & $1.6 \%$ & $1.60=$ & $1 \% 3$ & \\
\hline
\end{tabular}

* Médias seguidas de letras maiduculas na vertical e minuscuias na horizontal iguais năo diferen entre si pelo teste de Tukej ao nivel de 5 to probabilidade. 


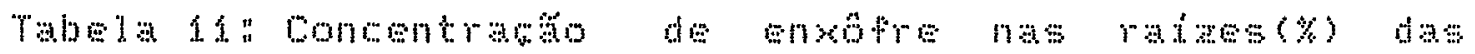
plantas de limotio cravo lmedia de 4 $r \cos i \sin s$

\begin{tabular}{|c|c|c|c|c|c|}
\hline \multirow[b]{2}{*}{ Sappla } & \multicolumn{5}{|c|}{$\mathrm{Bpm}$} \\
\hline & $0,0,68$ & 0,12 & 0,85 & $0: 3$ & Media \\
\hline 80 & 0,09 & 0,10 & $0: 09$ & 0,10 & $0,10 \mathrm{~A}$ \\
\hline 100 & 0,10 & $0,0 \%$ & 0,09 & 0,08 & $0.09 \mathrm{~A}$ \\
\hline 800 & 0,06 & $0: 06$ & 0.06 & 0,07 & $4: 46 \mathrm{~B}$ \\
\hline 400 & 0,06 & 0.06 & 0.07 & 0,07 & $0.07 \mathrm{~B}$ \\
\hline
\end{tabular}

Meda $=0,070$ dms $5 \%=0,01 \quad$ cU\% $=18,80$

* Hedias seguidas de letras oadusculas na vertical a minúsculas na horizontal iguais nầo diferen entre ei pelo teste de Tukey ao nivel de 5 de probabilidade.

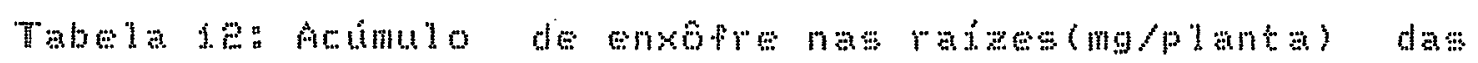
plantas do limosiro cravo lmodia do 4 repetsosen

\begin{tabular}{|c|c|c|c|c|c|}
\hline \multirow[b]{2}{*}{ Capm } & \multicolumn{5}{|c|}{ B ppm } \\
\hline & $0,06 e^{\circ}$ & 0,128 & 0.8 & 0,15 & Medin $\mathrm{C}$ \\
\hline 50 & $1, \geqslant 1$ & 1.30 & 1,00 & 0.99 & 1.414 \\
\hline 100 & 1,29 & 1. 13 & 1.01 & 0,98 & 1. 1.08 \\
\hline $80 \%$ & 19 & $1: 41$ & $1: 39$ & $1: 43$ & $1: 43 \mathrm{~A}$ \\
\hline 400 & 0,85 & $1: 16$ & 1.49 & $1: 36$ & $1, \ldots A B$ \\
\hline
\end{tabular}

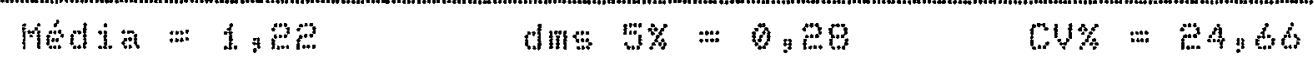

* Medias seguidas de letras aaiusculas na vertical g rinusculas na horizontal iguais nầ diferen entre si pelo teste de Tukey ao nivel de 5 de probabilidade. 
Fara K e Ma: nto houve efeito do be ou do $\mathrm{k}$ sobre a concentracto destes elementos nos toudos das

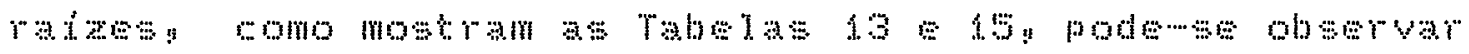
pelas Tabelas it e jos que ate a concentrato de eooppm na

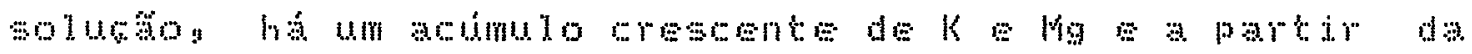

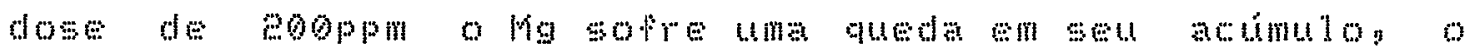

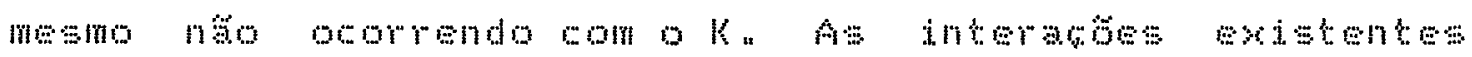

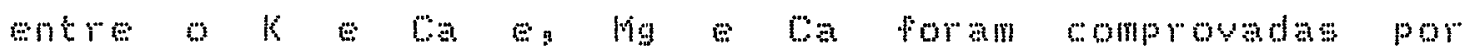

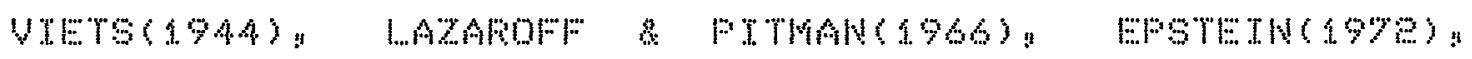
MALAUOLTA(1980!, MABSCHNER(A986) E MINOEL \& KTRKOY(1987): nio havendo efoto das doses de noro na conentrasos no acumulo de $K$ e Ms.

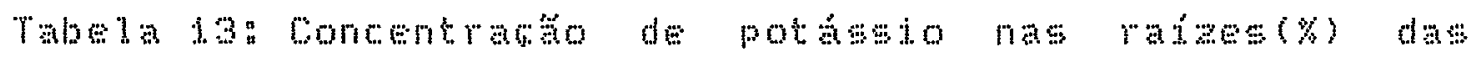
plantas do limosiro cravo medias de repetises;

\section{E prm}

\begin{tabular}{|c|c|c|c|c|c|}
\hline $\mathrm{ppm}$ & 0,0625 & 0,5 & 0,85 & 0,5 & Menda क \\
\hline 50 & 0,37 & 0,47 & 0,37 & 0,45 & $0: 4 . A$ \\
\hline 100 & 0,51 & 0,87 & 0,95 & 0,30 & $0.36 \mathrm{~A}$ \\
\hline eno & 0,50 & $0,3 \%$ & $0: 40$ & $9: 41$ & $0,42 \mathrm{~A}$ \\
\hline 400 & 0.43 & 0,39 & 0,43 & 0,42 & $0,4 \mathrm{EA}$ \\
\hline Medin & $0,48 \pi$ & $0,30 \mathrm{a}$ & $0.38 a$ & 0.390 & \\
\hline
\end{tabular}

* Hédias seguidas de letras maitisculas na vertical e minúsculas na horizontal iguais näo diferem entre si pelo teste de Tukey ao nivel de 5 de probabilidade. 


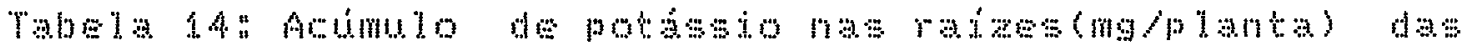
plantas de limosito cravo cmedias de 4

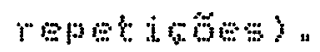

\section{B ppin}

\begin{tabular}{|c|c|c|c|c|c|}
\hline$\infty \mathrm{ppm}$ & $0,0,6 e$ & 0,185 & 0,8 & $0: 5$ & Meda \\
\hline 50 & 4,87 & 6,33 & $6 ! 97$ & 5.62 & 5.778 \\
\hline 100 & $6: 07$ & 3,84 & 3,42 & 3,18 & $4: 10 \mathrm{C}$ \\
\hline 200 & $10,0 \mathrm{~A}$ & 633 & 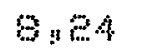 & 7,69 & $8,98 \mathrm{~A}$ \\
\hline 400 & 6,37 & 7.99 & 8.96 & $9.4 \%$ & $8,18 A$ \\
\hline Medan 6 :: & 6989 & t. $18 \mathrm{a}$ & 6,78 & $6,46 n$ & \\
\hline
\end{tabular}

* Médas seguidas de letras maísculas na vertical e ninúsculas na horizontal iguais no diferell entre si pelo teste de Tuky ao nivel de su de probabilidade.

Tabela 15 " Conmentrato do monssio nas ratzos\% das plantas de 1 inoejro eravo cmedia de 4

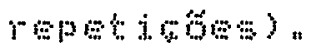

\begin{tabular}{|c|c|c|c|c|c|}
\hline \multirow[b]{2}{*}{ Ca ppm } & \multicolumn{5}{|c|}{$\mathrm{B}$} \\
\hline & 0,0605 & 0,125 & 9,83 & $a, a$ & Modin Ca \\
\hline 50 & 0,04 & $a_{9}, 9$ & 0,04 & $b_{11}, 4$ & $0,94 A$ \\
\hline 100 & 0.04 & 0.03 & 0,04 & 0,04 & $0,04 \mathrm{~A}$ \\
\hline e00 & $0,0,3$ & $0: 04$ & 0,04 & $\theta: 4 n$ & $0,04 \mathrm{~A}$ \\
\hline 400 & 0,04 & 0.03 & 0,03 & 0,03 & $0,04 \mathrm{~A}$ \\
\hline mota & $0,0, \quad a$ & $0,04 \quad a$ & $0.04 \quad 3$ & 0,04 & \\
\hline
\end{tabular}

* Hédies gegudas de letras maiusculas na vertical e minusculas na horizontal iquais nowo diferem entre si pelo teste de Tukey ao nivel de 5 de probabilidade. 


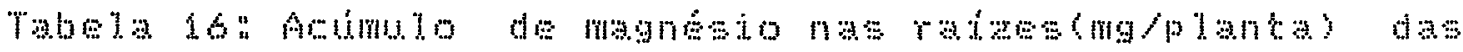
plantas de limosiro cravo biédia de $A$ repotiosis?

\begin{tabular}{|c|c|c|c|c|c|}
\hline \multirow[b]{2}{*}{ Cop pam } & \multicolumn{5}{|c|}{ B prom } \\
\hline & 0,060 & $0,5,83$ & $0,2 \pm$ & 0,5 & Hodin \\
\hline 50 & 9,5 & $0: 33$ & 0,31 & $0,4=$ & $0: 30 \mathrm{~B}$ \\
\hline 100 & 0.54 & $0,: 1$ & 0,48 & 0,47 & 0,508 \\
\hline 200 & 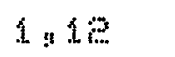 & 0,83 & 0,93 & 0,98 & $9.96 \mathrm{~A}$ \\
\hline 400 & 0,61 & 0,67 & 0,72 & 0,66 & $0.66 \quad 9$ \\
\hline Media 6 & 0,703 & $0.64 a$ & $6.66 n$ & 6,64 & \\
\hline
\end{tabular}

$$
\text { Média }=0,66 \quad \text { dns } 6 \%:=0,19 \quad 04 \%=34,3 \%
$$

* Hédias segudas de letres masusculas na vertical e minusculas na horizontal iguais nao diferem entre si pelo teste de Tukey ao nivel de 5\% de probabilidade.

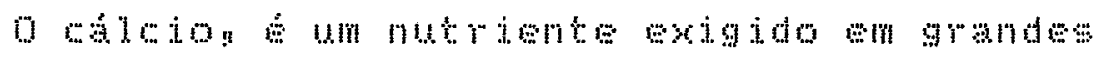

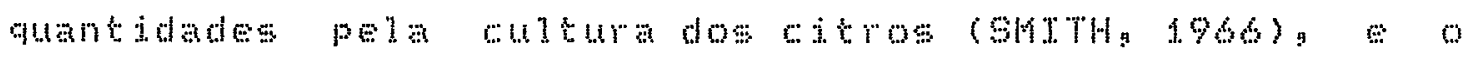
aunento de su teor na solusto nutritiva resteou wa aumento wa seu teor no tecido vegetal como mostra a Tabela

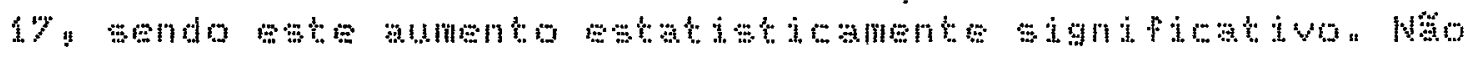
houve efejto de dose de sobre o teor de ca na raza ouanto ao acimulo de ou o comporamento fod o mesmo da

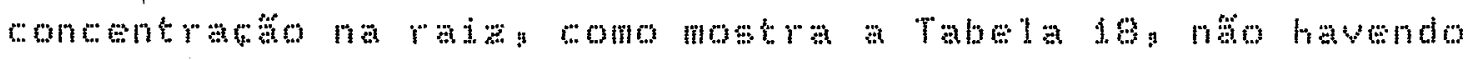

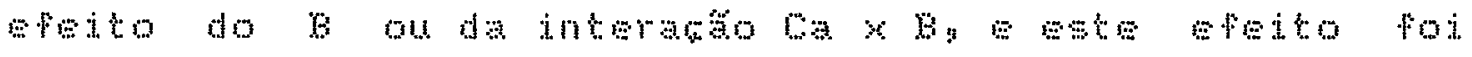

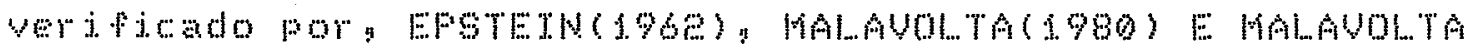
w a di $(1989)$ "

malisandowe o 8 nas ratess podewse

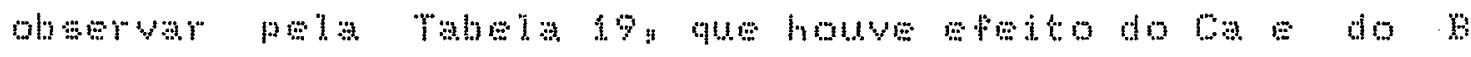
sobre a concentrato de 8 no terido de forma que 


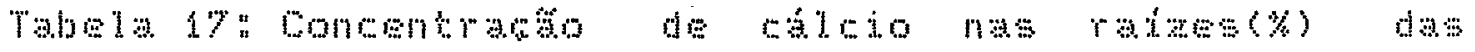
plantas de linoejro cravo cmedias de 4 repetioses?

\begin{tabular}{|c|c|c|c|c|c|}
\hline & & & в ррп & & \\
\hline Capm & 9,960 & $a+28$ & $a, 83$ & $\theta, 6$ & Meda 8 \\
\hline 30 & $0, \infty$ & 0,4 & 0,04 & $0,8 y$ & $0,25 \mathrm{c}$ \\
\hline 100 & 0,30 & 0,24 & 0,30 & $0,: 99$ & $0,28 \mathrm{C}$ \\
\hline eno & $a, 5: 3$ & $0: 54$ & $9: 54$ & 0,51 & $B ! 3 \quad \mathrm{~B}$ \\
\hline 400 & 0,57 & 0.64 & 0,71 & 0.57 & $0.62 \mathrm{~A}$ \\
\hline Media & $0_{0} 41 \quad a$ & $0,48 \quad n$ & $0: 45 \quad \mathrm{a}$ & 0,41 & \\
\hline
\end{tabular}

* Hédias seguidas de letras maiúsculas na vertical e minuscules na horizontal iguais năo diferem entre si pelo teste de Tukey ao nivel de $5 *$ de probabilidade.

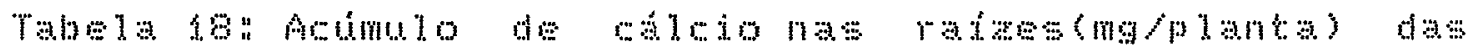
plantas de limosiro cravo medias de 4 repetis $5 \%=$

\begin{tabular}{|c|c|c|c|c|c|}
\hline \multirow[b]{2}{*}{ Ca ppin } & \multicolumn{5}{|c|}{ G pin } \\
\hline & 9,0625 & $9,18 \%$ & 0,3 & 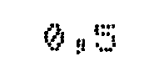 & Med dis Ca \\
\hline 50 & 3,00 & 3,23 & 2,00 & 247 & 2,969 \\
\hline 100 & 3,91 & 3,30 & $3: 42$ & $3: 66$ & $3,59 \mathrm{~B}$ \\
\hline 200 & 13,31 & 1) 959 & $11: 96$ & A 1 : $1 \mathrm{~A}$ & $1199 \mathrm{~A}$ \\
\hline 400 & 8,12 & $12: 41$ & $14: 66$ & 10,74 & $11: 48$ म \\
\hline 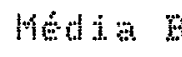 & $7,60 \mathrm{n}$ & 7,639 & 8,213 & $7: 06 a$ & \\
\hline
\end{tabular}

Media :-: 7,50

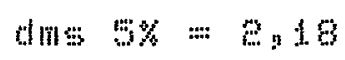

$\mathrm{CU}=30,85$

* Hedias seguidas de letras maibcules na vertical e minusculas na horizontal iguas năo diferem entre si pelo teste de Tukey an nivel de 5 de probabilidade. 


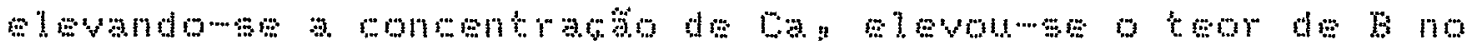

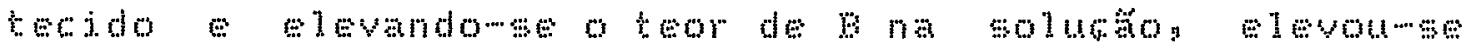
tamber o a no teodon mas no houve eferto da interaso Caxb. Fra o acumulo de 8 na rajag há apenas o efeito de

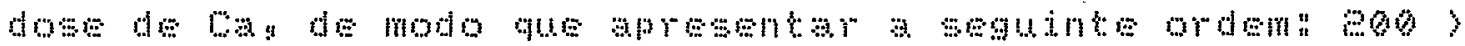
$400>100>50$, como mostra a Tabela eo.

Tabela hy concentrato de boro nas ratase(pmo das plantas de limosimo movo medias de 4 reperisos?

\begin{tabular}{|c|c|c|c|c|c|}
\hline \multirow{2}{*}{ Gар ppm } & \multicolumn{5}{|c|}{ Eppm } \\
\hline & 0,060 & 6,185 & 0,5 & 0,9 & Media m \\
\hline 160 & 60.78 & 10,80 & $18: 73$ & $21: 90$ & $19: 75 \mathrm{AB}$ \\
\hline 100 & 19.20 & 20,75 & $19: 26$ & $17: 30$ & $19.18 \mathrm{~B}$ \\
\hline 800 & 17,70 & 28,40 & 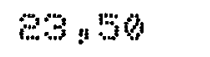 & $28: 7$ & $\mathrm{EQ}, 00 \mathrm{~A}$ \\
\hline 400 & 17,00 & $19: 0$ & $23: 50$ & 27,00 & ㅅ. $75 \mathrm{AB}$ \\
\hline Media $\mathrm{B}$ & 19.180 & $0,18 a b$ & $21,25 a b$ & $28 \div 66$ & \\
\hline
\end{tabular}

* Hédias seguidas de letras maisculas na vertical a minúsculas na horizontal iguais nă diferen entre si pelo teste de Tukg ao nivel de 5th de probabilidade. 


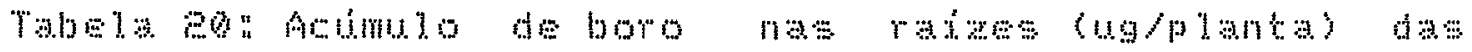
plantas de limosiro cravo credias de 4 $r \operatorname{sect} i \mathrm{ses}=$

\begin{tabular}{|c|c|c|c|c|c|}
\hline \multirow[b]{2}{*}{ Capm } & \multicolumn{5}{|c|}{$\mathrm{Bpm}$} \\
\hline & 0,0605 & $0,1,0$ & 0,3 & $0: 5$ & Mitia Co \\
\hline \multicolumn{6}{|c|}{ (n) } \\
\hline 150 & $87: 10$ & 24,36 & 28,35 & 80,75 & $23: 640$ \\
\hline 100 & 25,8 & 26,61 & $28: 10$ & 2136 & $24,3 e$ \\
\hline eno & 46,12 & $4 \%, 199$ & $50: 43$ & $49: 31$ & $4 B: 34 A$ \\
\hline 400 & 26,30 & $36,8 \mathrm{z}$ & 48,83 & 50,42 & $40,34 \mathrm{~B}$ \\
\hline Media & $30,94 a$ & $34,27 a$ & $30.93 a$ & 35,34 & \\
\hline
\end{tabular}

$$
\text { Medja } 34,16 \text { dms }
$$

* Hedias seguidas de letras majusculas ne vertical e minusculas na horizontal iguais nầ diferem entre si pelo teste de Tukey ao nivel de 5 de probabilidade.

\subsection{Caules}

\subsubsection{Peso de matéria seca e diâmetro dos caules.}

De resultados de peso de materia seca dos

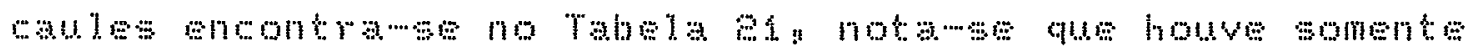
Weito do oa de maneira que a modia que se elevou o ca na solucto nutritiva aumentou o pos de materia seca do wale. Tgual resultado foj encontrado por burstrol (1964)

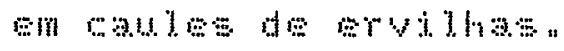

Com rejacto ao difnetro médio dos males a mesmo mumentoun con o aumento da dose de can conforne mostra o Tabela es. Tal resultadon concorda com os obtidos 


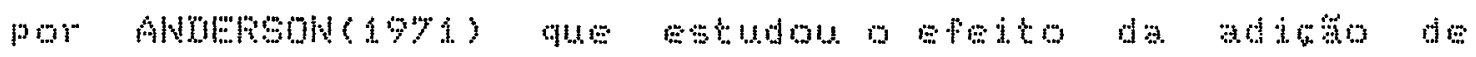
micronutrientes calcario gesso en mudas com un ano de idade: em condibses de wampo encontrando acreseimo no

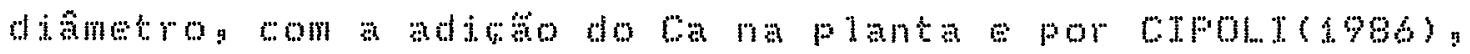

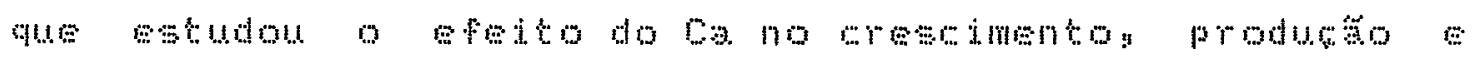

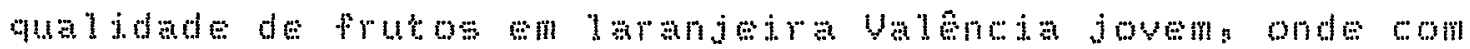

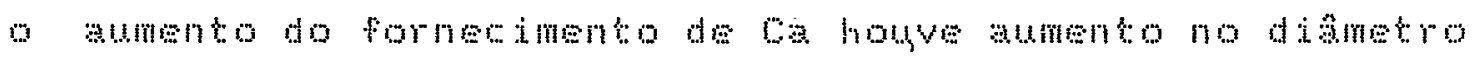

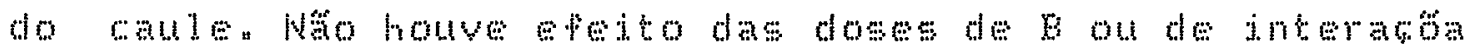

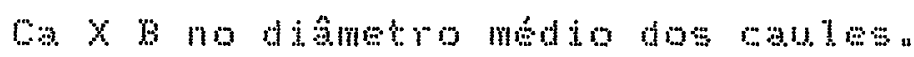

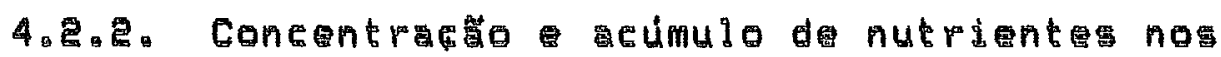 caules}

na mesma manedra que para as ratzes para os coules: os resultados de concentratio de $N$ o Fy apresentados nas Tabelas es o 25 respetivmente notames

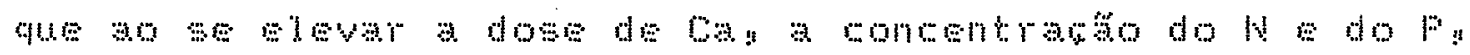
diminuiu sendo este fato decormente do mumento do poso do

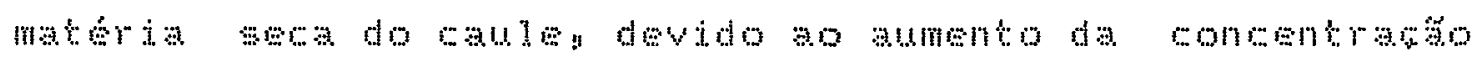
de ba ocorrendo assim o efeito de diluicho Fios

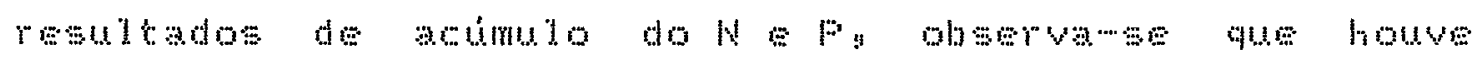
epedto somente da dose de ca como säo apresentados nas

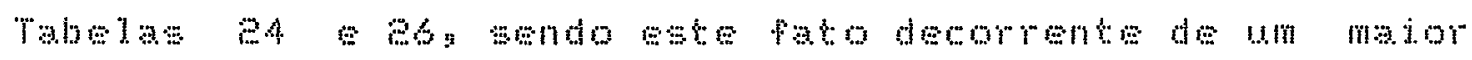

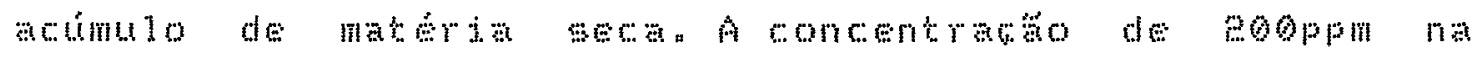
olutso foi a que conduriu aos matos actmulos de $N$ o nos calles "Tal efesto foj comprovado por fosson et all $(19 \% 9)$ "

Fara o s. os resultados de concentracto

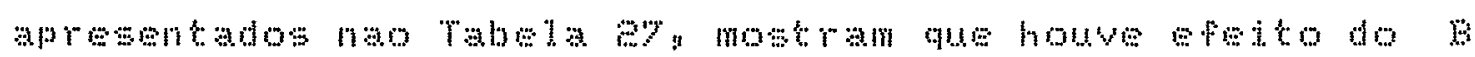




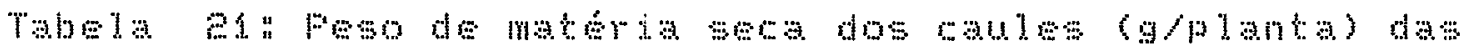
plantas de limosiro cravo (media de 4 repetioges?.

\begin{tabular}{|c|c|c|c|c|c|}
\hline \multirow[b]{2}{*}{ Gapm } & \multicolumn{5}{|c|}{$\mathrm{B}$ min } \\
\hline & $a, 0683$ & 9,125 & 0,80 & 0,5 & Motia \\
\hline 30 & $3: 39$ & 3 y 25 & 3,33 & 294 & 3,230 \\
\hline 100 & $3: 60$ & $4: 40$ & 3,29 & $3: 67$ & 3,740 \\
\hline 800 & $9: 99$ & 8,71 & 7,67 & 9,17 & $8,94 m$ \\
\hline 400 & 5,43 & $8,6 \%$ & 7,80 & 8.86 & $7: 53 \mathrm{E}$ \\
\hline Media $B$ & $1 ; 3,60 \%$ & 6,103 & 3,87 & 6,16 & \\
\hline
\end{tabular}

* Hedias seguidas de letras mainsculas na vertical e minusculas na horizontal iguais năo diferem entre si pelo teste de Tukey ao nivel de 5 de probabilidade:

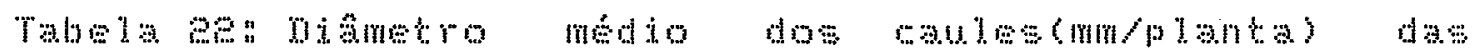
platra de limoeiro cravo cateda de 4 repetscoss?

$\mathrm{B} p \mathrm{~m}$

\begin{tabular}{|c|c|c|c|c|c|}
\hline \% ppin & 0,0620 & 0,125 & 0,8 & $0,: 0$ & Media Ca \\
\hline 130 & 5,86 & $5 \cdot 66$ & 5.62 & $3: 67$ & 7,708 \\
\hline 100 & 3,8 & 6,19 & 5,30 & 5.78 & 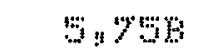 \\
\hline 200 & 8,07 & $8: 08$ & $8: 0 \%$ & 8.23 & $B_{711 \mathrm{~A}}$ \\
\hline 400 & $6: 9$ & $7: 34$ & $8: 33$ & $8,0: 5$ & $7: 7 \mathrm{~A}$ \\
\hline 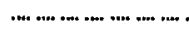 & . & . & 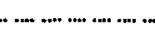 & (n) & 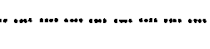 \\
\hline Media & $6: 674$ & $684 \pi$ & 6. & $6.99 a$ & \\
\hline
\end{tabular}

* Hédias seguidas de letras maísculas na vertical e minúsculas na horizontal iguais nắ diferer entre si pelo teste de Tukey ao nivel de $5 \%$ de probabi]idade. 


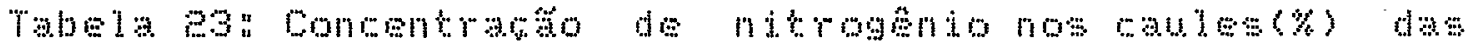
plantas de limosiro cravo cmédia de 4 repetistos;"

\begin{tabular}{|c|c|c|c|c|c|}
\hline \multirow[b]{2}{*}{ \% ppm } & \multicolumn{5}{|c|}{$\mathrm{gppm}$} \\
\hline & 0,0605 & 0,485 & 0,25 & 0.5 & Media $\mathrm{Ca}$ \\
\hline 50 & 1,60 & $2: 03$ & 1.82 & 192 & 1. $984 \mathrm{~A}$ \\
\hline 100 & $4,7 y$ & $1,8 \%$ & $1 \neq 1$ & 5.84 & $1.79 \mathrm{~A}$ \\
\hline 200 & 1. 44 & 1.61 & 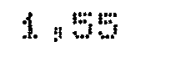 & $1 \div 4$ & 5.545 \\
\hline 400 & 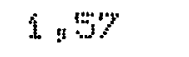 & 1,43 & is $: 390$ & 1,46 & 1.505 \\
\hline Modia & $1,59 \quad 3$ & $178=$ & $1,66 \div$ & 69 & \\
\hline
\end{tabular}

* Kédias seguidas de letras maiúculas na vertical a minúculas na horizontal iguais nä́ diferem entre si pelo teste de Tukey ao nivel de $5 \%$ de probabilidade.

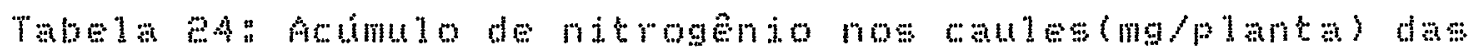
plantse de 1 inomo crivo cmodia de 4 repets $65=$

E p pin

\begin{tabular}{|c|c|c|c|c|c|}
\hline \multirow[b]{2}{*}{ Ga prom } & \multicolumn{5}{|c|}{ B pplin } \\
\hline & 0,068 & 0,183 & 0,20 & $0: 5$ & Media ca \\
\hline 50 & 54,63 & $65: 9$ & 40,84 & $36: 69$ & $89,60 \%$ \\
\hline 200 & $61: 53$ & $81: 40$ & $5: 5, y$ & 67,79 & 60460 \\
\hline 200 & $145: 63$ & 141,13 & $1 \in E, 11$ & 139,20 & $13 \mathrm{~g} \mathrm{se}$ \\
\hline 400 & $79: 13$ & $146: 8 \%$ & 120,58 & 128,30 & $112: 079$ \\
\hline Mats $\quad 8$ & $84: 7 a$ & 101 : 180 & $89: 75 \%$ & $70,00: 3$ & \\
\hline
\end{tabular}

* Hédis seguidas de letras maiusculas na vertical e minusculas na horizontal iguais năb diferen entre si pelo teste de Tukey an nivel de $5 \%$ de probabilidade. 


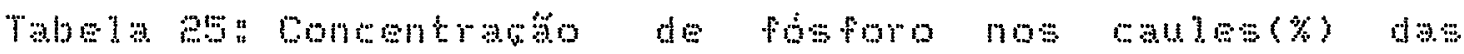
plantas de limosiro cravo lmedia de 4 $r e p+156 \%$ ?

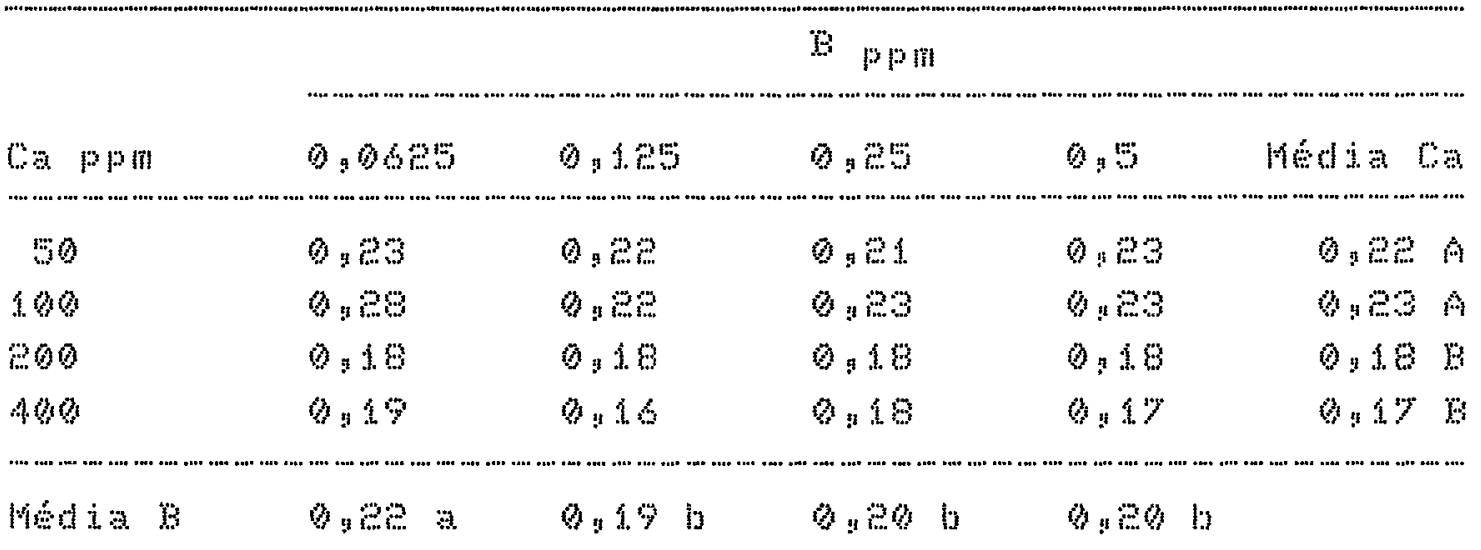

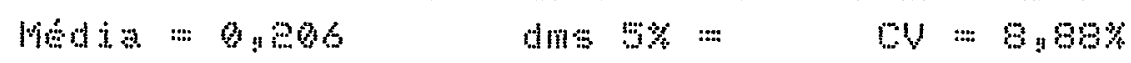

* Hedias seguidas de letras maísculas na vertical e minúsculas na horizontal iguais nắ diferen entre si pelo teste de Tukey ao nivel de $5 \%$ de probabilidade.

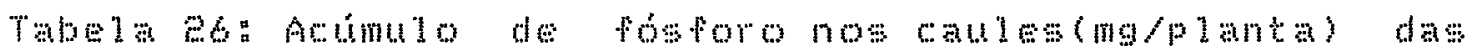
plantas de limoeiro cravo ciedia de 4 repetisis:

\begin{tabular}{|c|c|c|c|c|c|}
\hline \multirow[b]{2}{*}{$\mathrm{Capm}$} & \multicolumn{5}{|c|}{$\mathrm{E} \quad \mathrm{m} m$} \\
\hline & 0,0605 & 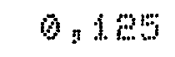 & 0,2 & 0,5 & Media fa \\
\hline 50 & 7,87 & 79 & $7: 19$ & $6: 69$ & 7 a 36 \\
\hline 160 & 10.68 & $9.7 \%$ & $7 y 50$ & $3: 38$ & $8: 920$ \\
\hline 200 & $18,7 \mathrm{i}$ & $1 \% 39$ & 14,65 & 17,09 & $36,46 \mathrm{~A}$ \\
\hline 400 & $9: 95$ & $13 \div 11$ & 13.93 & $14: 80$ & 1.8958 \\
\hline 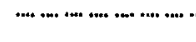 & . & 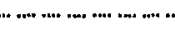 & . & . & 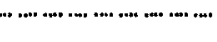 \\
\hline Hed $x$ & $12: 64: 3$ & $11,39 a$ & $10,82 \%$ & 16:74 & \\
\hline
\end{tabular}

: Hédias seguidas de letras maíisculas na vertical e minúsulas na horizontal iguaie ne diferen entre si pelo teste de Tukey a nivel de $5 \%$ de probabilidade. 


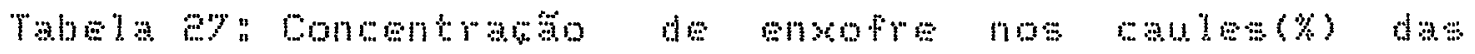
plantas de limosiro cravo lmedia de 4 repeticses?

\begin{tabular}{|c|c|c|c|c|c|}
\hline \multirow[b]{2}{*}{ Ca pFin } & \multicolumn{5}{|c|}{ Epm } \\
\hline & $0,060 \%$ & 0,125 & 0,20 & 0,13 & Média Ca \\
\hline 50 & 0,12 & 0,09 & $0 \div 0$ & $0: 10$ & $\theta, 11 A$ \\
\hline $\operatorname{son} \theta$ & $b_{3} 16$ & $a, 1.1$ & $0: 11$ & $a, 12$ & $0,12 A$ \\
\hline 200 & $0,1$. & 0,10 & $0_{9} 12$ & $0: 11$ & $0,11 \%$ \\
\hline 400 & 0,15 & $b_{13} 10$ & $a y$ & $9: 11$ & $\theta, 1 \mathrm{~A}$ \\
\hline Modia $\mathrm{B}$ & $0,14:$ & $a: 10 \mathrm{~b}$ & $a: 11 \quad b$ & a:A & \\
\hline
\end{tabular}

* Medias seguidas de letras maísculas na vertical e minúcculas na horizontal iguais näo diferen entre si pelo taste de rukey ao nivel de $5 \%$ de probabilidade.

nos tecidos dos caules de tal modo aue as concentrabes matores que o,bses pam $\mathrm{B}$ apresentaram toores no toodo semellantes setatisticanente entre s. menores que os

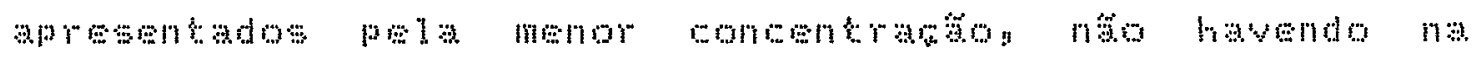
literatura trabnino que explique esse comportamento Nao

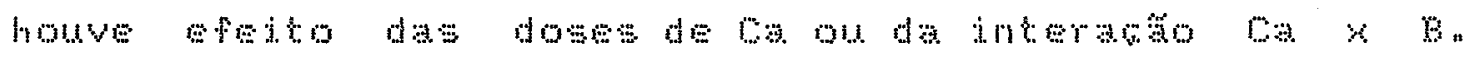

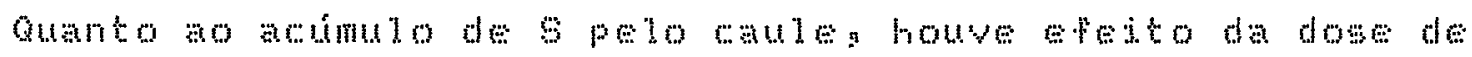

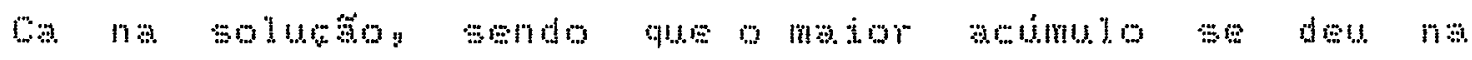
concentrato do eoppom de can como no caso de $\mathrm{N}$ e $\mathrm{F}^{*}$

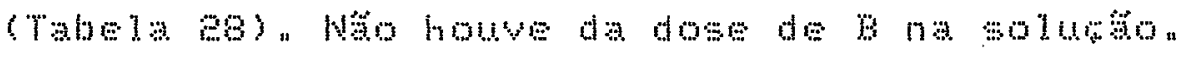

Os resultados de $K$ mostian que houve efejto

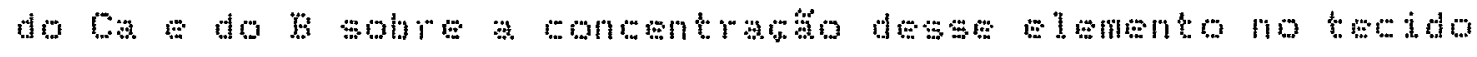

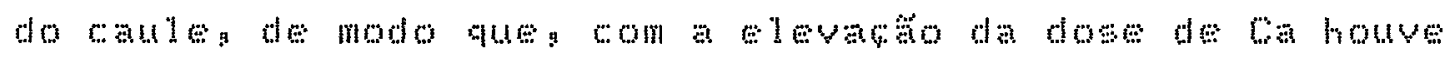




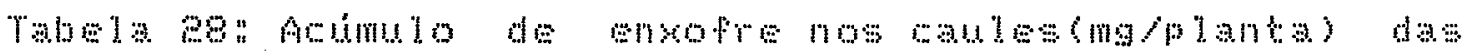
plantas de limouro cravo cmédia de repstis $6=3$

\begin{tabular}{|c|c|c|c|c|c|}
\hline \multirow[b]{2}{*}{ cap pain } & \multicolumn{5}{|c|}{$\tilde{A}_{p \mathrm{~m}}$} \\
\hline & 0.068 & 0,125 & 6,85 & $\theta,: 5$ & Hodin Ca \\
\hline 50 & 4.23 & 2.99 & 3,58 & 3,04 & 3,460 \\
\hline 100 & $S_{1} y_{1}$ & $4: 7 \%$ & 345 & $4,6 \%$ & $4: 660$ \\
\hline 200 & Ie: 1 & 9.40 & $9,9 e$ & 10.12 & $10.3 \mathrm{~A}$ \\
\hline 400 & 7.36 & 7,80 & $8,7 \%$ & $9: 74$ & $8: 40 \mathrm{~B}$ \\
\hline \multicolumn{6}{|c|}{$\begin{array}{llll}4 & \ldots\end{array}$} \\
\hline Métia $B$ & $7: 43 a$ & 6 4 & $6 ! 28$ & 6480 & \\
\hline
\end{tabular}

* Hédias seguidas de letras maiúculas na vertical e minúculas na horizontal iguais nä diferen entre si pelo teste de Tukey ao nivel de $5 \%$ de probabilidade.

Tabela po conentraso de potasio nos caules(\%) das plantes de limoiro cravo media de 4 repetioges?

\begin{tabular}{|c|c|c|c|c|c|}
\hline \multirow[b]{2}{*}{ mpin } & \multicolumn{5}{|c|}{$\mathrm{B} p p \mathrm{~m}$} \\
\hline & 0,068 & $0,12 \%$ & $0,8 \%$ & $a ;: ;$ & Media Ca \\
\hline 150 & 0.9 & 0,31 & $6: 83$ & 0.92 & $0.87 \quad 8$ \\
\hline 190 & $9: 93$ & $9: 82$ & $a_{1:} 9$ & $0,8 \mathrm{e}$ & $9,9 \%$ \\
\hline 200 & $1 ; 1$ & 0.99 & 1000 & $1: 03$ & $1.03 \mathrm{~A}$ \\
\hline 400 & $1: 0 \%$ & 9.96 & 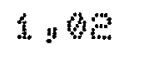 & 0.97 & $190 \mathrm{~A}$ \\
\hline Mads $\mathrm{B}$ & $1.0 \mathrm{a} \quad \mathrm{a}$ & 9,9010 & $0: 98 \mathrm{~b}$ & 0,93 & \\
\hline
\end{tabular}

* Kodias seguidas de letras maideculas na vetical e minusculas na horizontal iguais năo diferen entre si pelo teste de Tukey ao nivel de $5 \%$ de probabilidads. 


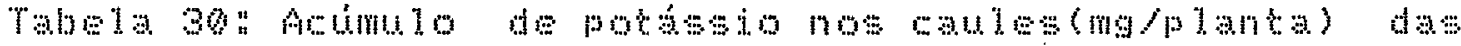
plantas de limosiro cravo limedia de 4 repetises;

\begin{tabular}{|c|c|c|c|c|c|}
\hline \multirow[b]{2}{*}{ Ca ppm } & \multicolumn{5}{|c|}{$E p p m$} \\
\hline & 0.6625 & $\theta_{n} 180$ & $0,8 \%$ & 0,9 & $11 \mathrm{Ca}$ \\
\hline 150 & 31,4 & 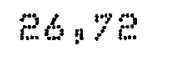 & 279 & $277_{a} 01$ & $28,27 \%$ \\
\hline 190 & 34,8 & 36,36 & $27: 34$ & $30: 40$ & $3 e: 10 \mathrm{~s}$ \\
\hline 800 & 110,17 & $86 \cdot 66$ & 78,45 & $94: 81$ & $9 \geqslant: \because 8 \mathrm{~A}$ \\
\hline 400 & 59,76 & 79.74 & $79: 90$ & $86: 19$ & 76,404 \\
\hline Modia & 50,913 & 57 a 37 & 53,40 & 57.69 & \\
\hline
\end{tabular}

口 Medias seguidas de letras maibculas na vertical e minusculas na horizontal iguais nea diferen entre si pelo teste de Tukey an nivel de $5 \%$ de probabilidade.

aumento da concentragio de $K:$ que para o 1 as

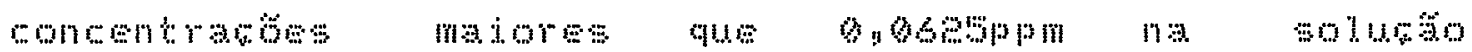
mpresentaram teones no towdo semelhantes estatistionente entre st menores me os anesunados por esta

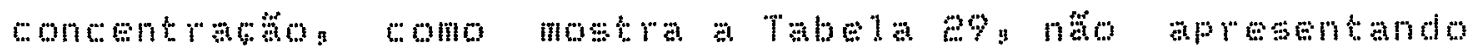

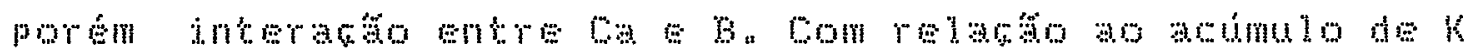
no trodon houve eferto somento de ca como mostra a Tabla 30, onde com o mumento do na soluto houve

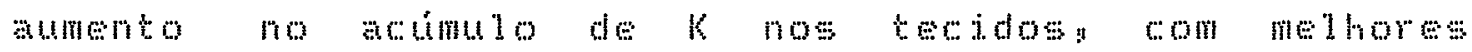

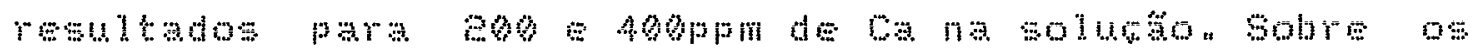
resultados encontrados na revisan existem trabalhos afirmando que com o aumento da conontraso do os na

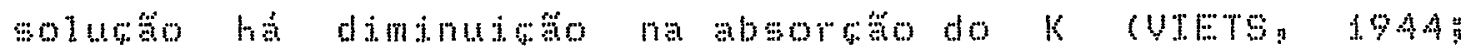




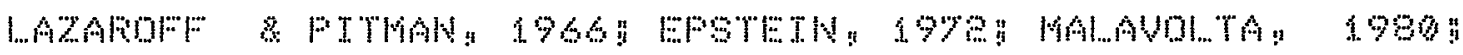

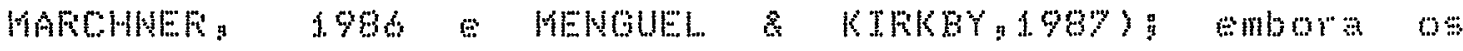

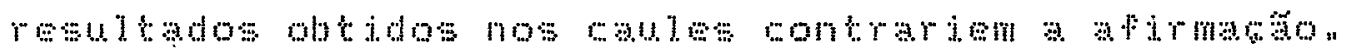

Fara o Mga os resultados de conomtata no herdo encontramse nao Tabela 31 observase que houve afeito somente do aumento do ba na solumio nutritiva

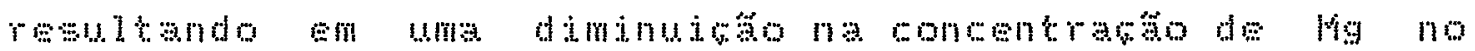
teodo do caule mostrando o weito de diluigio ja

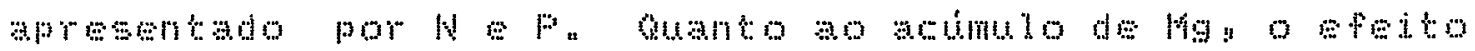
do Ca ocomen de maneira inversan ou sejan com o aumento

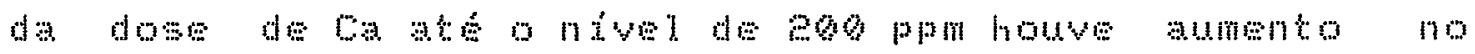
acumblo de Mg no tecido do cale haveno diminuigáno no actmulo dosto nutriente com o ammen do nivel do ro par

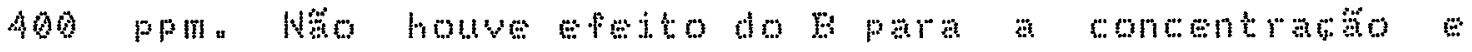
acumulo de Ma: como mostra rabela se. os resultados

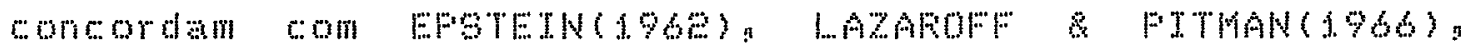

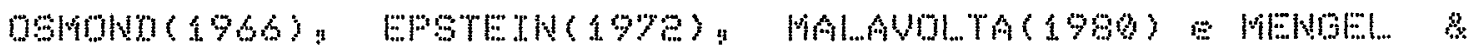
KTREY (1987),

Fara o cas os resultados de concentracto estao presentes na Tabela 33 y metrando haver efedro do

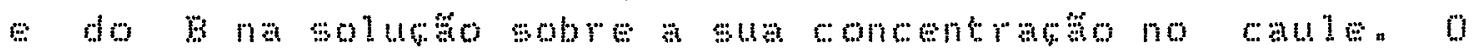
amento de dose de on na soluto nutritiva amentou o bear

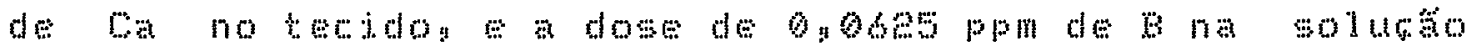

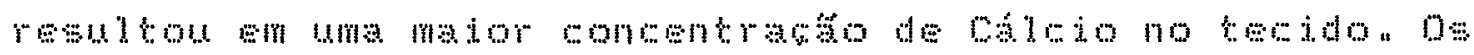
resultados de concentraço de Ca apresentam a segunto

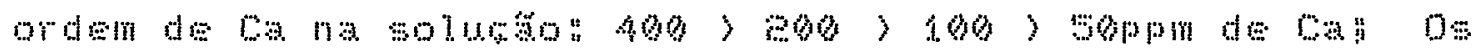
resultados de actmulo de ca apresentados na Tabola 34 


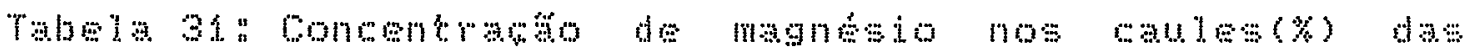
plantas de inomo travo lmedio de 4

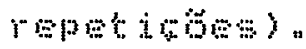

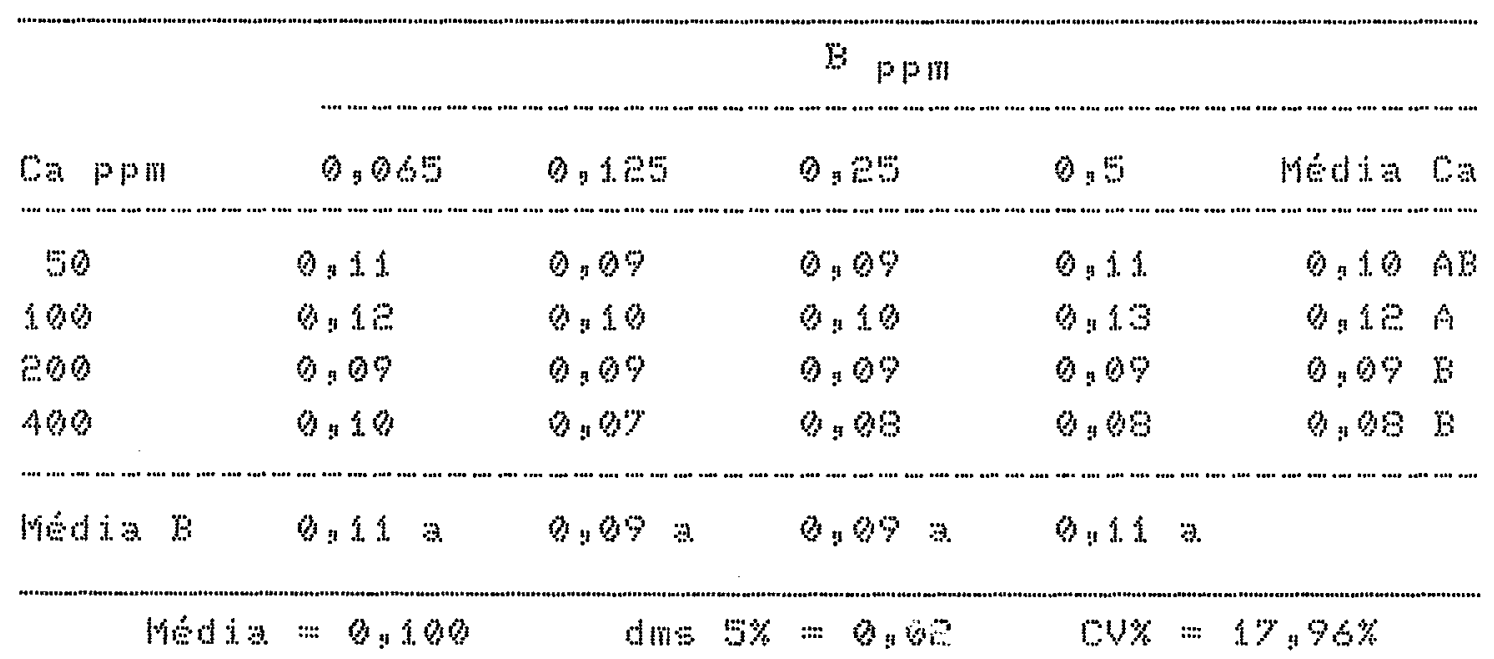

* Hedias seguidas de letras maísculas na vertical e ninusculas na horizontal iguais räo diferem entre gi pelo teste de Tukey ao nivel de $5 \%$ de probabilidade.

Tabela 3en Acumulo de manesio nos anulesolmglantas das plantos de limosio cravo cmedia de 4 repeticises.

\begin{tabular}{|c|c|c|c|c|c|}
\hline \multirow[b]{2}{*}{ cat ppn } & \multicolumn{5}{|c|}{ e ppm } \\
\hline & 0,060 & $0,18 \%$ & 6,25 & $0 \div$ & Medin Ca \\
\hline 50 & $3,9 e$ & 3,01 & 3,17 & $3,2 e$ & $3,31 \mathrm{C}$ \\
\hline 100 & 4,51 & $4: 67$ & 3,26 & 4,69 & 4,390 \\
\hline 200 & 9,09 & 8,37 & $7: 60$ & 0,38 & $0,36 \mathrm{~A}$ \\
\hline Ano & 4,72 & $6: 09$ & 6,76 & $y, 31$ & 6 y 26 \\
\hline
\end{tabular}

\begin{tabular}{|c|c|c|c|}
\hline Madia & 6 у ва & 5,53 & в в \\
\hline
\end{tabular}

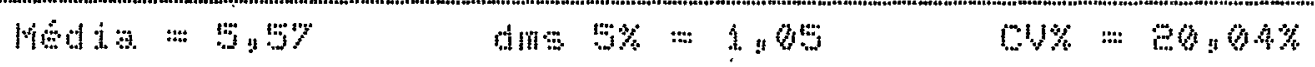

* Medias seguidas de letras maísculas na vertical e minúculas no horizontal iguais nău diferem entre si pelo teste de Tukey an nivel de $5 \%$ de probabilidade. 


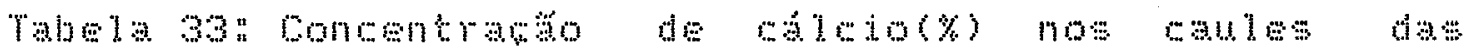
plantas de linotiro travo linedia de 4 repetisos?

\begin{tabular}{|c|c|c|c|c|c|}
\hline \multirow[b]{2}{*}{ Bapm } & \multicolumn{5}{|c|}{ E ppin } \\
\hline & 0.068 & $0,18 \%$ & 0.20 & $0: 1:$ & Media Ca \\
\hline 50 & 0,33 & $0,2 y$ & 0,80 & $0: 31$ & $0,30 \quad 4$ \\
\hline 100 & 0,48 & 9,36 & $0: 40$ & 0,47 & $0: 436$ \\
\hline 800 & 0.83 & 0,73 & 0.77 & 0,79 & $0,77 \mathrm{~s}$ \\
\hline 400 & $1: 02$ & 0,83 & $0, a$ & 0,76 & $0.801 \mathrm{~A}$ \\
\hline Msdia $B$ & $0: 66$ & 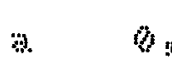 & $b$ & $\mathrm{ab}$ & $7 \mathrm{~b}$ \\
\hline
\end{tabular}

* Médias seguidas de letras maíiscuis na vertical e minusculas na horizontal iguais nato diferea entre si pelo teste de Tukey an nivel de $5 \%$ de probabilidade.

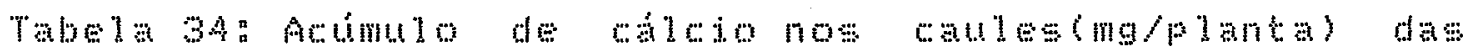
plantas de limosiro wrowe cmedia de 4 repeticosis

\begin{tabular}{|c|c|c|c|c|c|}
\hline \multirow[b]{2}{*}{ Ca ppm } & \multicolumn{5}{|c|}{ E $p \mu$} \\
\hline & 0,068 & 0,125 & $0,=3$ & 0,0 & Média ma \\
\hline 100 & 11,41 & $9, b 1$ & 9.6 & $9: 02$ & 9.908 \\
\hline 500 & $169 \%$ & 16403 & 13,23 & $1 \% 3 \%$ & 1 $1: 9 \%$ \\
\hline 800 & $82: 4$ & $62 \cdot 66$ & 80,80 & $68: 34$ & $68: 31 \mathrm{~A}$ \\
\hline 400 & 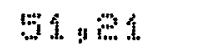 & $66 ! 40$ & 71,09 & $67: 4 \%$ & $64.03 \mathrm{~A}$ \\
\hline Mala & $46, A A=$ & $38,69 \quad$ & 39.67 & 40,54 & \\
\hline
\end{tabular}

* Médiss seguidas de letras maisculas na vertical e minusculas na horizontal iguais năb diferem entre si pelo teste de Tukey ao nivel de $5 \%$ de probabilidade. 
motram que houve eferto monente do aumento da dose do can

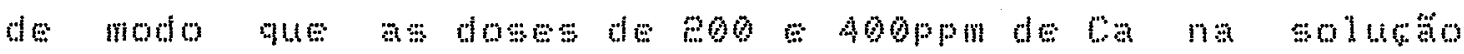

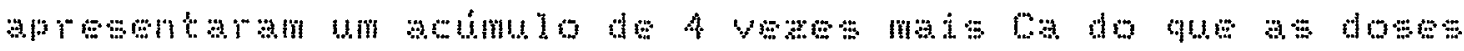

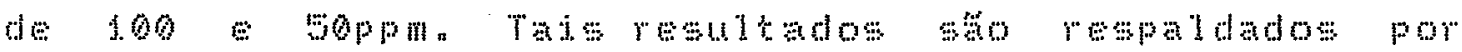

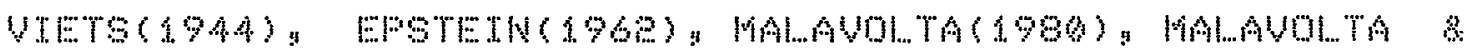
UTOLANTE: NETO(1999) MALAUOLTA E alia(1989).

A concentratoso de $B$ nos tecidos do cauls

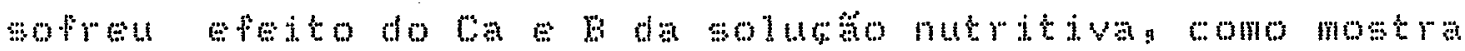

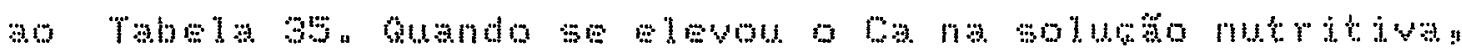
houve uma diminujato no teor do tecidon caracterizando o

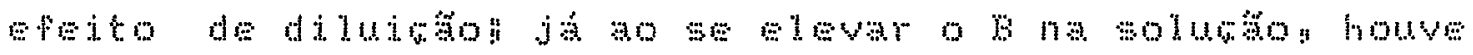

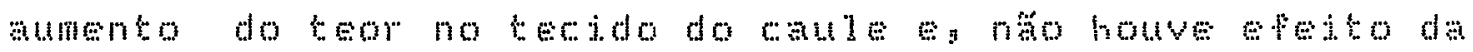

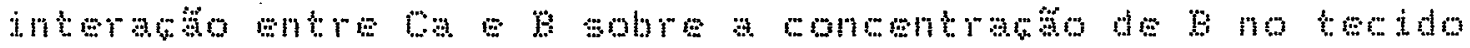

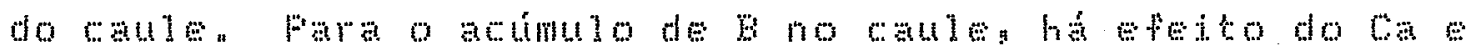

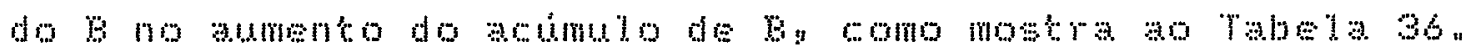
Tanto para o ca como para o 8 o aumento nas doses na

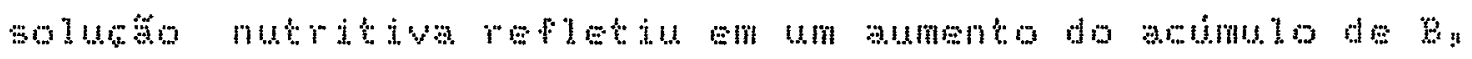

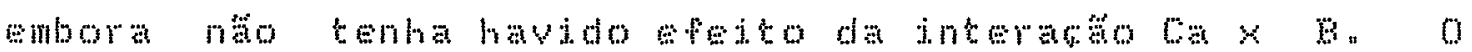

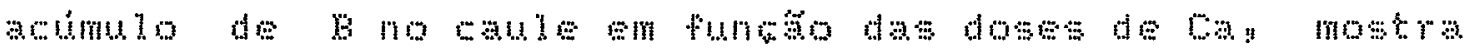
que os tratamentos con 200 e $400 \mathrm{ppm}$ de ca form corm de

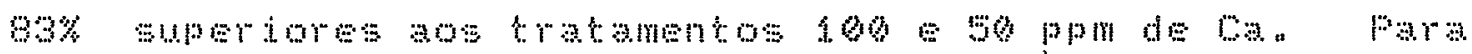
os tratamentos com 8 . o melhor resultado ocorreu para oy ppm de $\mathrm{E}$ na solug 


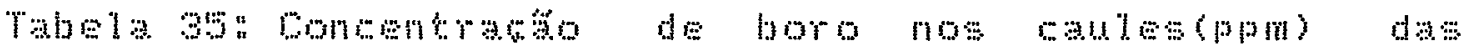
plantas de limoeiro oravo media de $A$ repets

\begin{tabular}{|c|c|c|c|c|c|}
\hline \multirow[b]{2}{*}{ Ca prin } & \multicolumn{5}{|c|}{$\mathrm{B} F \mathrm{Fm}$} \\
\hline & 0,068 & $0,1.84$ & 0,30 & 0,6 & Média Ca \\
\hline 130 & $24,2+3$ & $20,2 \ldots$ & 2150 & 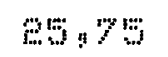 & $2 \mathrm{~B} \cdot 8 \mathrm{~A}$ \\
\hline 100 & en 13 & $19: 5 \%$ & $23: 00$ & $24 \%$ & $28: 62 A$ \\
\hline 000 & 15,75 & 16,23 & $18: 30$ & 17,00 & 46,878 \\
\hline 400 & $49: 7 \%$ & $16,7 \%$ & 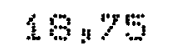 & 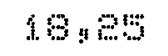 & $18: 37$ \\
\hline \multicolumn{6}{|c|}{ 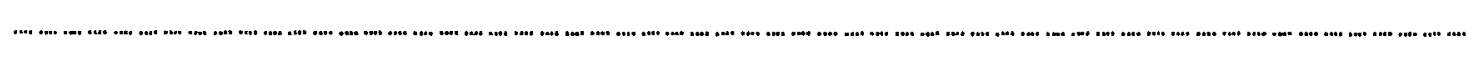 } \\
\hline Modia $\quad 8$ & $19,100 \mathrm{ab}$ & 10 y $18 \mathrm{~h}$ & $80,93 a$ & $21,4 \%$ & \\
\hline
\end{tabular}

* Hedias seguidas de letras maisculas na vertical e minúsculas na horizontal iguais nä́o difaren entre si pelo teste de Tukey an nivel de $5 \%$ de probabilidade.

Thbela 36: Actmulo de boro nos cales (ugplanta) das plantas de limosto rravo cmedia de repeticoses?.

\begin{tabular}{|c|c|c|c|c|c|}
\hline \multirow[b]{2}{*}{ sepm } & \multicolumn{5}{|c|}{ E ppn } \\
\hline & 0.6628 & $\theta, 12 \%$ & 0,25 & 0,5 & Media Ca \\
\hline 50 & 7.96 & 65,74 & 7.06 & $7 \mathrm{~g}, 97$ & $71.08 \mathrm{~B}$ \\
\hline 100 & $77,2$. & $8: 40$ & 80,27 & 70,30 & $63,74 \%$ \\
\hline 200 & 46538 & $140: 51$ & 446,81 & $106: 04$ & 1. $49: 4 \mathrm{AA}$ \\
\hline 406 & 95,06 & 198,89 & 146,46 & $16 \theta_{n} \%$ & $134,03 \mathrm{~A}$ \\
\hline \multicolumn{6}{|c|}{ 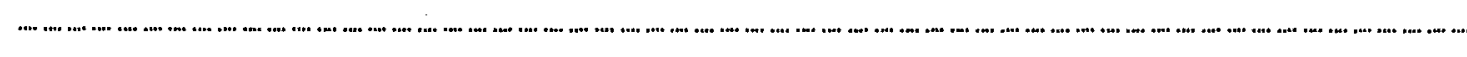 } \\
\hline Mitua & $99: 87 \mathrm{~h}$ & $106 \div 63$ & 1.1 .10 & $181: 1:$ & \\
\hline
\end{tabular}

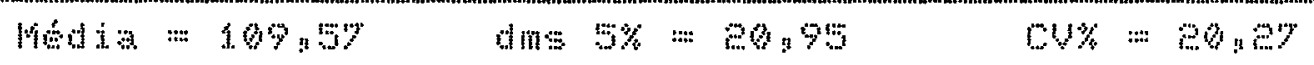

* Hédias seguidas de letras miusculas na vertical e minusculas na horizontal iquais năto diferem entre si pelo teste de Tukey ao nivel de $5 \%$ de probabilidade. 


\subsection{Folhas}

\subsubsection{Peso de matéria secag área foliar e número de folthas.}

Felos resultados de peso de materia secan area foldar numero de folhas notsos que houve efeito da dose de fas como mostram as Tabuas 37,38 e 39

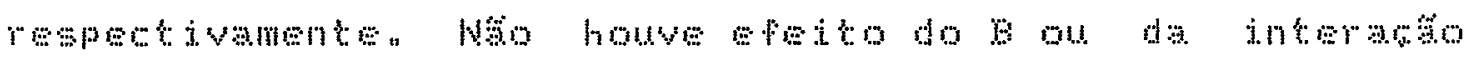
waxb. Tase resultados de numero de follas conomdam com

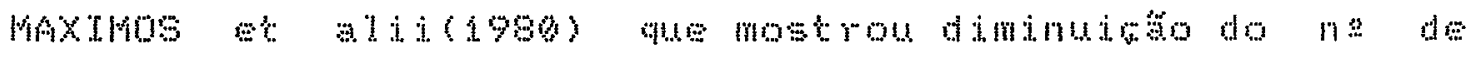

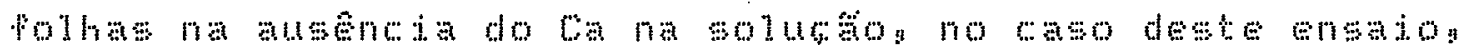
a prosenra do ca amontou nas 50 o na de polhas mas

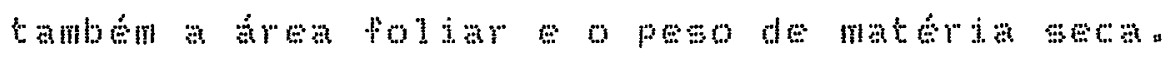

\subsubsection{Concentracão e acúmulo de nutrientes pelas folhas.}

Para os resultados de concentrato de N nas

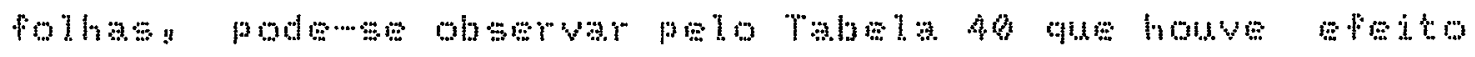
do aunento do ca na solutro Nutritiva diminuindo a conontrata do w na follas com o aumenco do or na soluctón oumto ao acumblo de Ng en fung da dose de ca

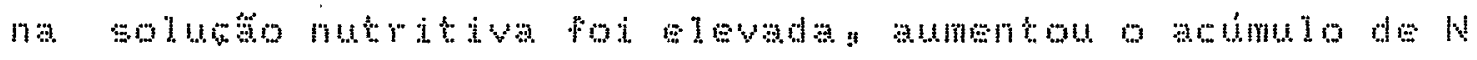
no tecido munentou ate a dose de eoo ppm cono mostra a 
Tabela $3 \%$ preso do matria seca das follasecalanta das plantas de limotio crivo bedia de repetioses.

\begin{tabular}{|c|c|c|c|c|c|}
\hline \multirow[b]{2}{*}{ Ca ppli } & \multicolumn{5}{|c|}{$\mathrm{s} p \mathrm{pm}$} \\
\hline & 0,068 & 0,123 & 0,80 & 0,9 & Menta ca \\
\hline 50 & 2.66 & 2,88 & 3,00 & 2,85 & $8: 8 \%$ \\
\hline 100 & 3,64 & 4.10 & 3,86 & 3,48 & $3: 48$ \\
\hline 800 & $8: 7$ & $7: 56$ & 7,15 & 0,14 & $7.89 A$ \\
\hline 400 & 4,74 & $7: 48$ & $7_{5} 74$ & $3_{43}$ & $Y: 94 \mathrm{~A}$ \\
\hline Modia $\mathrm{B}$ & $4: 97 \%$ & $3 ! 43$ & $3: 443$ & $5: 68$ & \\
\hline
\end{tabular}

* hedias seguidas de letras maisculas na vertical a anúsculas na horizontal iguais hä̆ diferen entre si pelo teste de Tukey ao nivel de sh de probabilidade.

Tabela 38" hrea foliar (cme/planta) das plantas da 1 imosiro oravo (média de A repeticose)"

\begin{tabular}{|c|c|c|c|c|c|}
\hline \multirow[b]{2}{*}{ Ca $p p m$} & \multicolumn{5}{|c|}{$8 \quad \mathrm{pm}$} \\
\hline & 0.0625 & 0,183 & 0,85 & 0.5 & Méda Ca \\
\hline 50 & 300,99 & $32 \%, 89$ & 398,55 & 301,70 & $337,78 \mathrm{~B}$ \\
\hline $10 \%$ & $404: 66$ & 448,95 & 430,78 & 37340 & $43,41 \mathrm{E}$ \\
\hline 200 & 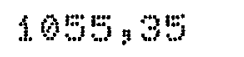 & 897,17 & 77790 & $976: 96$ & 96397 \\
\hline 400 & $88 e: 96$ & 813,73 & 931.90 & $884: 23$ & $803.05 \mathrm{~A}$ \\
\hline Mod: 8 & 585.973 & $616: 43$ & $634,48 \%$ & $639,3 i$ & \\
\hline
\end{tabular}

* Hédas seguidas de letras maísculas na vertical e minusculas na horizontal iguais nẩ ditrerem entre si pelo teste de Tukes ao nivel de $5 \%$ de probabilidade. 
Tabela 39 " Wimero de follas die plantos de linowiro cravo (media de 4 repetsoges).

\begin{tabular}{|c|c|c|c|c|c|}
\hline \multirow[b]{2}{*}{ Capm } & \multicolumn{5}{|c|}{$8 \mathrm{ppm}$} \\
\hline & $0,6,68$ & 6 y. & 0,25 & $0: 9$ & Media $\mathrm{Ca}$ \\
\hline 60 & 54,00 & $47: 00$ & ; & 50,26 & 51.128 \\
\hline 500 & $\% 9,00$ & 46,00 & 74,00 & $39 \%$ & $5 \%, 81 A B$ \\
\hline 800 & $9 \%, 78$ & $10 \%, 75$ & $99: 30$ & $98: 50$ & $509,37 \mathrm{~A}$ \\
\hline 400 & $74: 00$ & 68,75 & $134: 60$ & $73: 60$ & $67: 68 \mathrm{~A}$ \\
\hline Media $\mathrm{B}$ & $71: 68 \mathrm{a}$ & $60,00 \mathrm{a}$ & $90,31 \mathrm{a}$ & 67.00 & \\
\hline
\end{tabular}

* Médias seguidas de letras maiúsculas na vertical $e$ minusculas na horizontal iguais năo diferen entre si pelo teste de Tukey ao nivel de sto de probabilidade.

Tabela 4on Concentrano de nitrogento nas follase(\%) das platras de limosiro cravo bedia de repetis $60 ;$

\begin{tabular}{|c|c|c|c|c|c|}
\hline \multirow[b]{2}{*}{ OPpm } & \multicolumn{5}{|c|}{$\mathrm{B} p \mu n$} \\
\hline & 0.0685 & 0.185 & $0.8 \%$ & $0,: 3$ & Media Can \\
\hline 50 & 3.3 & $3: 31$ & 3.56 & 299 & $346 \mathrm{~A}$ \\
\hline 100 & 3,01 & 2.96 & 3,26 & $3: 34$ & 3 ? $4 \mathrm{AB}$ \\
\hline 200 & 2,76 & 2.88 & 2.66 & $2: 90$ & ? $80 \mathrm{~B}$ \\
\hline 400 & $8: 92$ & $2,8 \%$ & $3: 64$ & : : 58 & $2.93 \%$ \\
\hline Media $\mathrm{s}$ & : 98 & 2.99 & $3: 11 \mathrm{~m}$ & 2.963 & \\
\hline
\end{tabular}

* Hedias seguidas de letras maícculas na vertical e minúculas na horizontal iguais não diferem entre ai pelo teste de Tukgy ao nivel de sy de probabilidade. 


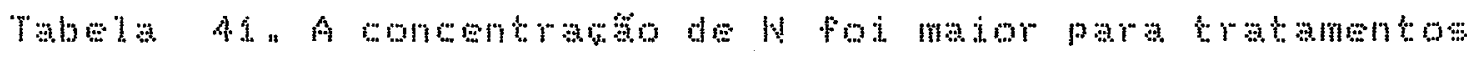

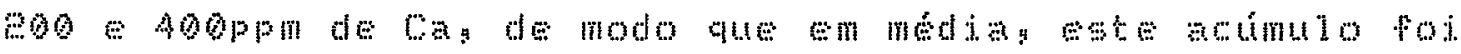

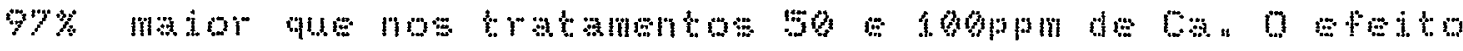

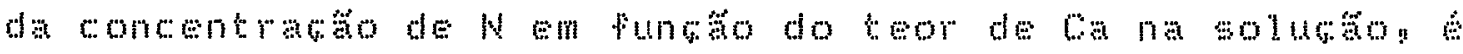

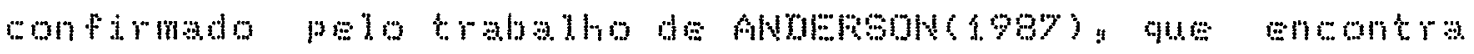

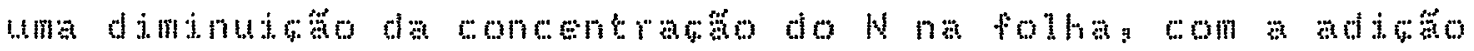

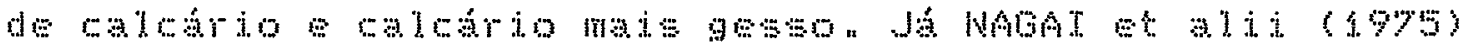
ostudando a jnterano entre os nutrientes arraves do

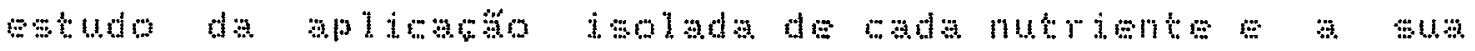
comrelacto com os demas nutrientes da polna afimaram

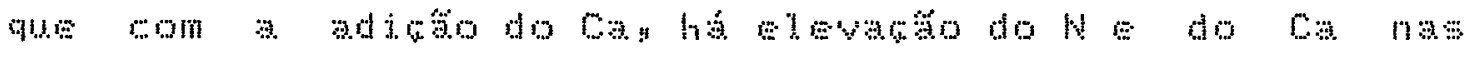
Pollows

Felos resultados de conentrato de pro na

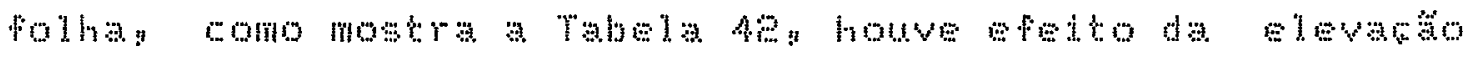

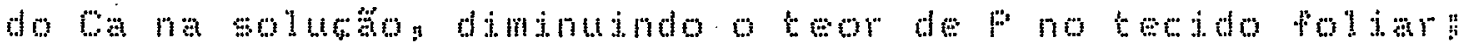

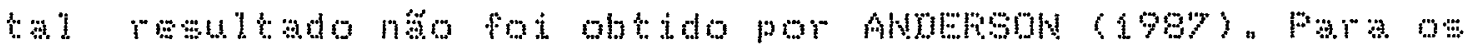

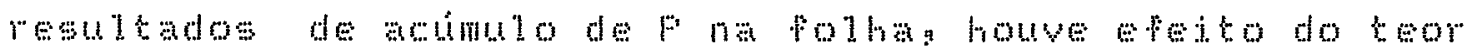

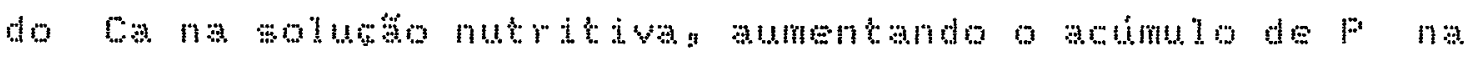

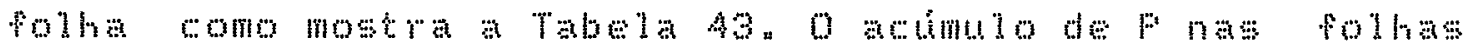

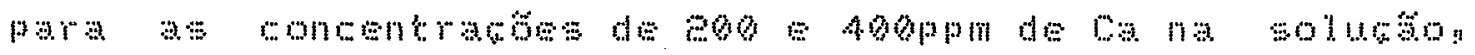
form erm de $30 \%$ mator que o promovido por 100 e woppm

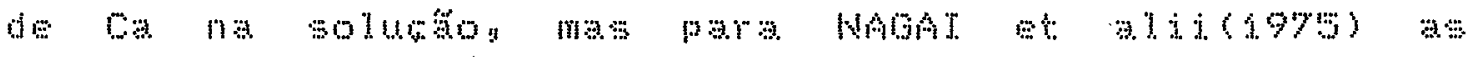
respostas da adifa de Ca sobre o teom de fina rolha náo

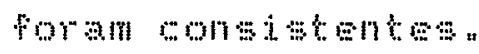

Os resultados de concentratio de $s$ na tolla estäo presentes na Tabela A4 onds mostra no ter havido eferto eignificativo de can $B$ da a interaso oa $x$ 
Tabela an Acumblo de nitrogindo nas follase(molalanta) da plantas de limoeiro cravo cincolia de 4 repetsoses

I $p: m$

\begin{tabular}{|c|c|c|c|c|c|}
\hline Ca ppln & 0,060 & $0,1,2=$ & 0,20 & 0.6 & Media Ca \\
\hline 100 & 86,01 & 95,78 & 104,39 & 85,16 & $9 e: 8 e \mathrm{~B}$ \\
\hline 100 & 100,13 & $1 \% 0,7$ & $123: 1$ & $115: 98$ & $11 \%$ : $07 \mathrm{Q}$ \\
\hline 200 & 236,2 & 216,5 & 198,33 & 233,49 & $219.63 \mathrm{~A}$ \\
\hline 400 & 131,18 & 603,48 & $23: 48$ & 21641 & $19646 \mathrm{~A}$ \\
\hline
\end{tabular}

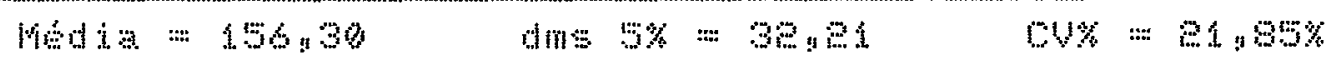

* Hédias seguidas de letras maísculas na vertical e minúculas na horizontal iguais näo diferem entre si pelo teste de Tukey an nivel de $5 \%$ de probabilidade.

Tabela Aen conentraso de postoro nas pollas $(x)$ das

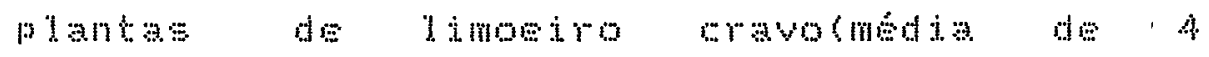
repeticeses).

\begin{tabular}{|c|c|c|c|c|c|}
\hline \multirow[b]{2}{*}{ mapil } & \multicolumn{5}{|c|}{$\mathrm{B} p \mathrm{pm}$} \\
\hline & 0,0605 & 0,183 & 0,20 & 0,15 & Media Ca \\
\hline 150 & 0.26 & 0,26 & 0,26 & 0,21 & $0.80 \mathrm{~A}$ \\
\hline 100 & 0,3 & by & $0,2 e$ & 0,20 & $a_{1}: 28$ \\
\hline 200 & 0,15 & 0.16 & 0,10 & $0,1.7$ & $\theta_{\mathrm{g}} \mathrm{t} 6 \mathrm{AB}$ \\
\hline 490 & 0,17 & $0,1.6$ & 0,17 & $\theta: 15$ & $0: 166 \mathrm{~B}$ \\
\hline 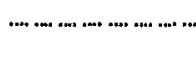 & . & 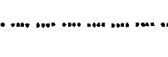 & 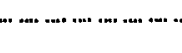 & (1..................... & 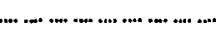 \\
\hline Mosis & $0,21 \quad 0$ & 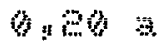 & $\theta, 80$ & 0.19 & \\
\hline
\end{tabular}

* Hédias seguidas de letras maibculas na vertical e minusculas na horizontal iguais näo diferel entre 5 pelo teste de Tukes a nivel de $5 \%$ de probabilidade. 
Basendo tais resultados rambin form obtas por crioln (196) ANolensow (1987) nos tratamentos onde utilizaram

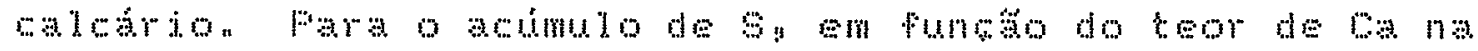

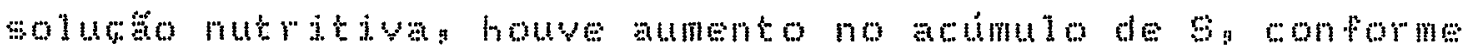
mostra a Tabela 45 tendo se dostacado os tratamentos eoo

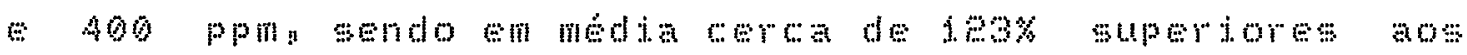

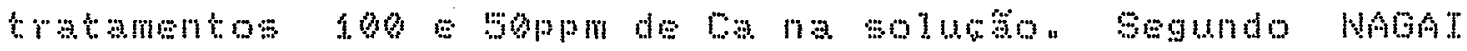
et ali (1975) as resposas da adiso de ca sobre a concentranto do 5 na follan tamben nas foram consistontas Fara conontratio de $k$ na follag cono mostra a Tabola 4 a am functo do toor de oa na soluse nutritiva houve elevaga no teor de k na follag enquanto 4ue a lovato da dose de ba diminuiu oteor de K na

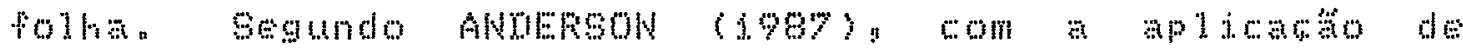

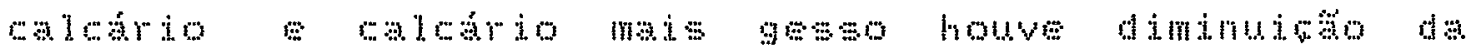

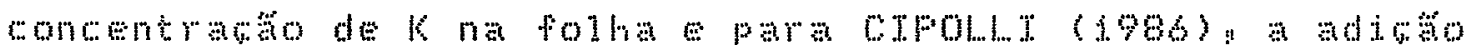

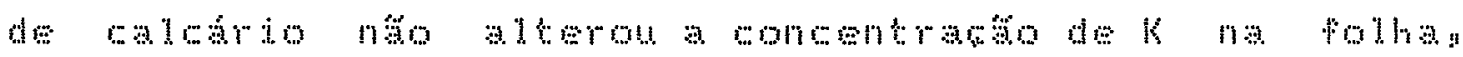
entretanto aplicando gesson houve diminuigon com a

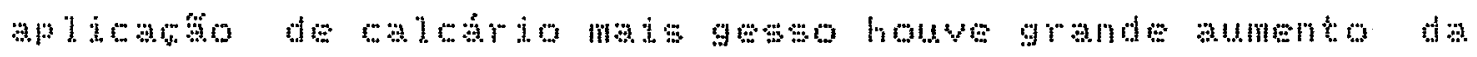

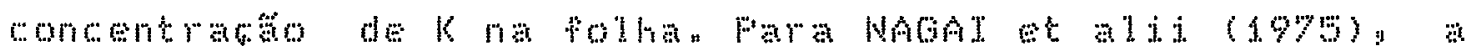

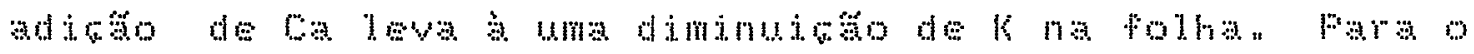
actimulo do $K$ na folma somente houve eferto da dose do oan como mostra a Tabla $4 y_{\text {a }} 0$ mator actmulo de ca ocorreu no tratamento eooppm de ca na solucăo nutritiva sendo

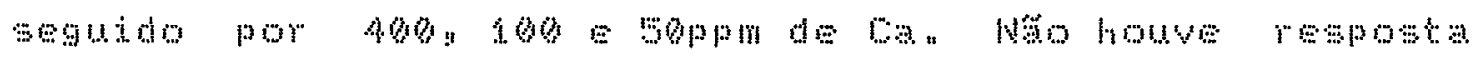

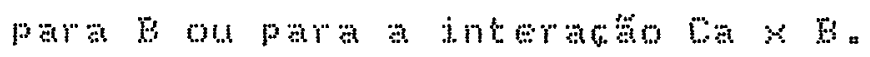


Fra os resultados de conoentrato de can apresentados na Tabela 48, verificouse que houve efeito

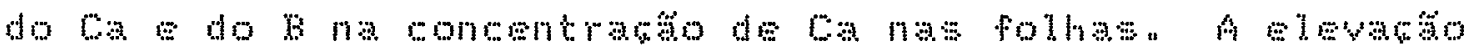
do Ca na solurăo resultou em um aumento ma concentrata

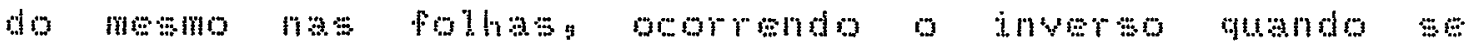

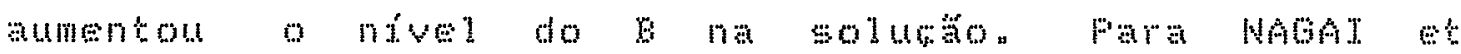

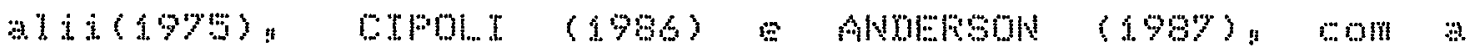

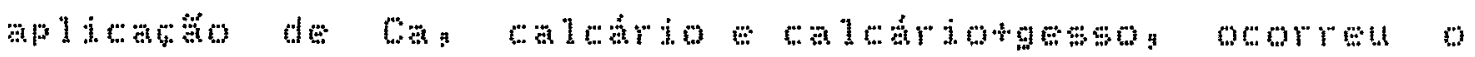
aumento do teor de oa nas follas nto havendo intomacos sobre a mo do 5 com relatio ao actmo de ca pelas

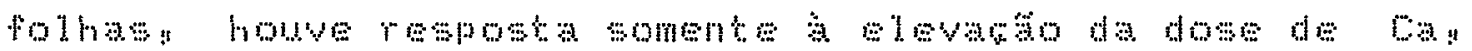
que promoveu mumen no acumalo de ca como mosta a Tabla 49" o mator acumb se deu nos tratamentos tho 200ppm de ca na solucton que se apresentaram orma de 7 y vese mator do que o ammlo promovido pelos

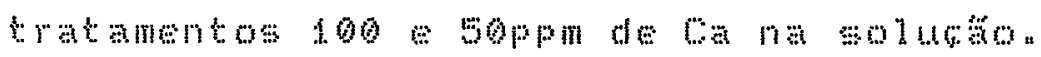

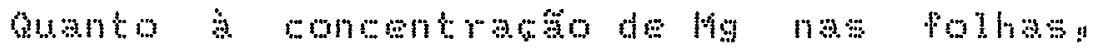
esta fod influenciada pelo aumbo dos niveds de can womo

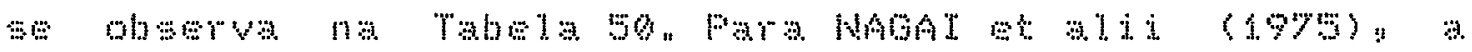
adifo de Ca leva a uma diminution do teor de Mg nas

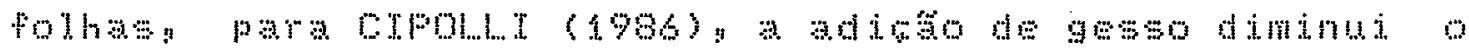

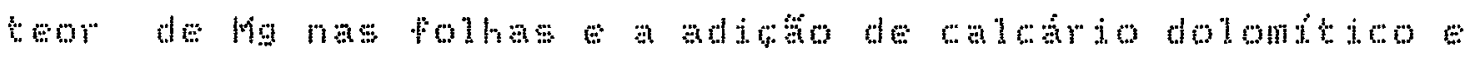

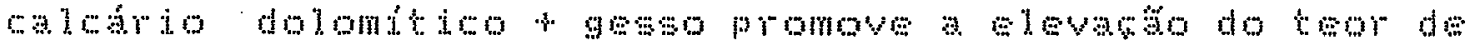

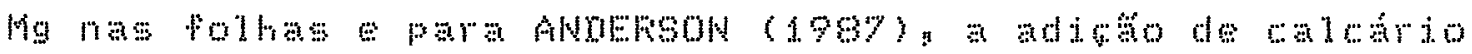

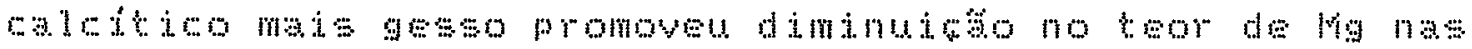
polbas e a adisto de calcaro dolomitio elevou o teor de Ha nas follas a cunto aos resultados de actimulo do Ma nas

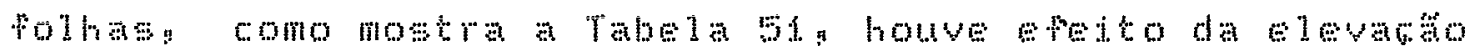

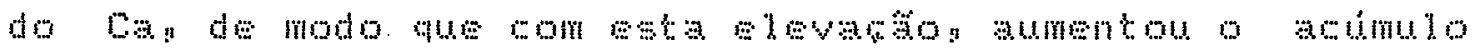
de lig na folna os tratamentos eoo w $400 \mathrm{pm}$ de ca na

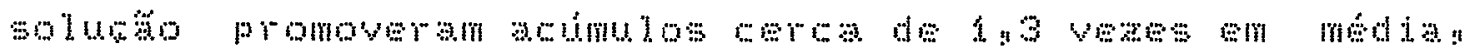


superiores abs cratamentos 100 soppon de ba

Fare a concentrano de 8 nas follas: houve efoto do or do s. como mostra a Tabla be do tal modo que a elevaño da dose de ca na soluca resultou en decrescimo nos teores do 8 nas polnas a com a elevaro da dose de 8 na solucto houve mumento no teor de 8 nas

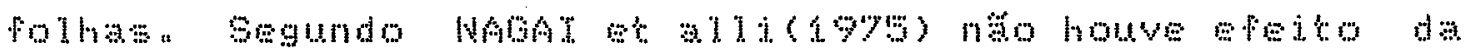
adjaxo de ca sobre o teor de nas folhas a ouanto ao

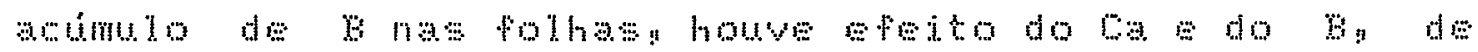
modo aue com a elevario das doses do ca e do 8 na

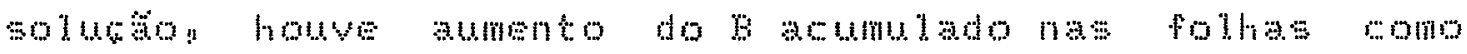

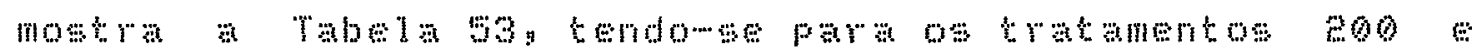

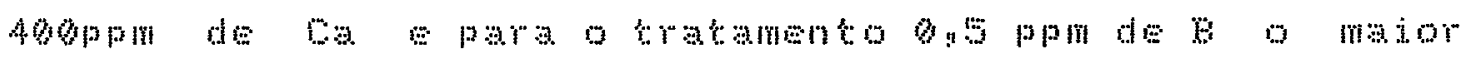
aimulo de $\mathrm{B}$ na folha

Tabela 45: Acumulo de enkolle nas follas (mo/planta das plantes de limotiro mavo cmedia de 4 repeticoses?

\begin{tabular}{|c|c|c|c|c|c|}
\hline \multirow[b]{2}{*}{$\mathrm{Capm}$} & \multicolumn{5}{|c|}{$\beta \quad p \rho m$} \\
\hline & 0,068 & $0,12 \%$ & $0, \ldots$ & $0,: 3$ & Media ca \\
\hline 50 & 8,3 & 9,39 & 9,06 & 7,36 & 6,580 \\
\hline 160 & $11: 07$ & 10,31 & $9: \because 4$ & 7,65 & $10 \div 140$ \\
\hline 300 & 24,96 & $28,7 y$ & 20,65 & 29,2 & $22.65 \mathrm{~A}$ \\
\hline mon & 1. 1. 97 & 20,80 & $8 \% 9$ & $80 ! 91$ & $19 \div 375$ \\
\hline Media $\theta$ & $14: 043$ & $15,83 a$ & 1 15998 & 19,88 & \\
\hline
\end{tabular}

"Hedias seguidas de letras maísculas na vertical e minusculas na horizontal iguais nắ diferen entre si pelo teste de Tukey an nivel de $5 \%$ de probabilidada. 


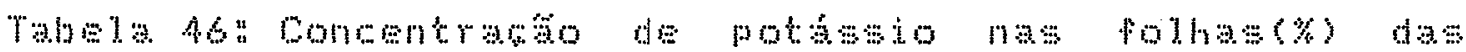
plantas de limosino cruvo cmedia die 4 $r e p e t i c 8 s ;$

\begin{tabular}{|c|c|c|c|c|c|}
\hline \multirow[b]{2}{*}{ Capm } & \multicolumn{5}{|c|}{$E \quad p p m$} \\
\hline & 0.068 & 0,123 & 0,25 & $6: 5$ & Media Ca \\
\hline 50 & $2: 70$ & $2: 5$ & 2.69 & $2: 34$ & $2,67 \mathrm{~B}$ \\
\hline 100 & 2.59 & e.s\% & 8,30 & $2: 47$ & $8: 43 B$ \\
\hline 200 & 3,03 & 3.08 & 2,80 & 2.98 & E:97A \\
\hline 400 & $3: 24$ & 206 & e 6 & $2: 47$ & 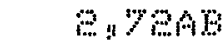 \\
\hline Modia $B$ & 2 , 89 & $e \div 6 m a b$ & $2: 68 \mathrm{ab}$ & $B: \div 40$ & \\
\hline
\end{tabular}

* Medias sogudas de letras maicculas na vertical a minisculas na horizontal iguais nän diferen entre si pelo teste de Tukey an nivel de st/ de probabilidade.

Tabela A7 Actimulo de potassio nas follaso(mg/planeal das planks de limotio mino cmedia de repetictoses?

\begin{tabular}{|c|c|c|c|c|c|}
\hline \multirow[b]{2}{*}{ \% $\mathrm{PPm}$} & \multicolumn{5}{|c|}{ 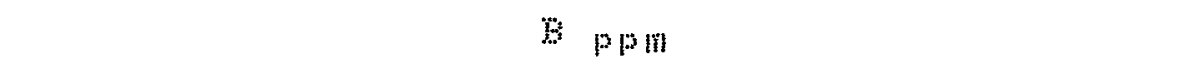 } \\
\hline & $0,06 e^{25}$ & $\theta, 1,3$ & 0,25 & 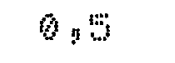 & Media Ca \\
\hline 60 & 72.31 & 79,83 & 80,11 & 67.33 & 73.40 \\
\hline 100 & 79.92 & $96.9 \%$ & $8 \%: 94$ & $84: 47$ & $90: 80 \%$ \\
\hline 800 & $8 \% 8,41$ & 235,01 & $267: 67$ & $23 x, 13$ & $233,0 \% \mathrm{~A}$ \\
\hline$A 00$ & 145,87 & $18 \%: 6$ & 20 :4\% & 20999 & $184: 498$ \\
\hline \multicolumn{6}{|c|}{ 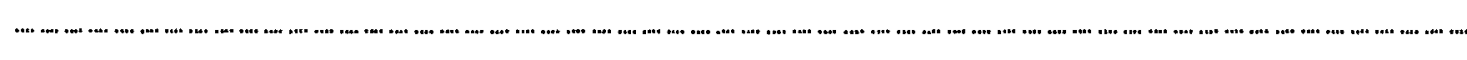 } \\
\hline Misio & $1.40,480$ & $1.47,69$ & $1.44,54$ & $147 \geqslant 3$ & \\
\hline
\end{tabular}

$$
\text { Media }=44,44 \quad \text { ans } 5 \%: 34,6 \% \text { ov }=23,2 \%
$$

* Hédias seguidas de letras maísculas na vetical e minúsculas na horizontal iguais năd diferer entre si pelo teste de Tukes ao nivel de $5 \%$ de probabilidade. 


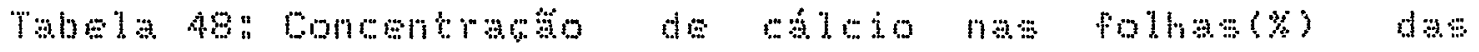
plantas de limojiro cravo bedia de 4 repetisos?

\begin{tabular}{|c|c|c|c|c|c|}
\hline \multirow[b]{2}{*}{ Ca ppin } & \multicolumn{5}{|c|}{$\mathrm{SPF}$} \\
\hline & 0,0605 & 0,18 & 0,25 & 0,5 & Media Ca \\
\hline 150 & 0,47 & 0,30 & 0,35 & 0,33 & 0,39 \\
\hline 100 & 0,50 & $3: 41$ & 0.46 & 0,4, & 0,460 \\
\hline 200 & 1.44 & 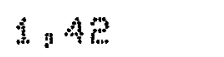 & 1.33 & $1: 36$ & 1,30 \\
\hline $40 \%$ & $1: 89$ & A. $: 60$ & 1.67 & 1. $: 3 \%$ & $1.63 \mathrm{~A}$ \\
\hline Mons B & $1,00 \quad$ & $0,96 \mathrm{ab}$ & $0,95 \mathrm{ab}$ & $0,8 \odot$ & \\
\hline Me & $=0,968$ & dms $5 \%$ & $:=0.46$ & w\%: & $7,76 \%$ \\
\hline
\end{tabular}

* Hédias seguidas de letras majuculas na vertical e minisculas na horizontal iguais nắ diferea entre si pelo teste de Tukey an nivel de $5 \%$ de probabilidade.

Tabela 49: Aclimulo de caloio nas folbas(mglelonta das plantos do limosiro crovo cmedia dis 4 repeticises?

$\mathrm{S} p p \mathrm{~m}$

\begin{tabular}{|c|c|c|c|c|c|}
\hline$\therefore \quad p p \mathrm{~m}$ & 0,0685 & 0,125 & 0,28 & 0,5 & Media ba \\
\hline 50 & 1292 & 10.99 & 10,80 & $9: 48$ & $10 ; 748$ \\
\hline 100 & $18: 06$ & 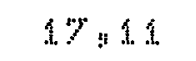 & $17: 49$ & $19: 90$ & $17: 4 \mathrm{~B}$ \\
\hline 800 & $1 \% 3,63$ & $106: 17$ & 95,68 & 509,16 & $108: 65 \mathrm{~A}$ \\
\hline 400 & 83,83 & $1 \mathrm{~A} 5,64$ & $\operatorname{sen} 37$ & $113: 47$ & A $1 \mathrm{~A}$, \\
\hline \multicolumn{6}{|c|}{.... } \\
\hline Mitia & $59: 84 a$ & $62,53 \mathrm{a}$ & $68: 93$ & $6 e_{1} 40$ & \\
\hline
\end{tabular}

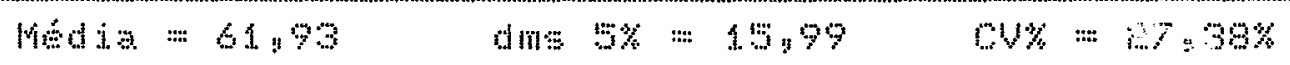

* Médias seguidas de letras maífculas na vertical e minisculas na horizontal iguais näo diferem entre si pelo teste de Tukey ao nivel de 57 de probabilidade. 


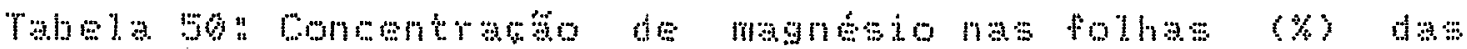
plantas de linoeino cravo (media de 4 repeticses;

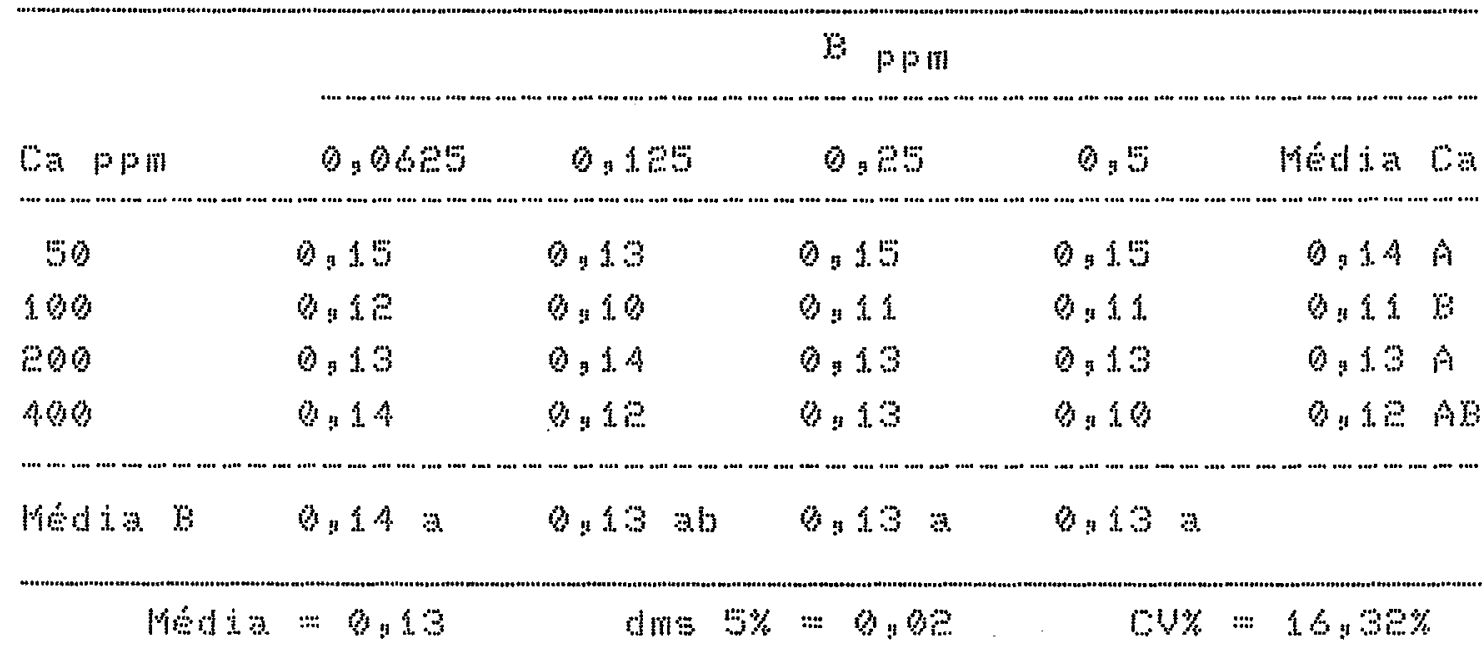

* Medias seguidas de letras maiúsculas na vertical e minúsculas na horizontal iguais nấ diferem entre si pelo teste de Tukey ao nivel de $5 \%$ de probabilidade.

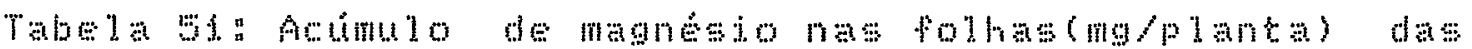

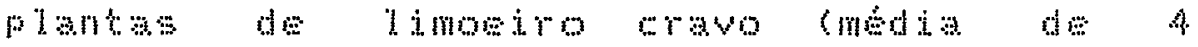
repetictses?

\begin{tabular}{|c|c|c|c|c|c|c|}
\hline & \multirow[b]{2}{*}{$p p m$} & \multicolumn{5}{|c|}{ E $P=m$} \\
\hline & & 0,0620 & $0, x=5$ & 0,25 & 0,5 & Médja \\
\hline 50 & & $4,1 \%$ & $3: 91$ & $4: 46$ & 4,41 & 4,208 \\
\hline 100 & & $4,6 \%$ & 4,37 & $4: 34$ & $3: 73$ & $4,32 \mathrm{p}$ \\
\hline 200 & & 10998 & $10,9 \%$ & 9.0 & 11906 & $10,71 \mathrm{~A}$ \\
\hline 400 & & $7_{y 14}$ & $9: 2$ & $10: 36$ & 8.99 & в \\
\hline
\end{tabular}

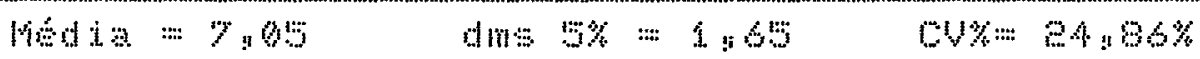

* Hedias seguidas de letras maúcuias na vertical e minúsulas na horizontal iguais nô̆ diferem entre si pelo teste de Tukey ao nivel de 5 to probabilidade. 


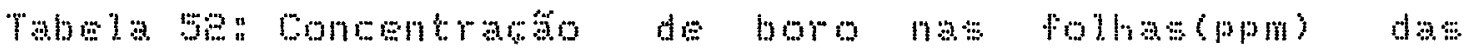
plantas de limouro cravo lnedia de a $\operatorname{reset}(60 \mathrm{~s})$

\begin{tabular}{|c|c|c|c|c|c|}
\hline \multirow[b]{2}{*}{ ca ppli } & \multicolumn{5}{|c|}{ B ppm } \\
\hline & 0,068 & $\theta, 185$ & 0,25 & 0.5 & Hétia oa \\
\hline 50 & 60,00 & $60: 150$ & 70.50 & $73: 50$ & 67,37 \\
\hline 1000 & 62 y & $54: 26$ & 54,65 & 79,79 & $57: 939$ \\
\hline 800 & $3 \%, 00$ & 10,100 & $44: 00$ & 59,00 & 40,120 \\
\hline 100 & $5 e, 00$ & $47: 09$ & $38: 78$ & 86473 & $49: 60 \%$ \\
\hline Modia $\mathrm{B}$ & $52,12 b$ & $34: 060$ & : 1. & $65_{10} 00 \%$ & \\
\hline
\end{tabular}

"hédias seguidas de letras maiúsculas na vertical e minúculas na horizontal iguais năo diferen entre si pelo teste de Tukes ao nivel de $5 \%$ de probabilidade.

Tabela ba folmulo de boro nas follas (ug/plantal das plantos do limosiro eravo smedia de 4 repetictos?.

\begin{tabular}{|c|c|c|c|c|c|}
\hline \multirow[b]{2}{*}{ Ca ppin } & \multicolumn{5}{|c|}{ g p pli } \\
\hline & 0,068 & 0,105 & $\theta, 2 ;$ & 0.5 & Media Ca \\
\hline 50 & $174: 43$ & 173,28 & 208,56 & 210,06 & 191.580 \\
\hline 100 & $191: 64$ & is: $18 \%$ & 6649 & «": 43 & 2449480 \\
\hline 800 & 328,76 & $373,: 54$ & $317: 46$ & 477,15 & $374,88 \mathrm{~A}$ \\
\hline sol & $28,8 \%$ & $388 \% 8$ & 308,4 & 47450 & $336,62 \mathrm{~A}$ \\
\hline \multicolumn{6}{|c|}{ (2) } \\
\hline Modia & $2 \%, 96 \mathrm{~b}$ & $2 \% 6096$ & 260,87 & 301,0 & \\
\hline
\end{tabular}

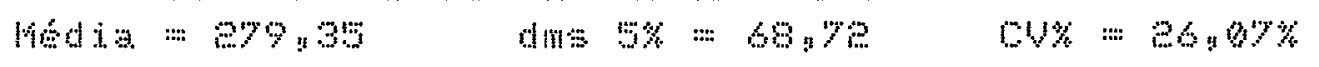

* Hédias sequidas de letras maícculas na vertical e minuscuias na horizontal iguais rä̀ diferem entre si pelo teste de Tukey an nivel de $5 \%$ de probabilidade. 


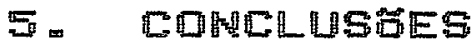

\author{
os resultados obtidos nas condiobs do

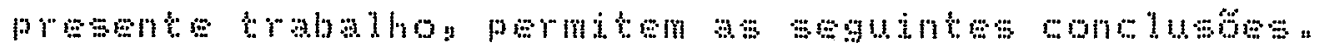

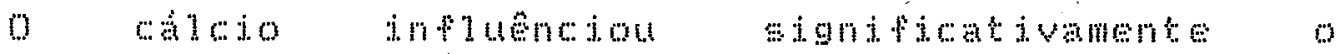 \\ desenvolvimento do sistoma radicular: o crosimento \\ da parte acrea e a composizo nineral de mudas do \\ limoeiro travon
}

Dos parknetros avaliados o boro apresentou resposta somente para o comprimento de radzes de limosiro wavon

Who houve efeito da incerato ca $x \quad 8$ sobre desenvolvimento do limomito mavo.

o melhor desenvolvimento de mudas do limoeiro eravon m soluga nutritiva ocorren no tratamento que continha eoppom de can 


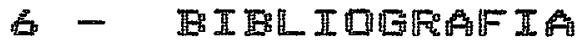

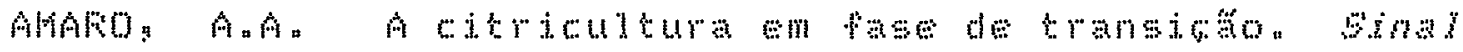

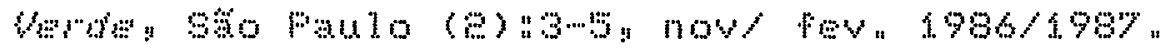

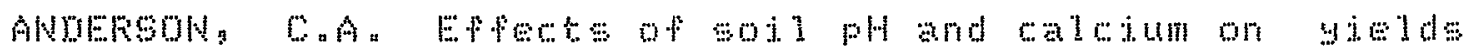
and fruit quatis of young "Ualeneis" oranges

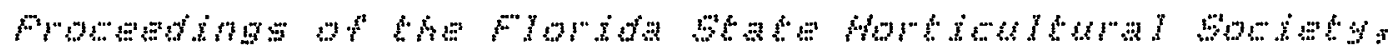

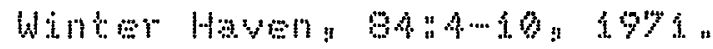

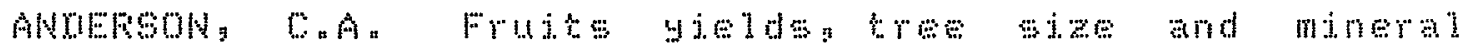
nutrition relationehipe in valentia onange trases 3

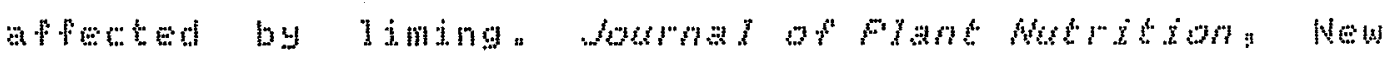

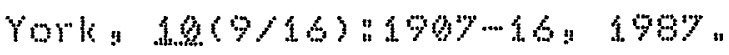

ASog Fus Tolertneda de plantines de portanjertos citricos a valos niveles de arbonato de calodo en el

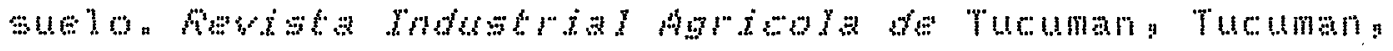
$5(1): 35,6 ; 5 \% 4$

ATKTNGoN, D. The distribution and efpectiveness of root

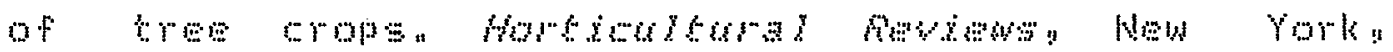


: :484-..9\%: 1960.

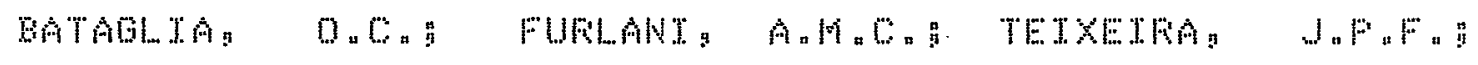

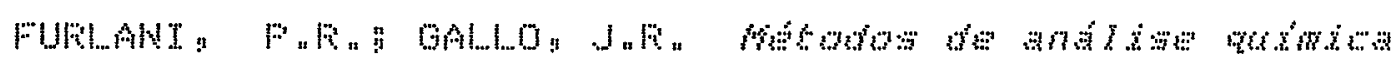

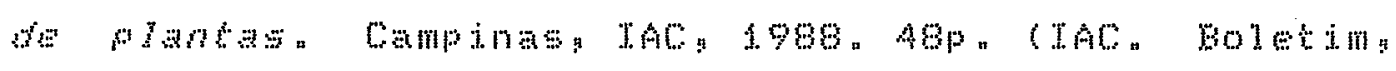
\%)

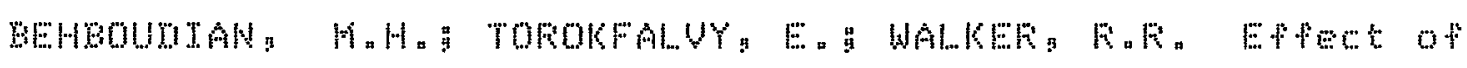
solinity on iontice contenty water relatons and as exthang paraneters in some citrus seionmootstok

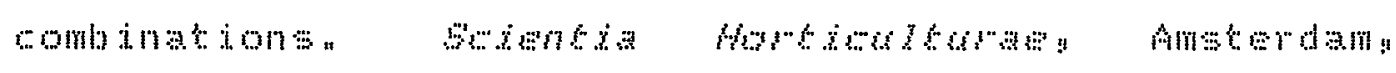

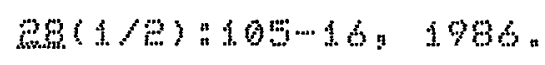

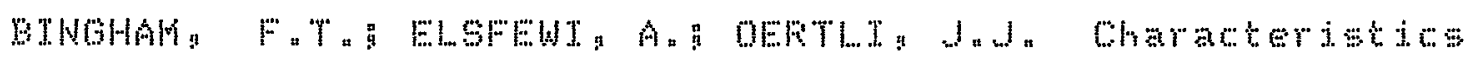
of boron aborption by oxejsed barles roots and

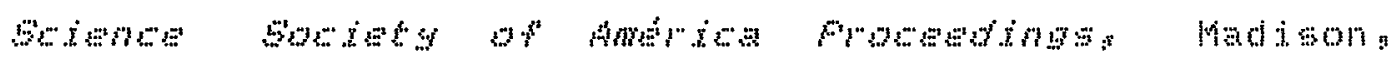

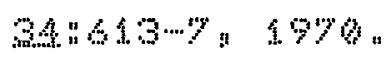

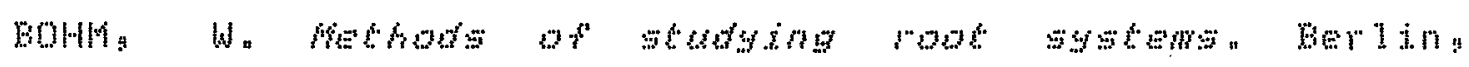

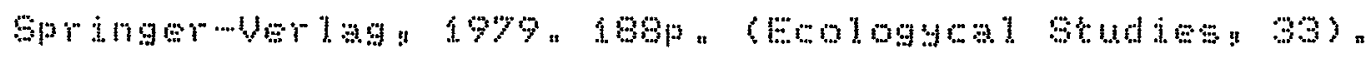

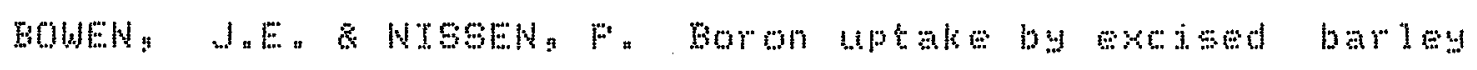

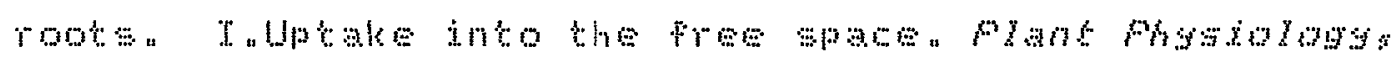

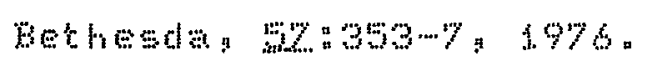

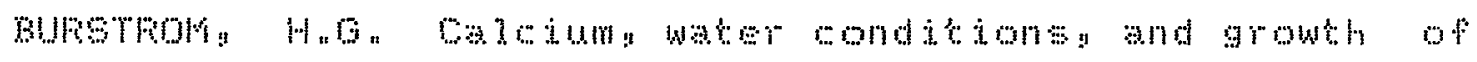

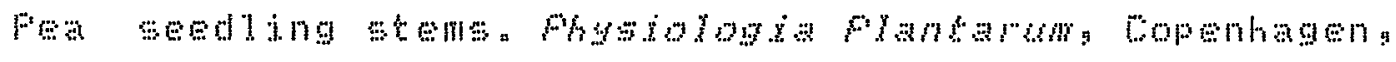


$12,007-219,1964$.

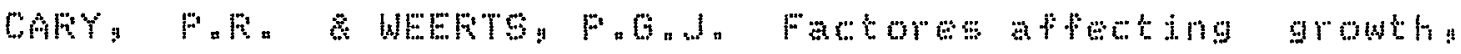
yield and prut compeition of washington Wavel and

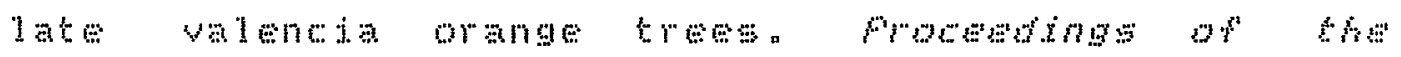

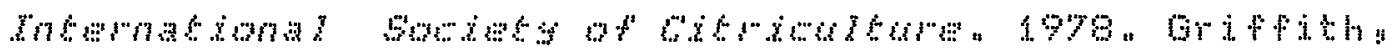
N. S.W. Australian $1980, p, 77-86$

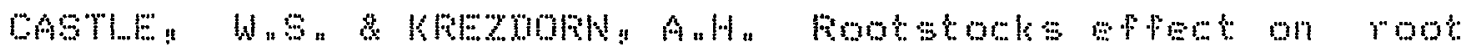
distribution and leat mineral content of "orlando"

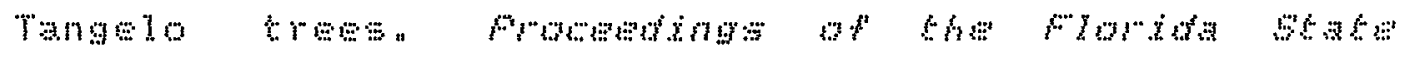

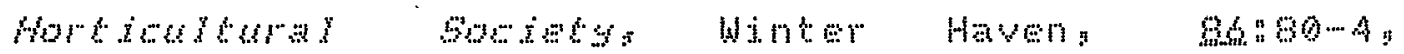
$1979 / 19 \% 4$ "

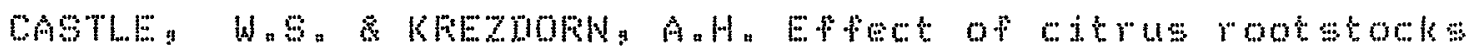
on root distribution and leat minoral content of "Orlando" tangelo trees. burna of the hamban

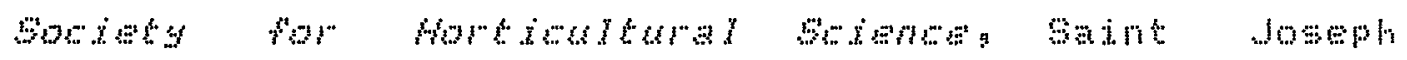
$100(1): 1 \cdots 4: 1975$

CHAFling, Hat The mineral nutrition of eitrus an

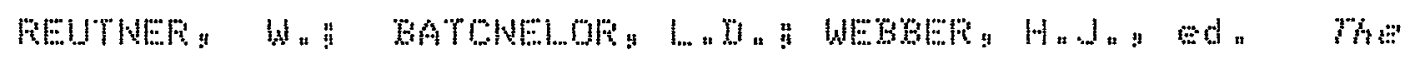

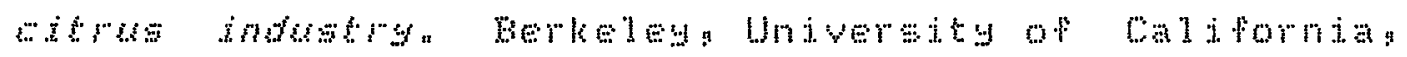

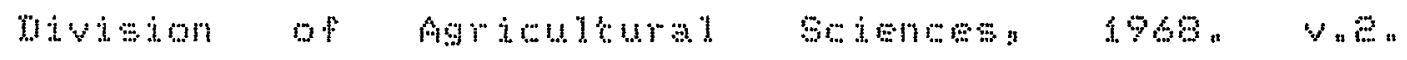
p.1.2....289. 


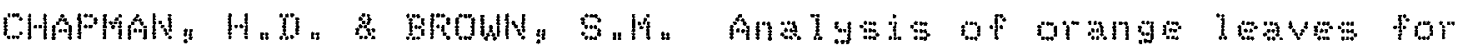
dianosing nutrient status with referenoe to potassium

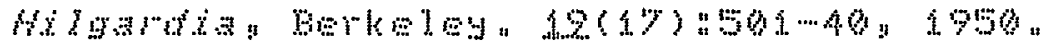

CHAUNHEY $F$ "M. $\&$ LONEFAOAN, J F" zino absomption by wheat

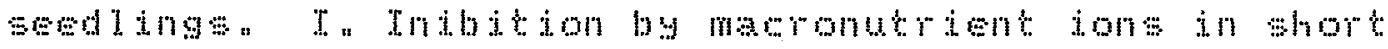
term studies and its relevmos to long torm zine

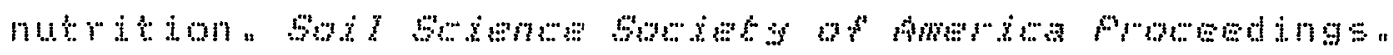

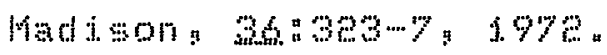

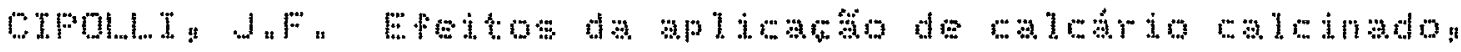
gesso o misturas caloúdomesso na cultura de citrosn I. Resultados preliminares Jabotional a 986 "49" Gonduato -.. Faculade de ciencias foraras e vetsinarias (UNES\%)

CHMKSON: MT" transocation of iron in bar les roots " Tracer and mitro

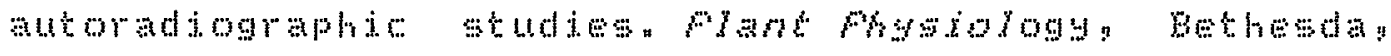

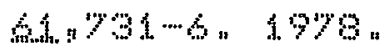

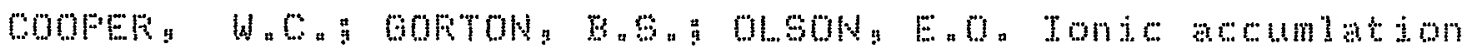
in wins as influenes by rootsock and seion and concentration of sat and boron in the subetrate fint

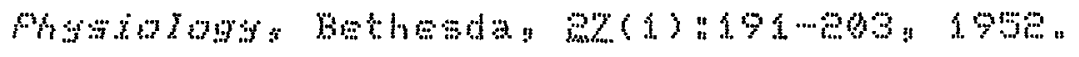




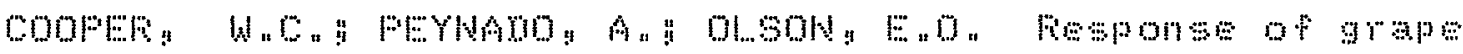
Prut on tor rootstocks to alosum addition to high sodium boron wontaminated and saline irrigation water.

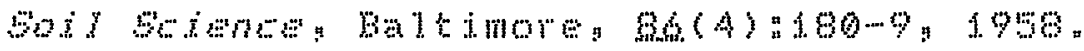

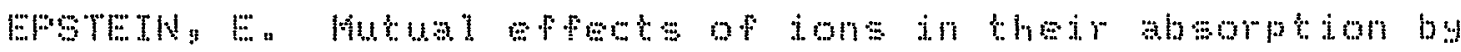

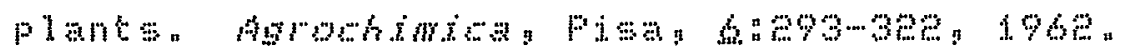

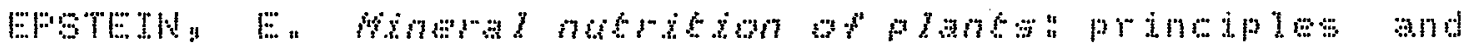

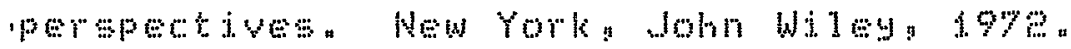

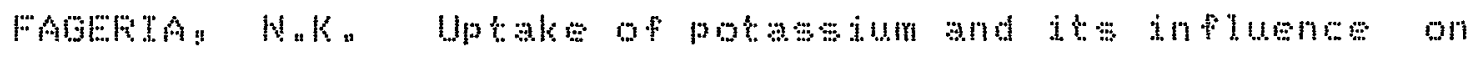

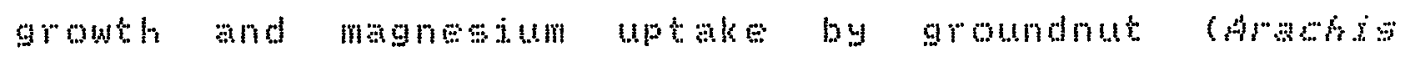

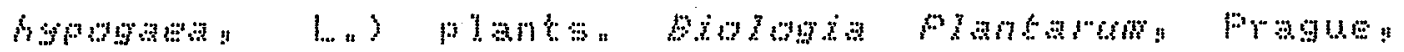

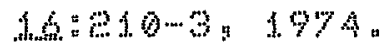

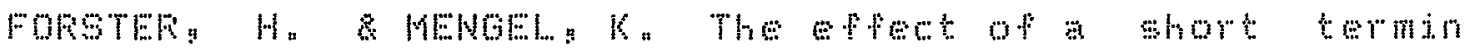
terruption an thek supply during the early stage on sield formation mineral content and soluble amino acid

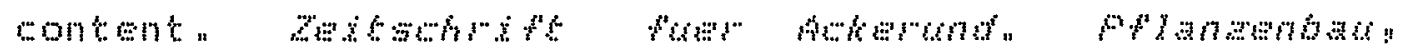
Hantorg: $190,203 \% 13,1969$.

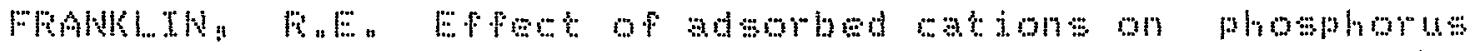

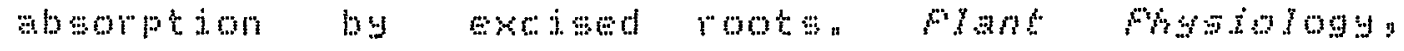

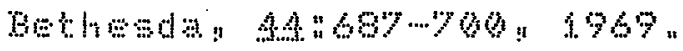




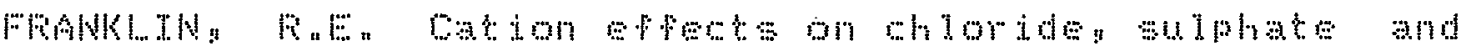

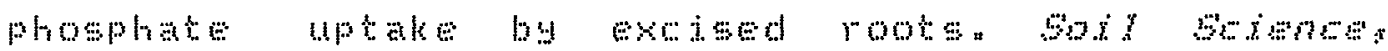

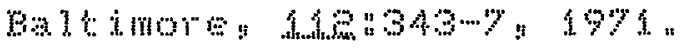

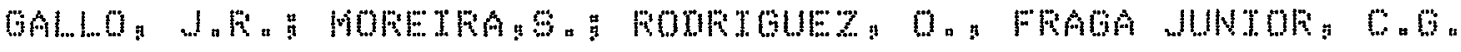
Influencia da varisdade e do portamenerto no

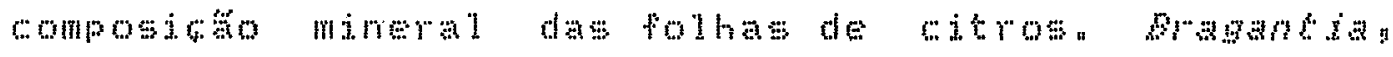
Camp inas: $19(20)$ " $307+18,1960$.

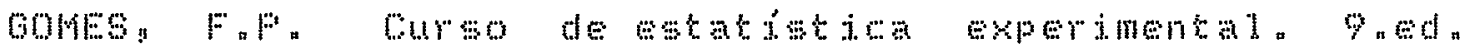

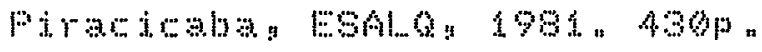

HAYNES: nas, Ton exchang properties of roots and ionie unteration within the roots apoplasm their role in

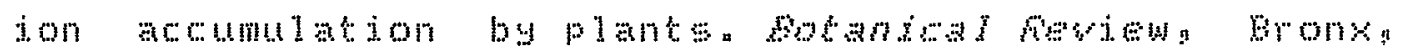
$34 \div 5 \% 9 \%, 1.960$

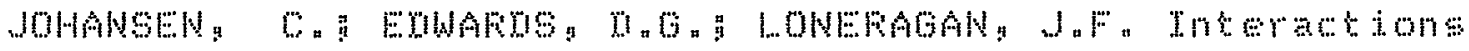
betwen potassum and alcium in their absontson by

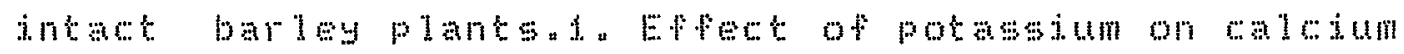

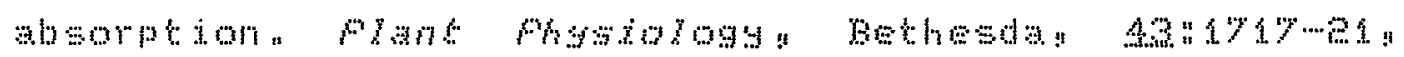
1960 :

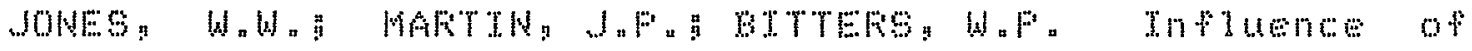
exomangate sodum and potassium in the sol on the arowh and compostion of young lemon trees on

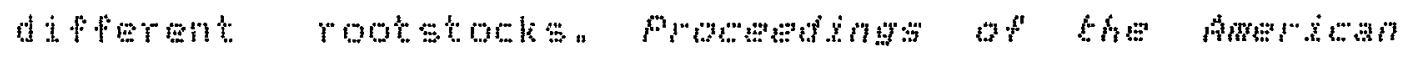




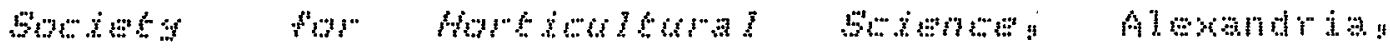
69:189-96, $19 \% \%$

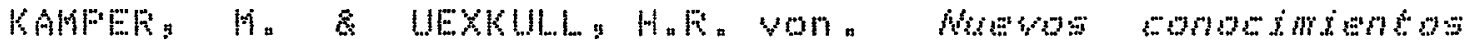

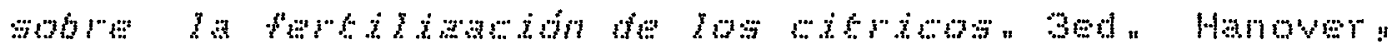
Uerlag oesselechat fur Ackerbaug $1966,104 \%$

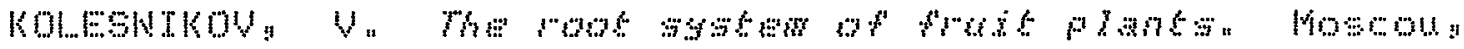
Minublisher: 19\% a $66 \%$.

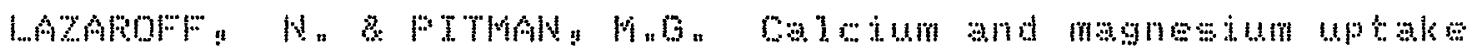

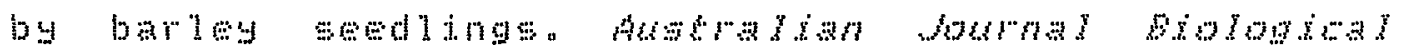

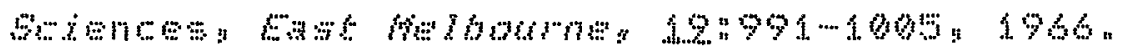

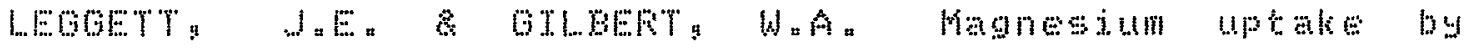

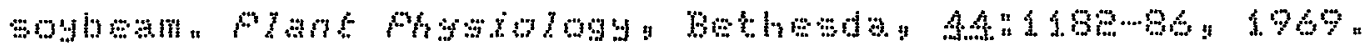

LWMA LaAn de Intuencia de diferentes portankentose snxertos na varaso da monentrato do maconutrientes

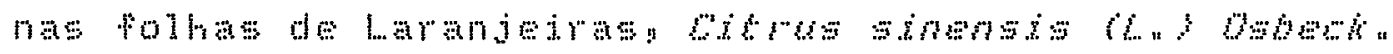

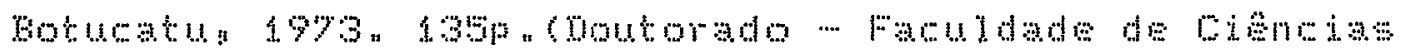
Medicas ojologicas de Boturatur.

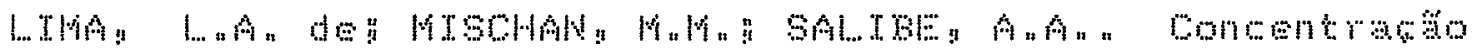

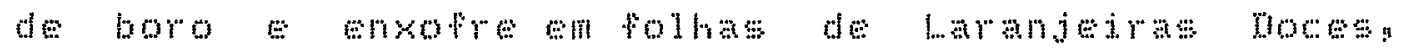
determindas por diperentes portamenertos newsto

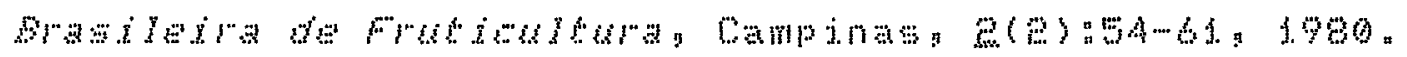




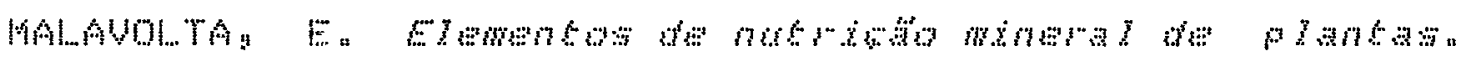

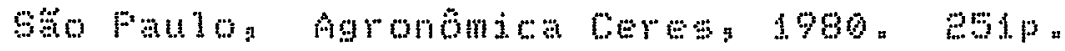

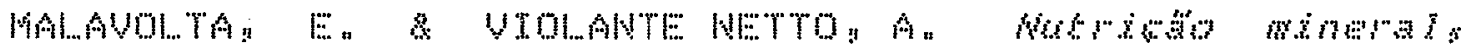
Associngo Brasileira para mesuisa da Fotassa e do Fostrogo, 1989, 153\%.

MALAUOLTA E. UTTTI 0 "

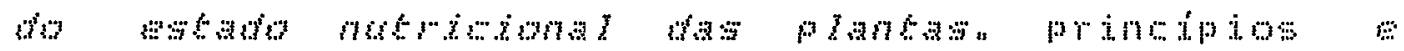
aplicacoses Firacicabag Associacto Brasileira para

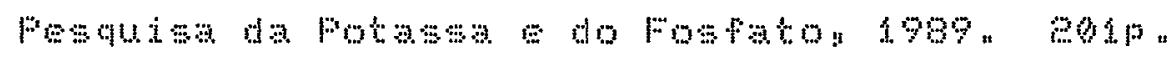

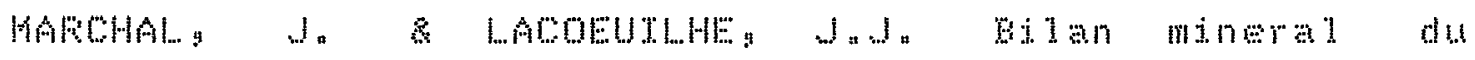

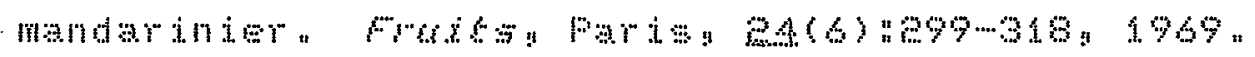

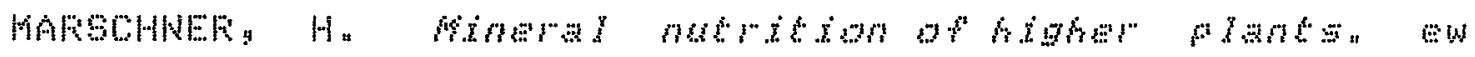

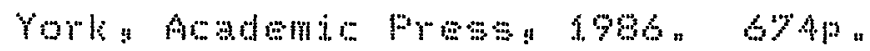

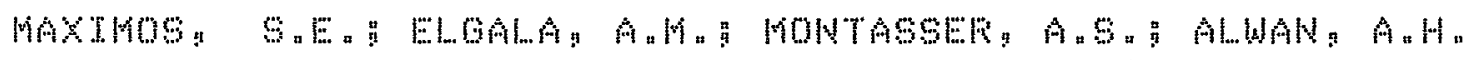

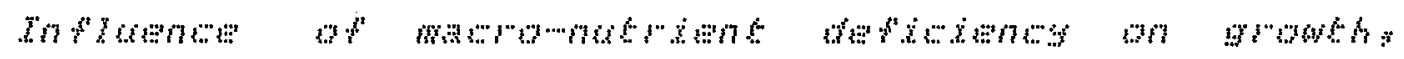

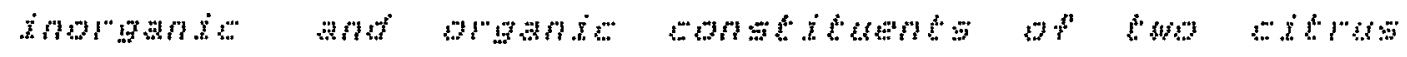

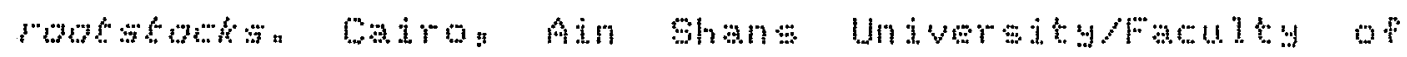

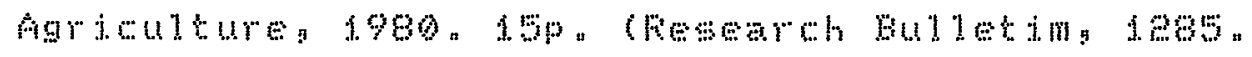

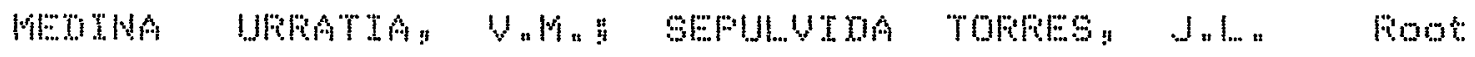
distribution of six mexican lime rootstoms. 


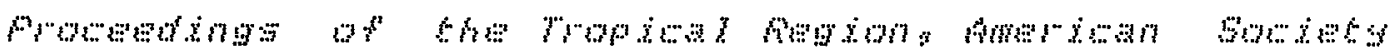

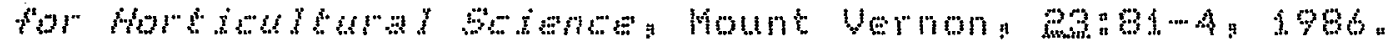

METINA URRATA Analusis of the root distribution of eigh rootstows

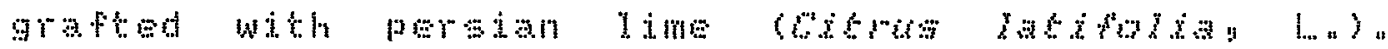

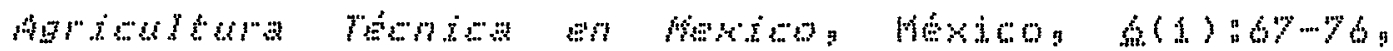
$198 \%$

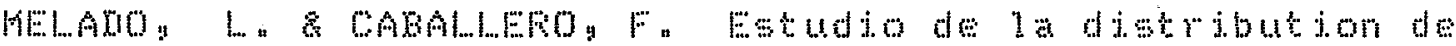

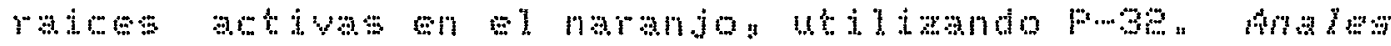

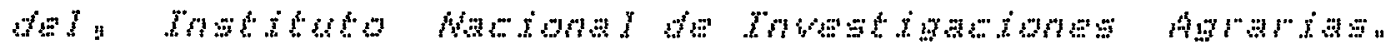

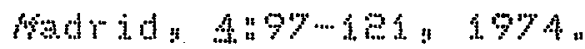

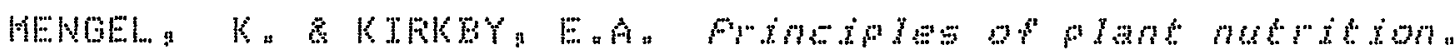

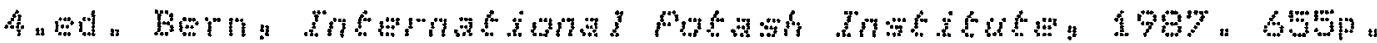

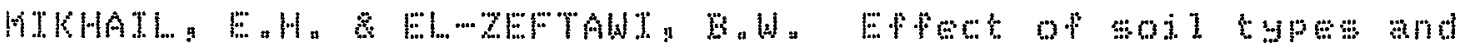
rootstows on root distribution and leat compostion of

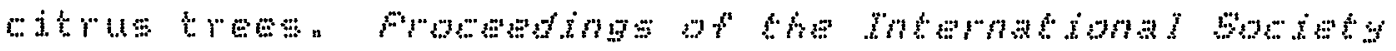

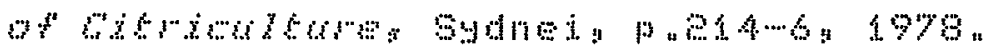

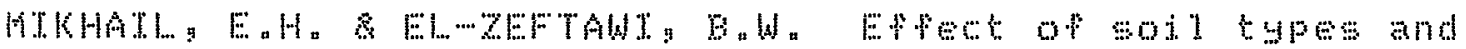
rootstocks on root distribution shemisal composition

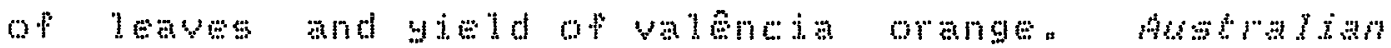

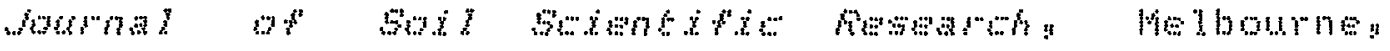
$12(0): 39549 ; 197 \%$ 


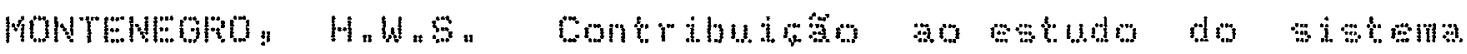

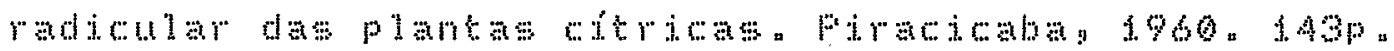

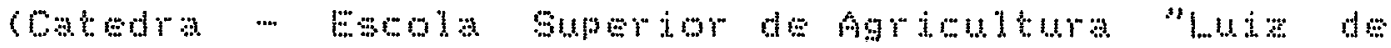
Queiroz/Us:?

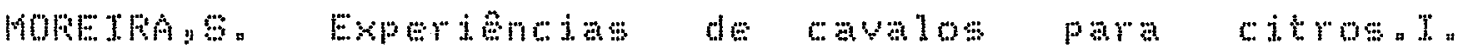

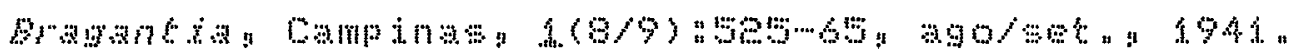

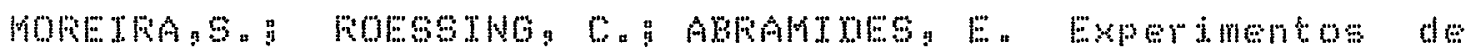

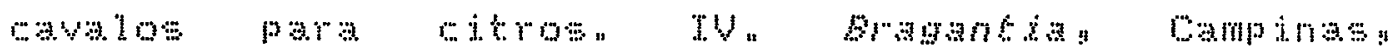

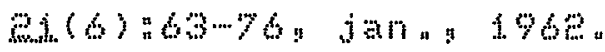

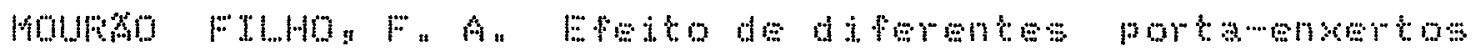
no vigor e produtiviade das plantas e nas

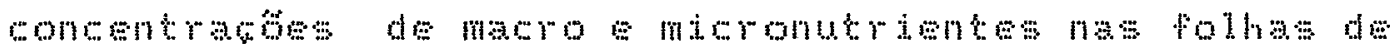

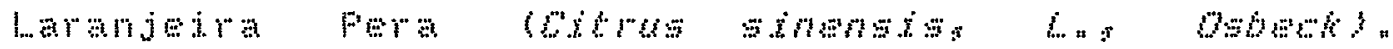

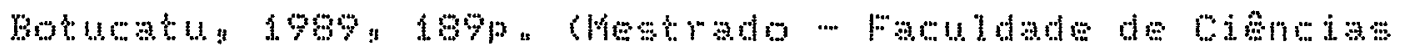

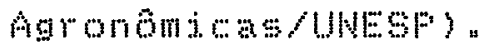

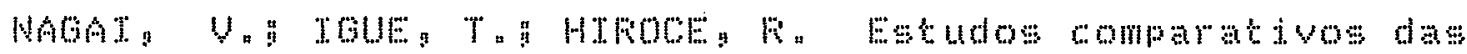
relases ontre os nutrientes dosador nas bonas de

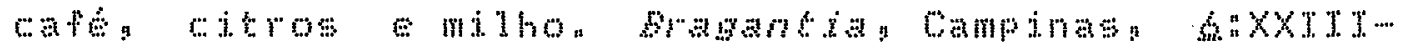

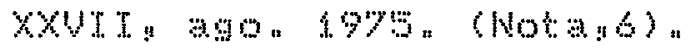

NEWMAN, Ex. A mothod of wstmating the total length of

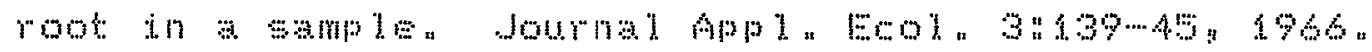




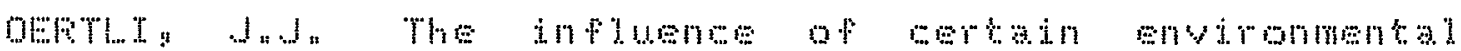
conditions on water and nutrient uptake and nutrition distribution in barley serdlings whth spoial reference

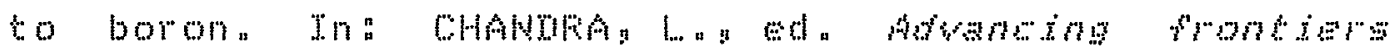

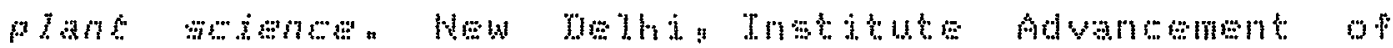
scticnce and oulture, $1963, \quad p, 65-8 \%$

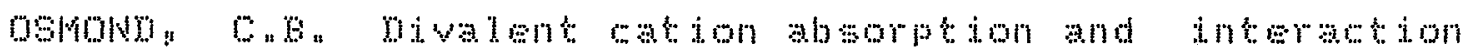

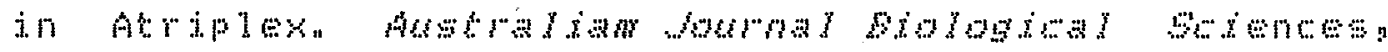
East Melbourne

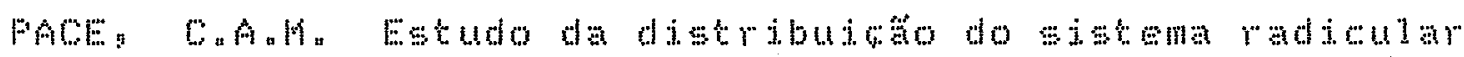
de portamentortos citricos en solos podzolizados fio

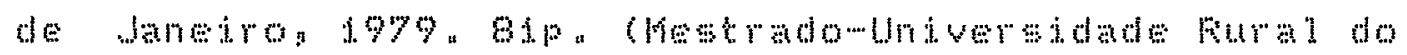
Fio de Janeiroln

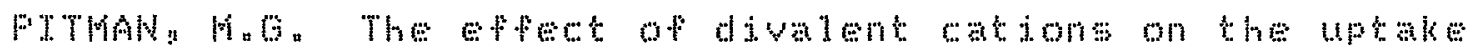

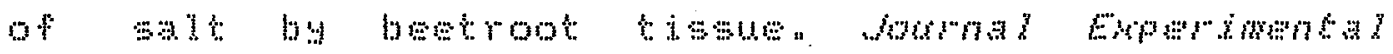

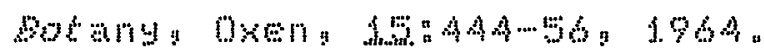

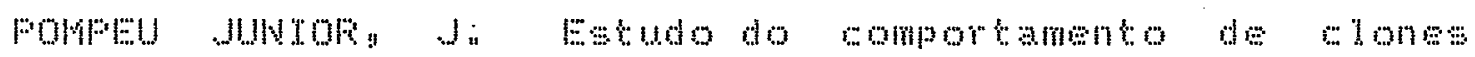

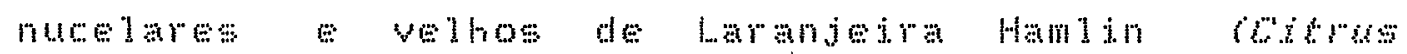

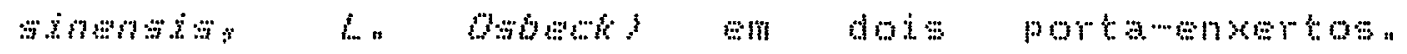
Firmodoma 197en 77p

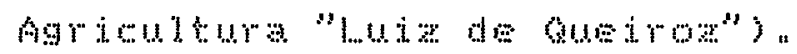




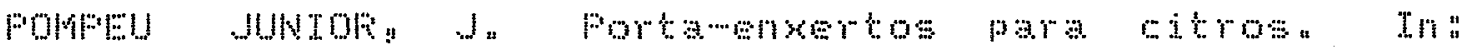

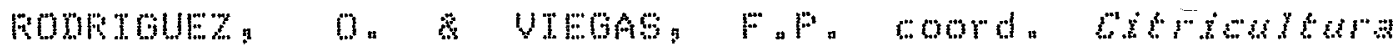

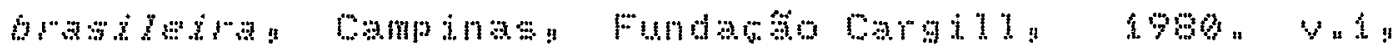

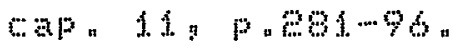

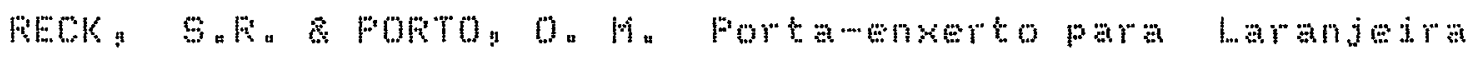

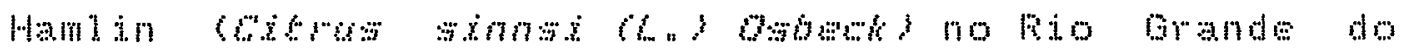

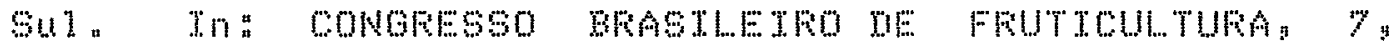

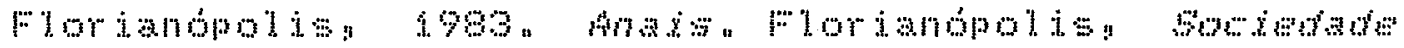

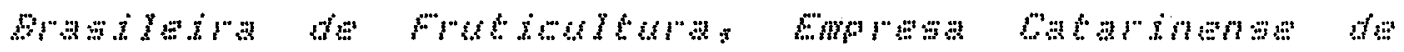

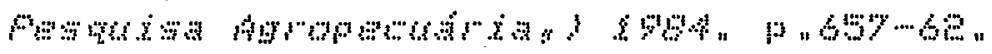

RETTz Hol a sTriEs; Wo. Fertilization of high

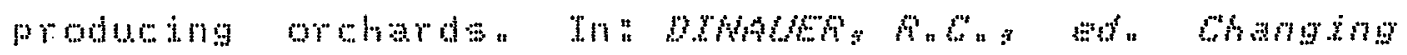

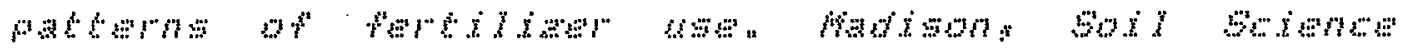
क्w.

RTCHADOS "

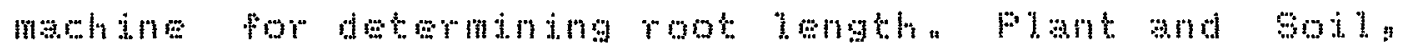
The Wague g

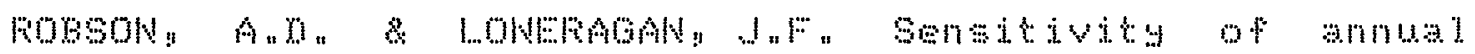
Wedrago species to manganese toxicity as affected by

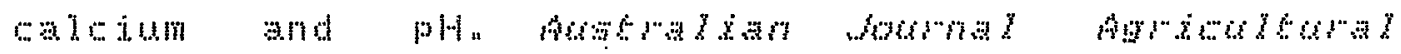

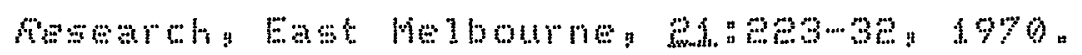




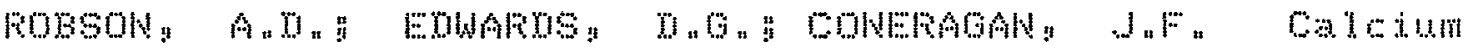
Stimulation of phospats absompton by annul legumes"

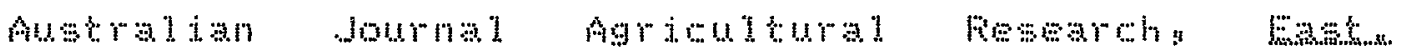

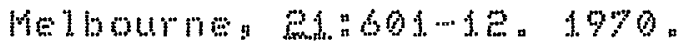

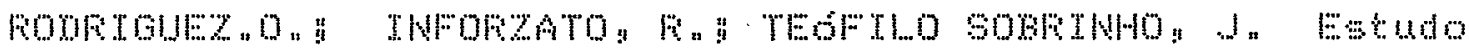

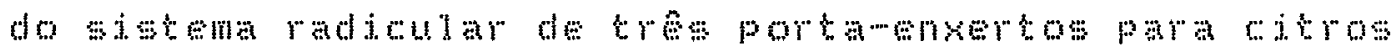

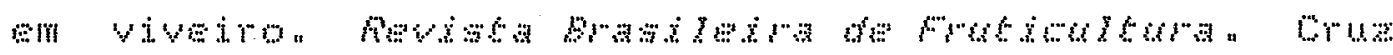
das A1ma: $4(1)$ a $23-30,1978$.

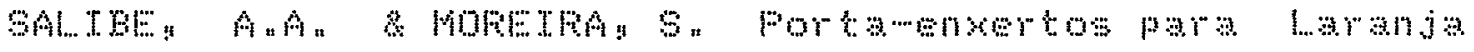

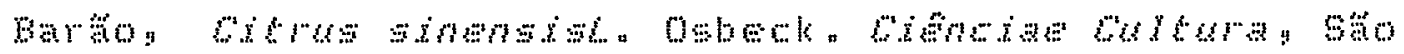

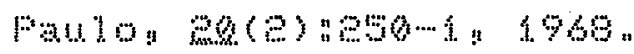

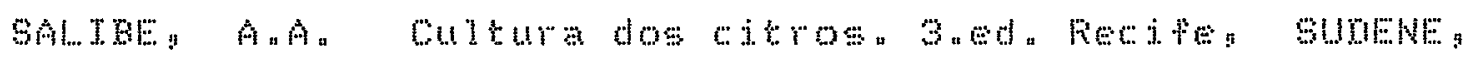
Un versida Fedral Rural do Fernambon $19 \%$ " $180 \%$

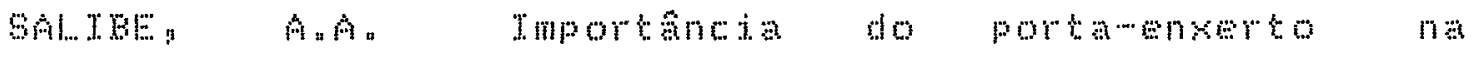

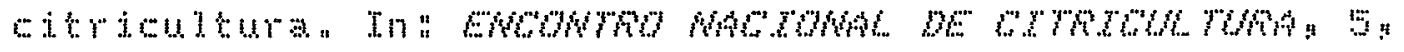

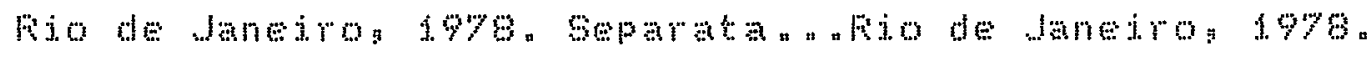

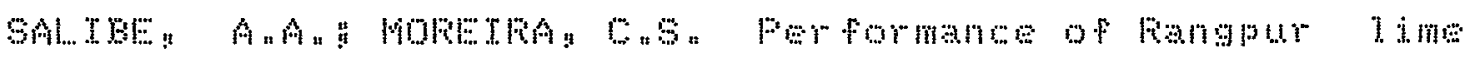

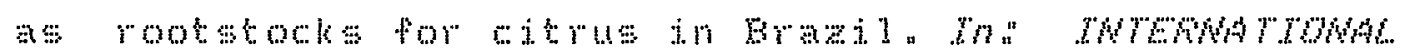
-

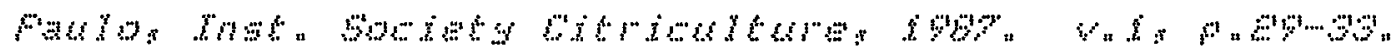

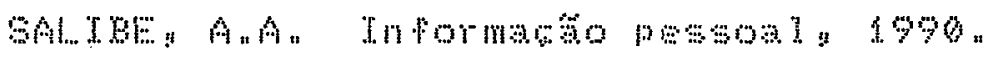




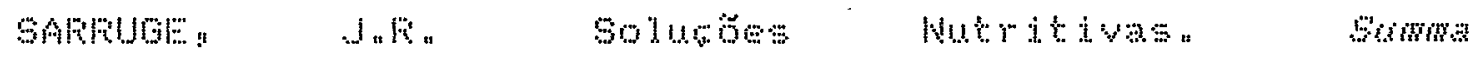

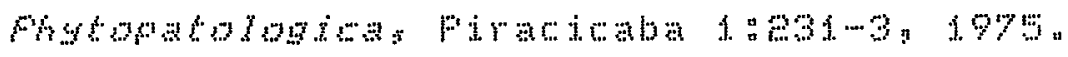

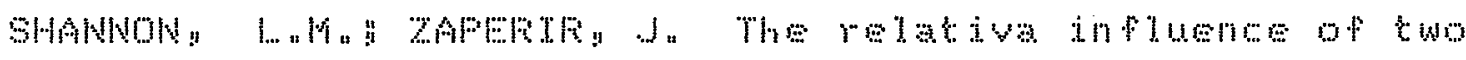
citrus rootstocks specise upon plant growt and upon

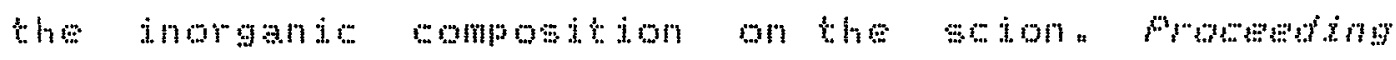

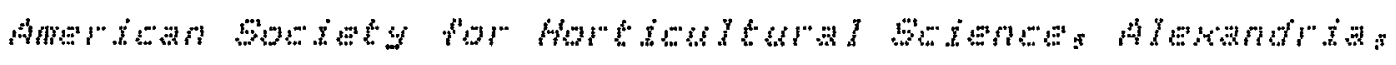

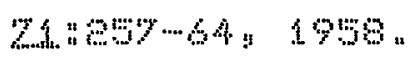

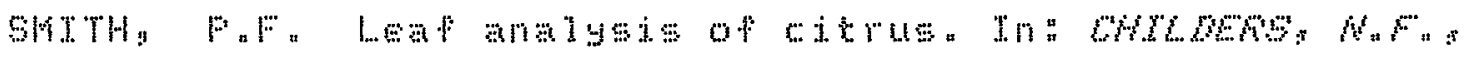

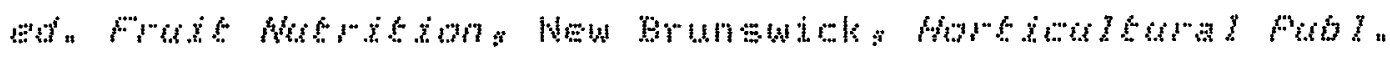
Awtas: $1969,179 \%$

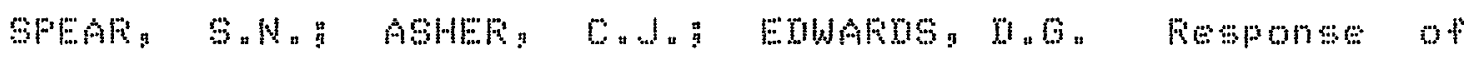

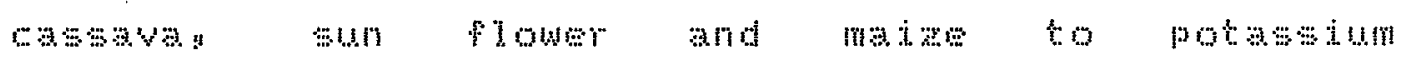
conoutration in solution. $x$. Orowth and plant

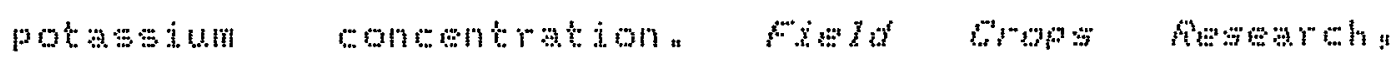
Anstrerdang $1: 347+61$ a $1978 \mathrm{a}$

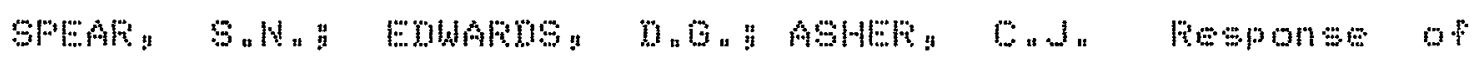
cassavan sunflower and maje to potasion concentration

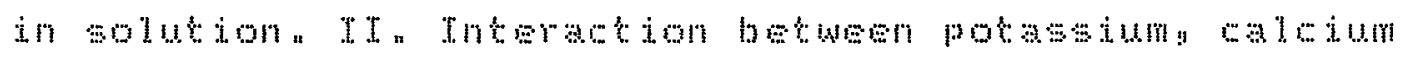

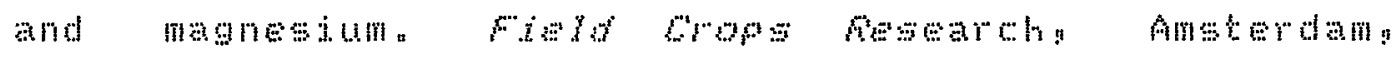

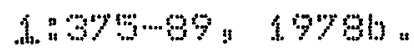

D

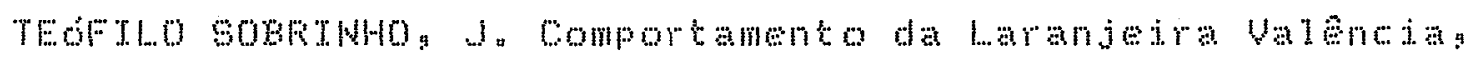

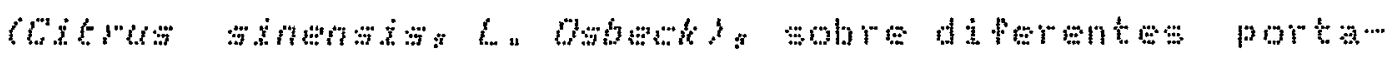




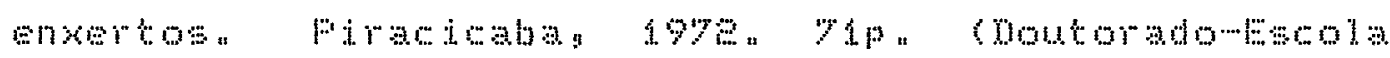

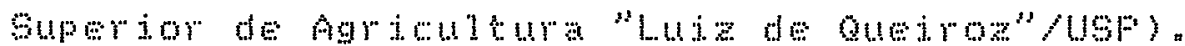

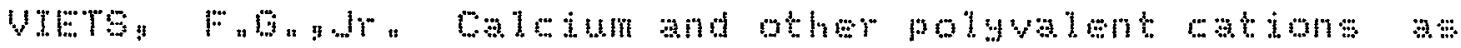
aceserator of ion accumulaton by excised barley

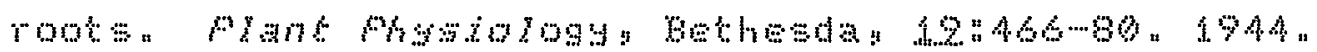

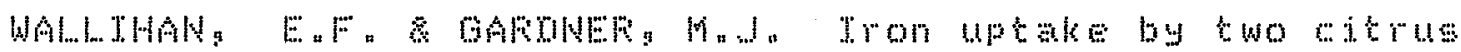
rootstoese wpeces in relation to soll moisure and

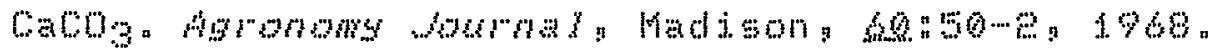

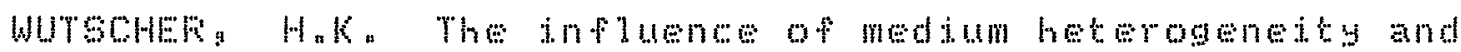
three rootstows on growthand nutrient levels of greenhousewown "Ualeneia" orange trees bounar of

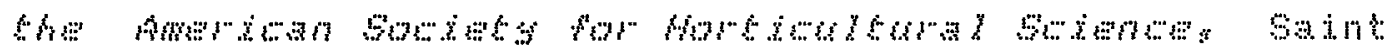

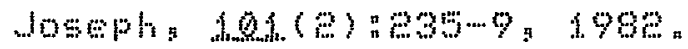

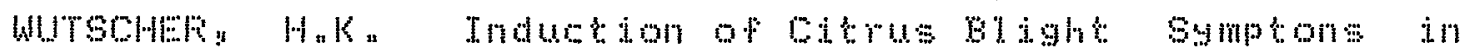
3-yermold "Hamlim" treses in solution culture.

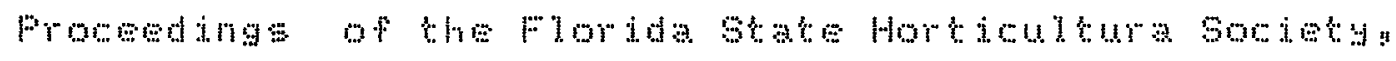

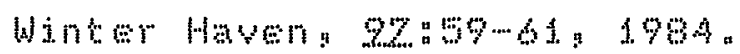

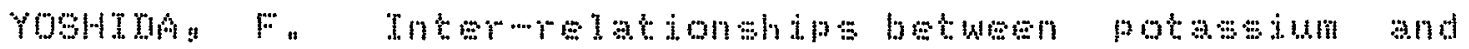

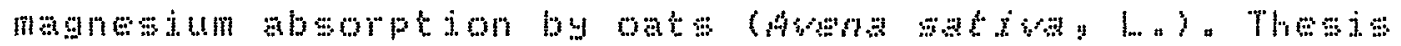
state Agric " wniv wagentngena 2966 . 